\title{
Stochastic Navier-Stokes Equations Driven by Lévy Noise in Unbounded 3D Domains
}

\section{Elżbieta Motyl}

Received: 1 February 2012 / Accepted: 11 July 2012 / Published online: 24 August 2012

(C) The Author(s) 2012. This article is published with open access at Springerlink.com

\begin{abstract}
Martingale solutions of the stochastic Navier-Stokes equations in 2D and 3D possibly unbounded domains, driven by the Lévy noise consisting of the compensated time homogeneous Poisson random measure and the Wiener process are considered. Using the classical Faedo-Galerkin approximation and the compactness method we prove existence of a martingale solution. We prove also the compactness and tightness criteria in a certain space contained in some spaces of càdlàg functions, weakly càdlàg functions and some Fréchet spaces. Moreover, we use a version of the Skorokhod Embedding Theorem for nonmetric spaces.
\end{abstract}

Keywords Stochastic Navier-Stokes equations • Martingale solution • Poisson random measure $\cdot$ Compactness method

Mathematics Subject Classifications (2010) $35 \mathrm{Q} 30 \cdot 60 \mathrm{H} 15 \cdot 76 \mathrm{M} 35$

\section{Introduction}

Let $\mathscr{O} \subset \mathbb{R}^{d}$ be an open connected possibly unbounded subset with smooth boundary $\partial \mathscr{O}$, where $d=2,3$. We will consider the Navier-Stokes equations

$$
\begin{aligned}
d u(t)= & {[\Delta u-(u \cdot \nabla) u+\nabla p+f(t)] d t+\int_{Y} F(t, u) \tilde{\eta}(d t, d y) } \\
& +G(t, u(t)) d W(t), \quad t \in[0, T],
\end{aligned}
$$

in $\mathscr{O}$, with the incompressibility condition

$$
\operatorname{div} u=0,
$$

E. Motyl $(\varangle)$

Department of Mathematics and Computer Sciences, Lodz University, Banacha 22,

91-238 Łódź, Poland

e-mail: emotyl@math.uni.lodz.pl 
the initial condition

$$
u(0)=u_{0},
$$

and with the homogeneous boundary condition $u_{\mid \partial \mathscr{O}}=0$. In this problem $u=$ $u(t, x)=\left(u_{1}(t, x), \ldots u_{d}(t, x)\right)$ and $p=p(t, x)$ represent the velocity and the pressure of the fluid, respectively. Furthermore, $f$ stands for the deterministic external forces. The terms $\int_{Y} F(t, u) \tilde{\eta}(d t, d y)$, where $\tilde{\eta}$ is a compensated time homogeneous Poisson random measure on a certain measurable space $(Y, \mathscr{Y})$, and $G(t, u(t)) d W(t)$, where $W$ is a cylindrical Wiener process on some separable Hilbert space $Y_{W}$, stand for the random forces.

The problem (1-3) can be written as the following stochastic evolution equation

$$
\begin{aligned}
d u(t) & +\mathscr{A} u(t) d t+B(u(t)) d t=f(t) d t+\int_{Y} F\left(t, u\left(t^{-}\right) ; y\right) \tilde{\eta}(d t, d y) \\
& +G(t, u(t)) d W(t) \quad t \in[0, T], \\
u(0) \quad & =u_{0} .
\end{aligned}
$$

We will prove the existence of a martingale solution of the problem (1-3) understood as a system $(\Omega, \mathscr{F}, \mathbb{P}, \mathbb{F}, \eta, W, u)$, where $(\Omega, \mathscr{F}, \mathbb{P}, \mathbb{F})$ is a filtered probability space, $\eta$ is a time homogeneous Poisson random measure, $W$ is a cylindrical Wiener process and $u=\left(u_{t}\right)_{t \in[0, T]}$ is a stochastic process with trajectories in the space $\mathbb{D}\left([0, T], H_{w}\right) \cap$ $L^{2}(0, T ; V)$ and satisfying appropriate integral equality, see Definition 3 in Section 4.2. Here, $V$ and $H$ denote the closures in $H^{1}\left(\mathscr{O}, \mathbb{R}^{d}\right)$ and $L^{2}\left(\mathscr{O}, \mathbb{R}^{d}\right)$, respectively of the space $\mathscr{V}$ of the divergence-free $\mathbb{R}^{d}$ valued vector fields of class $\mathscr{C}^{\infty}$ with compact supports contained in $\mathscr{O}$. The symbol $\mathbb{D}\left([0, T], H_{w}\right)$ stands for the space of $H$ valued weakly càdlàg functions.

To construct this solution we use the classical Faedo-Galerkin method, i.e.,

$$
\begin{aligned}
d u_{n}(t)= & -\left[P^{n} \mathscr{A} u_{n}(t)+B_{n}\left(u_{n}(t)\right)-P_{n} f(t)\right] d t \\
& +\int_{Y} P_{n} F\left(t, u_{n}\left(t^{-}\right), y\right) \tilde{\eta}(d t, d y)+P_{n} G(t, u(t)) d W(t), t \in[0, T], \\
u_{n}(0)= & P_{n} u_{0} .
\end{aligned}
$$

The solutions $u_{n}$ to the Galerkin scheme generate a sequence of laws $\left\{\mathscr{L}\left(u_{n}\right)\right.$, $n \in \mathbb{N}\}$ on appropriate functional spaces. To prove that this sequence of probability measures is weakly compact we need appropriate tightness criteria.

We concentrate first on the compactness and tightness criteria. If the domain $\mathscr{O}$ is unbounded, then the embedding $V \subset H$ is not compact. However using Lemma 2.5 in [16], see Appendix C, we can find a separable Hilbert space $U$ such that $U \subset V$, the embedding being dense and compact.

We consider the intersection

$$
\mathscr{Z}_{q}:=L_{w}^{q}(0, T ; V) \cap L^{q}\left(0, T ; H_{l o c}\right) \cap \mathbb{D}\left([0, T] ; U^{\prime}\right) \cap \mathbb{D}\left([0, T], H_{w}\right),
$$

where $q \in(1, \infty)$. (The letter $w$ indicates the weak topology.) By $\mathbb{D}\left([0, T] ; U^{\prime}\right)$ we denote the space of $U^{\prime}$-valued càdlàg functions equipped with the Skorokhod topology and $L^{q}\left(0, T ; H_{\mathrm{loc}}\right)$ stands for the Fréchet space defined by Eq. 24, see Section 3.2. 
Using the compactness criterion in the space of càdlàg functions, we prove that a set $\mathscr{K}$ is relatively compact in $\mathscr{L}_{q}$ if the following three conditions hold

(a) for all $u \in \mathscr{K}$ and all $t \in[0, T], u(t) \in H$ and $\sup _{u \in \mathscr{K}} \sup _{s \in[0, T]}|u(s)|_{H}<\infty$,

(b) $\sup _{u \in \mathscr{K}} \int_{0}^{T}\|u(s)\|_{V}^{q} d s<\infty$, i.e. $\mathscr{K}$ is bounded in $L^{q}(0, T ; V)$,

(c) $\lim _{\delta \rightarrow 0} \sup _{u \in \mathscr{K}} w_{[0, T], U^{\prime}}(u ; \delta)=0$.

Here $w_{[0, T], U^{\prime}}(u ; \delta)$ stands for the modulus of the function $u:[0, T] \rightarrow U^{\prime}$. The above result is a straightforward generalization of the compactness results of [9] and [25]. In the paper [25] the analogous result is proved in the case when the embedding $V \subset H$ is dense and compact (in the Banach space setting). In [9] the embedding $V \subset H$ is only dense and continuous. However, instead of the spaces of càdlàg functions, appropriate spaces of continuous functions are used. The present paper generalizes both [9] and [25] in the sense that the embedding $V \subset H$ is dense and continuous and appropriate spaces of càdlàg functions are considered, i.e. $\mathbb{D}\left([0, T] ; U^{\prime}\right)$ and $\mathbb{D}\left([0, T], H_{w}\right)$. This approach were strongly inspired by the results due to Métivier and Viot, especially the choice of the spaces $\mathbb{D}\left([0, T] ; U^{\prime}\right)$ and $\mathbb{D}\left([0, T], H_{w}\right)$, see $[23]$ and [22]. It is also closely related to the result due to Mikulevicius and Rozovskii [24] and to the classical Dubinsky compactness criterion, [28]. However, both in [28] and [24], the spaces of continuous functions are used.

Using the above deterministic compactness criterion and the Aldous condition in the form given by Joffe and Métivier [19], see also [22], we obtain the corresponding tightness criterion for the laws on the space $\mathscr{Z}_{q}$, see Corollary 1.

We will prove that the set of probability measures induced by the Galerkin solutions is tight on the space $\mathscr{Z}$, where

$$
\mathscr{Z}:=L_{w}^{2}(0, T ; V) \cap L^{2}\left(0, T ; H_{\mathrm{loc}}\right) \cap \mathbb{D}\left([0, T] ; U^{\prime}\right) \cap \mathbb{D}\left([0, T] ; H_{w}\right),
$$

which is not metrizable. Further construction a martingale solutions is based on the Skorokhod Embedding Theorem in nonmetric spaces. In fact, we use the result proved in [25] and following easily from the Jakubowski's version of the Skorokhod Theorem [18] and the version of the Skorokhod Theorem due to Brzeźniak and Hausenblas [6], see Appendix B. This will allow us to construct a stochastic process $\bar{u}$ with trajectories in the space $\mathscr{Z}$, a time homogeneous Poisson random measure $\bar{\eta}$ and a cylindrical Wiener proces $\bar{W}$ defined on some filtered probability space $(\bar{\Omega}, \overline{\mathscr{F}}, \overline{\mathbb{P}}, \overline{\mathbb{F}})$ such that the system $(\bar{\Omega}, \overline{\mathscr{F}}, \overline{\mathbb{P}}, \overline{\mathbb{F}}, \bar{\eta}, \bar{W}, \bar{u})$ is a martingale solution of the problem (1-3). In fact, $\bar{u}$ is a process with trajectiories in the space $\mathscr{Z}$. In particular, the trajectories of $\bar{u}$ are weakly càdlàg if $\bar{u}$ is considered as a $H$-valued process and càdlàg in the bigger space $U^{\prime}$.

The Navier-Stokes equations driven by the compensated Poisson random measure in the 3D bounded domains were studied in Dong and Zhai [15]. The authors consider the martingale problem associated to the Navier-Stokes equations, i.e. a solution is defined to be a probability measure satisfying appropriate conditions, see Definition 3.1 in [15]. The 2D Navier-Stokes equations were considered in [13, 14] and [29]. In the present paper, using a different approach we generalize the existence resuls to the case of unbounded 2D and 3D domains. Moreover, we consider more general noise term.

Stochastic Navier-Stokes equations in unbounded 2D and 3D domains were usually considered with the Gaussian noise term, see e.g. [8, 11, 12] and [9]. Martingale 
solutions of the stochastic Navier-Stokes equations driven by white noise in the whole space $\mathbb{R}^{d},(d \geq 2)$, are investigated in [24].

The present paper is organized as follows. In Section 2 we recall basic definitions and properties of the spaces and operators appearing in the Navier-Stokes equations. Section 3 is devoted to the compactness and tightness results. Some auxilliary results about the Aldous condition and tightness are contained in Appendix A. Precise statement of the Navier-Stokes problem driven by Lévy noise is contained in Section 4.2. The main Theorem about existence of a martingale solution of the problem (13 ) is proved in Section 5. Some versions the Skorokhod Embedding Theorems are recalled in Appendix B. In Appendix C we recall Lemma 2.5 in [16] together with the proof.

\section{Functional Setting}

\subsection{Basic Definitions}

Let $\mathscr{O} \subset \mathbb{R}^{d}$ be an open connected subset with smooth boundary $\partial \mathscr{O}, d=2,3$. Let

$$
\begin{aligned}
\mathscr{V} & :=\left\{u \in \mathscr{C}_{c}^{\infty}\left(\mathscr{O}, \mathbb{R}^{d}\right): \operatorname{div} u=0\right\}, \\
H & :=\text { the closure of } \mathscr{V} \text { in } L^{2}\left(\mathscr{O}, \mathbb{R}^{d}\right), \\
V & :=\text { the closure of } \mathscr{V} \text { in } H^{1}\left(\mathscr{O}, \mathbb{R}^{d}\right) .
\end{aligned}
$$

In the space $H$ we consider the scalar product and the norm inherited from $L^{2}\left(\mathscr{O}, \mathbb{R}^{d}\right)$ and denote them by $(\cdot \mid \cdot)_{H}$ and $|\cdot|_{H}$, respectively, i.e.

$$
(u \mid v)_{H}:=(u \mid v)_{L^{2}}, \quad|u|_{H}:=\|u\|_{L^{2}}, \quad u, v \in H .
$$

In the space $V$ we consider the scalar product inherited from the Sobolev space $H^{1}\left(\mathscr{O}, \mathbb{R}^{d}\right)$, i.e.

$$
(u \mid v)_{V}:=(u \mid v)_{L^{2}}+((u \mid v))
$$

where

$$
((u \mid v)):=(\nabla u \mid \nabla v)_{L^{2}}, \quad u, v \in V .
$$

and the norm

$$
\|u\|_{V}^{2}:=|u|_{H}^{2}+\|u\|^{2}
$$

where $\|u\|^{2}:=\|\nabla u\|_{L^{2}}^{2}$.

2.2 The Form $b$

Let us consider the following three-linear form, see Temam [27],

$$
b(u, w, v)=\int_{\mathscr{O}}(u \cdot \nabla w) v d x .
$$


We will recall those fundamental properties of the form $b$ that are valid both in bounded and unbounded domains. By the Sobolev embedding Theorem, see [1], and the Hólder inequality, we obtain the following estimates

$$
|b(u, w, v)| \leq c\|u\|_{V}\|w\|_{V}\|v\|_{V}, \quad u, w, v \in V
$$

for some positive constant $c$. Thus the form $b$ is continuous on $V$, see also [27]. Moreover, if we define a bilinear map $B$ by $B(u, w):=b(u, w, \cdot)$, then by inequality (9) we infer that $B(u, w) \in V^{\prime}$ for all $u, w \in V$ and that the following inequality holds

$$
|B(u, w)|_{V^{\prime}} \leq c\|u\|_{V}\|w\|_{V}, \quad u, w \in V .
$$

Moreover, the mapping $B: V \times V \rightarrow V^{\prime}$ is bilinear and continuous. Let us also recall the following properties of the form $b$, see Temam [27], Lemma II.1.3,

$$
b(u, w, v)=-b(u, v, w), \quad u, w, v \in V .
$$

In particular,

$$
b(u, v, v)=0 \quad u, v \in V .
$$

Hence

$$
\langle B(u, w) \mid v\rangle=-\langle B(u, v) \mid w\rangle, \quad u, w, v \in V
$$

and

$$
\langle B(u, v) \mid v\rangle=0, \quad u, v \in V .
$$

Let us, for any $m>0$ define the following standard scale of Hilbert spaces

$$
V_{m}:=\text { the closure of } \mathscr{V} \text { in } H^{m}\left(\mathscr{O}, \mathbb{R}^{d}\right) .
$$

If $m>\frac{d}{2}+1$ then by the Sobolev embedding Theorem, see [1],

$$
H^{m-1}\left(\mathscr{O}, \mathbb{R}^{d}\right) \hookrightarrow \mathscr{C}_{b}\left(\mathscr{O}, \mathbb{R}^{d}\right) \hookrightarrow L^{\infty}\left(\mathscr{O}, \mathbb{R}^{d}\right),
$$

where $\mathscr{C}_{b}\left(\mathscr{O}, \mathbb{R}^{d}\right)$ denotes the space of $\mathbb{R}^{d}$-valued continuous and bounded functions defined on $\mathscr{O}$. If $u, w \in V$ and $v \in V_{m}$ with $m>\frac{d}{2}+1$ then

$$
\begin{aligned}
|b(u, w, v)| & =|b(u, v, w)|=\left|\sum_{i=1}^{n} \int_{\mathscr{O}} u_{i} w \frac{\partial v}{\partial x_{i}} d x\right| \\
& \leq\|u\|_{L^{2}}\|w\|_{L^{2}}\|\nabla v\|_{L^{\infty}} \leq c\|u\|_{L^{2}}\|w\|_{L^{2}}\|v\|_{V_{m}}
\end{aligned}
$$

for some constant $c>0$. Thus, $b$ can be uniquely extented to the three-linear form (denoted by the same letter)

$$
b: H \times H \times V_{m} \rightarrow \mathbb{R}
$$

and $|b(u, w, v)| \leq c\|u\|_{L^{2}}\|w\|_{L^{2}}\|v\| V_{m}$ for $u, w \in H$ and $v \in V_{m}$. At the same time the operator $B$ can be uniquely extended to a bounded bilinear operator

$$
B: H \times H \rightarrow V_{m}^{\prime}
$$

In particular, it satisfies the following estimate

$$
|B(u, w)|_{V_{m}^{\prime}} \leq c|u|_{H}|w|_{H}, \quad u, w \in H .
$$


See Vishik and Fursikov [28]. We will also use the following notation, $B(u):=$ $B(u, u)$. Let us also recall the well known result that the map $B: V \rightarrow V^{\prime}$ is locally Lipschitz continuous, i.e. for every $r>0$ there exists a constant $L_{r}$ such that

$$
|B(u)-B(\tilde{u})|_{V^{\prime}} \leq L_{r}\|u-\tilde{u}\|_{V}, \quad u, \tilde{u} \in V, \quad\|u\|_{V},\|\tilde{u}\|_{V} \leq r .
$$

\subsection{The Space $U$ and Some Operators}

We recall operators and their properties used in [9]. Here we also recall the definition of a Hilbert space $U$ compactly embedded in appropriate space $V_{m}$. This is possible thanks to the result due to Holly and Wiciak, [16] which we recall with the proof in Appendix C, see Lemma 10. This space will be of crucial importance in further investigations.

Consider the natural embedding $j: V \hookrightarrow H$ and its adjoint $j^{*}: H \rightarrow V$. Since the range of $j$ is dense in $H$, the map $j^{*}$ is one-to-one. Let us put

$$
\begin{aligned}
D(A) & :=j^{*}(H) \subset V, \\
A u & :=\left(j^{*}\right)^{-1} u, \quad u \in D(A)
\end{aligned}
$$

and

$$
\mathscr{A} u:=((u \mid \cdot)), \quad u \in V,
$$

where $((\cdot \mid \cdot))$ is defined by Eq. 7. Let us notice that if $u \in V$, then $\mathscr{A} u \in V^{\prime}$ and

$$
|\mathscr{A} u|_{V^{\prime}} \leq\|u\| \text {. }
$$

Indeed, this follows immediately from Eq. 8 and the following inequalities

$$
|((u \mid v))| \leq\|u\| \cdot\|v\| \leq\|u\|\left(\|v\|^{2}+|v|_{H}^{2}\right)^{\frac{1}{2}}=\|u\| \cdot\|v\|_{V}, \quad v \in V .
$$

Lemma 1 (Lemma 2.2 in [9])

(a) For any $u \in D(A)$ and $v \in V$ :

$$
((A-I) u \mid v)_{H}=((u \mid v))=\langle\mathscr{A} u \mid v\rangle,
$$

where I stands for the identity operator on $H$ and $\langle\mid\rangle$ is the standard duality pairing. In particular,

$$
|\mathscr{A} u|_{V^{\prime}} \leq|(A-I) u|_{H}
$$

(b) $D(A)$ is dense in $H$.

Proof To prove assertion (a), let $u \in D(A)$ and $v \in V$. Then

$$
\begin{aligned}
(A u \mid v)_{H} & =\left(\left(j^{*}\right)^{-1} u \mid v\right)_{H}=\left(\left(j^{*}\right)^{-1} u \mid j v\right)_{H}=\left(j^{*}\left(j^{*}\right)^{-1} u \mid v\right)_{V}=(u \mid v)_{V} \\
& =(u \mid v)_{H}+((u \mid v))=(I u \mid v)_{H}+\langle\mathscr{A} u \mid v\rangle .
\end{aligned}
$$

Let us move to the proof of part (b). Since $V$ is dense in $H$, it is sufficient to prove that $D(A)$ is dense in $V$. Let $w \in V$ be an arbitrary element orthogonal to $D(A)$ with respect to the scalar product in $V$. Then

$$
(u \mid w)_{V}=0 \quad \text { for } \quad u \in D(A) .
$$


On the other hand, by (a) and Eq. 6, $(u \mid w)_{V}=(A u \mid w)_{H}$ for $u \in D(A)$. Hence $(A u \mid w)_{H}=0$ for $u \in D(A)$. Since $A: D(A) \rightarrow H$ is onto, we infer that $w=0$, which completes the proof.

Let us assume that $m>1$. It is clear that $V_{m}$ is dense in $V$ and the embedding $j_{m}: V_{m} \hookrightarrow V$ is continuous. Then by Lemma 10 in Appendix $\mathrm{C}$, there exists a Hilbert space $U$ such that $U \subset V_{m}, U$ is dense in $V_{m}$ and

$$
\text { the natural embedding } \iota_{m}: U \hookrightarrow V_{m} \text { is compact . }
$$

Then we have

$$
U \stackrel{l_{m}}{\hookrightarrow} V_{m} \stackrel{j_{m}}{\longrightarrow} V \stackrel{j}{\hookrightarrow} H \cong H^{\prime} \stackrel{j^{\prime}}{\hookrightarrow} V^{\prime} \stackrel{j_{m}^{\prime}}{\longrightarrow} V_{m}^{\prime} \stackrel{i_{m}^{\prime}}{\longrightarrow} U^{\prime}
$$

Since the embedding $\iota_{m}$ is compact, $\iota_{m}^{\prime}$ is compact as well. Consider the composition

$$
\iota:=j \circ j_{m} \circ \iota_{m}: U \hookrightarrow H
$$

and its adjoint

$$
\iota^{*}:=\left(j \circ j_{m} \circ \iota_{m}\right)^{*}=\iota_{m}^{*} \circ j_{m}^{*} \circ j^{*}: H \rightarrow U .
$$

Note that $\iota$ is compact and since the range of $\iota$ is dense in $H, \iota^{*}: H \rightarrow U$ is one-toone. Let us put

$$
\begin{aligned}
D(L) & :=\iota^{*}(H) \subset U, \\
L u & :=\left(\iota^{*}\right)^{-1} u, \quad u \in D(L) .
\end{aligned}
$$

It is clear that $L: D(L) \rightarrow H$ is onto. Let us also notice that

$$
(L u \mid w)_{H}=(u \mid w)_{U}, \quad u \in D(L), \quad w \in U .
$$

By equality (19) and the densiness of $U$ in $H$, we infer similarly as in the proof of assertion (b) in Lemma 1 that $D(L)$ is dense in $H$. Moreover, for $u \in D(L)$,

$$
L u=\left(\iota^{*}\right)^{-1} u=\left(\iota_{m}^{*} \circ j_{m}^{*} \circ j^{*}\right)^{-1} u=A \circ\left(j_{m}^{*}\right)^{-1} \circ\left(\iota_{m}^{*}\right)^{-1} u,
$$

where $A$ is defined by Eq. 14 .

Since $L$ is self-adjoint and $L^{-1}$ is compact, there exists an orthonormal basis $\left\{e_{i}\right\}_{i \in \mathbb{N}}$ of $H$ composed of the eigenvectors of operator $L$. Let us fix $n \in \mathbb{N}$ and let $P_{n}$ be the operator from $U^{\prime}$ to $\operatorname{span}\left\{e_{1}, \ldots, e_{n}\right\}$ defined by

$$
P_{n} u^{*}:=\sum_{i=1}^{n}\left\langle u^{*} \mid e_{i}\right\rangle e_{i}, \quad u^{*} \in U^{\prime},
$$

where $\langle\cdot \mid \cdot\rangle$ denotes the duality pairing between the space $U$ and its dual $U^{\prime}$. Note that the restriction of $P_{n}$ to $H$, denoted still by $P_{n}$, is given by

$$
P_{n} u=\sum_{i=1}^{n}\left(u \mid e_{i}\right)_{H} e_{i}, \quad u \in H,
$$


and thus it is the $(\cdot \mid \cdot)_{H}$-orthogonal projection onto $\operatorname{span}\left\{e_{1}, \ldots, e_{n}\right\}$. Restrictions of $P_{n}$ to other spaces considered in Eq. 17 will also be denoted by $P_{n}$. Moreover, it is easy to see that

$$
\left(P_{n} u^{*} \mid v\right)_{H}=\left\langle u^{*} \mid P_{n} v\right\rangle, \quad u^{*} \in U^{\prime}, \quad v \in U
$$

It is easy to prove that the system $\left\{\frac{e_{i}}{\left\|e_{i}\right\| U}\right\}_{n \in \mathbb{N}}$ is the $(\cdot \mid \cdot)_{U}$-orthonormal basis in the space $U$ and that the restriction of $P_{n}$ to $U$ is the $(\cdot \mid \cdot)_{U}$-projection onto the subspace $\operatorname{span}\left\{e_{1}, \ldots, e_{n}\right\}$. In particular, for every $u \in U$

(i) $\lim _{n \rightarrow \infty}\left\|P_{n} u-u\right\|_{U}=0$,

(ii) $\lim _{n \rightarrow \infty}\left\|P_{n} u-u\right\|_{V_{m}}=0$, where $m>0$,

(iii) $\lim _{n \rightarrow \infty}\left\|P_{n} u-u\right\|_{V}=0$.

See Lemma 2.4 in [9] for details.

We will use the basis $\left\{e_{i}\right\}_{i \in \mathbb{N}}$ and the operators $P_{n}$ in the Faedo-Galerkin approximation.

\section{Compactness Results}

\subsection{The Space of Càdlàg Functions}

Let $(\mathbb{S}, \rho)$ be a separable and complete metric space. Let $\mathbb{D}([0, T] ; \mathbb{S})$ the space of all $\mathbb{S}$-valued càdlàg functions defined on $[0, T]$, i.e. the functions which are right continuous and have left limits at every $t \in[0, T]$. The space $\mathbb{D}([0, T] ; \mathbb{S})$ is endowed with the Skorokhod topology.

Remark 1 A sequence $\left(u_{n}\right) \subset \mathbb{D}([0, T] ; \mathbb{S})$ converges to $u \in \mathbb{D}([0, T] ; \mathbb{S})$ iff there exists a sequence $\left(\lambda_{n}\right)$ of homeomorphisms of $[0, T]$ such that $\lambda_{n}$ tends to the identity uniformly on $[0, T]$ and $u_{n} \circ \lambda_{n}$ tends to $u$ uniformly on $[0, T]$.

This topology is metrizable by the following metric $\delta_{T}$

$$
\delta_{T}(u, v):=\inf _{\lambda \in \Lambda_{T}}\left[\sup _{t \in[0, T]} \rho(u(t), v \circ \lambda(t))+\sup _{t \in[0, T]}|t-\lambda(t)|+\sup _{s \neq t}\left|\log \frac{\lambda(t)-\lambda(s)}{t-s}\right|\right],
$$

where $\Lambda_{T}$ is the set of increasing homeomorphisms of $[0, T]$. Moreover, $\left(\mathbb{D}([0, T] ; \mathbb{S}), \delta_{T}\right)$ is a complete metric space, see [19].

Let us recall the notion of a modulus of the function. It plays analogous role in the space $\mathbb{D}([0, T] ; \mathbb{S})$ as the modulus of continuity in the space of continuous functions $\mathbb{C}([0, T] ; \mathbb{S})$.

Definition 1 (see [22]) Let $u \in \mathbb{D}([0, T] ; \mathbb{S})$ and let $\delta>0$ be given. A modulus of $u$ is defined by

$$
w_{[0, T], \mathbb{S}}(u, \delta):=\inf _{\Pi_{\delta}} \max _{t_{i} \in \bar{\omega}} \sup _{t_{i} \leq s<t<t_{i+1} \leq T} \rho(u(t), u(s)),
$$


where $\Pi_{\delta}$ is the set of all increasing sequences $\bar{\omega}=\left\{0=t_{0}<t_{1}<\ldots<t_{n}=T\right\}$ with the following property

$$
t_{i+1}-t_{i} \geq \delta, \quad i=0,1, \ldots, n-1 .
$$

If no confusion seems likely, we will denote the modulus by $w_{[0, T]}(u, \delta)$.

We have the following criterion for relative compactness of a subset of the space $\mathbb{D}([0, T] ; \mathbb{S})$, see $[19,22]$, Ch.II, and [4], Ch.3, analogous to the Arzelà-Ascoli Theorem for the space of continuous functions.

Theorem 1 A set $A \subset \mathbb{D}([0, T] ; \mathbb{S})$ has compact closure iff it satisfies the following two conditions:

(a) there exists a dense subset $J \subset[0, T]$ such that for every $t \in J$ the $\operatorname{set}\{u(t), u \in A\}$ has compact closure in $\mathbb{S}$.

(b) $\lim _{\delta \rightarrow 0} \sup _{u \in A} w_{[0, T]}(u, \delta)=0$.

\subsection{Deterministic Compactness Criterion}

Let us recall that $V$ and $H$ are Hilbert spaces defined by Eqs. 4-8. Since $\mathscr{O}$ is an arbitrary domain of $\mathbb{R}^{d},(d=2,3)$, the embedding $V \hookrightarrow H$ is dense and continuous. We have defined a Hilbert space $U \subset V$ such that the embedding $U \hookrightarrow V$ is dense and compact, see Eq. 16. In particular, we have

$$
U \hookrightarrow V \hookrightarrow H \cong H^{\prime} \hookrightarrow U^{\prime}
$$

the embedding $H \hookrightarrow U^{\prime}$ being compact as well. Let $\left(\mathscr{O}_{R}\right)_{R \in \mathbb{N}}$ be a sequence of open and bounded subsets of $\mathscr{O}$ with regular boundaries $\partial \mathscr{O}_{R}$ such that $\mathscr{O}_{R} \subset \mathscr{O}_{R+1}$ and $\bigcup_{R=1}^{\infty} \mathscr{O}_{R}=\mathscr{O}$. We will consider the following spaces of restrictions of functions defined on $\mathscr{O}$ to subsets $\mathscr{O}_{R}$, i.e.

$$
H_{\mathscr{O}_{R}}:=\left\{u_{\mid \mathscr{O}_{R}} ; \quad u \in H\right\} \quad V_{\mathscr{O}_{R}}:=\left\{v_{\mid \mathscr{O}_{R}} ; \quad v \in V\right\}
$$

with appropriate scalar products and norms, i.e.

$$
\begin{aligned}
(u \mid v)_{H_{\mathscr{O}_{R}}}: & =\int_{\mathscr{O}_{R}} u v d x, \quad u, v \in H_{\mathscr{O}_{R}}, \\
(u \mid v)_{V_{\mathscr{O}_{R}}}: & =\int_{\mathscr{O}_{R}} u v d x+\int_{\mathscr{O}_{R}} \nabla u \nabla v d x, \quad u, v \in V_{\mathscr{O}_{R}}
\end{aligned}
$$

and $|u|_{H_{\mathscr{O}_{R}}}^{2}:=(u \mid u)_{H_{\mathscr{O}_{R}}}$ for $u \in H_{\mathscr{O}_{R}}$ and $\|u\|_{V_{\mathscr{O}_{R}}}^{2}:=(u \mid u)_{V_{\mathscr{O}_{R}}}$ for $u \in V_{\mathscr{O}_{R}}$. The symbols $H_{\mathscr{O}_{R}}^{\prime}$ and $V_{\mathscr{O}_{R}}^{\prime}$ will stand for the corresponding dual spaces.

Since the sets $\mathscr{O}_{R}$ are bounded,

$$
\text { the embeddings } V_{\mathscr{O}_{R}} \hookrightarrow H_{\mathscr{O}_{R}} \text { are compact. }
$$


Let $q \in(1, \infty)$. Let us consider the following three functional spaces, analogous to those considered in [25] and [9], see also [22, 23]:

$$
\begin{aligned}
\mathbb{D}\left([0, T], U^{\prime}\right):= & \text { the space of càdlàg functions } u:[0, T] \rightarrow U^{\prime} \text { with the } \\
& \text { topology } \mathscr{T}_{1} \text { induced by the Skorokhod metric } \delta_{T}, \\
L_{w}^{q}(0, T ; V):= & \text { the space } L^{q}(0, T ; V) \text { with the weak topology } \mathscr{T}_{2}, \\
L^{q}\left(0, T ; H_{\text {loc }}\right):= & \text { the space of measurable functions } u:[0, T] \rightarrow H \\
& \text { such that for all } R \in \mathbb{N}
\end{aligned}
$$$$
p_{T, R}(u):=\|u\|_{L^{q}\left(0, T ; H_{\mathscr{O}_{R}}\right)}:=\left(\int_{0}^{T} \int_{\mathscr{O}_{R}}|u(t, x)|^{q} d x d t\right)^{\frac{1}{q}}<\infty,
$$

with the topology $\mathscr{T}_{3}$ generated by the seminorms

$$
\left(p_{T, R}\right)_{R \in \mathbb{N}} \text {. }
$$

Let $H_{w}$ denote the Hilbert space $H$ endowed with the weak topology. Let us consider the fourth space, see [25],

$\mathbb{D}\left([0, T] ; H_{w}\right):=$ the space of weakly càdlàg functions $u:[0, T] \rightarrow H$ with the weakest topology $\mathscr{T}_{4}$ such that for all $h \in h$ the mappings

$$
\mathbb{D}\left([0, T] ; H_{w}\right) \ni u \mapsto(u(\cdot) \mid h)_{H} \in \mathbb{D}([0, T] ; \mathbb{R}) \text { are continuous. }
$$

In particular, $u_{n} \rightarrow u$ in $\mathbb{D}\left([0, T] ; H_{w}\right)$ iff for all $h \in H$ :

$$
\left(u_{n}(\cdot) \mid h\right)_{H} \rightarrow(u(\cdot) \mid h)_{H} \quad \text { in the space } \quad \mathbb{D}([0, T] ; \mathbb{R}) .
$$

Let us consider the ball

$$
\mathbb{B}:=\left\{x \in H:|x|_{H} \leq r\right\} .
$$

Let $\mathbb{B}_{w}$ denote the ball $\mathbb{B}$ endowed with the weak topology. It is well-known that the $\mathbb{B}_{w}$ is metrizable, see [5]. Let $q_{r}$ denote the metric compatible with the weak topology on $\mathbb{B}$. Let us consider the following space

$$
\begin{gathered}
\mathbb{D}\left([0, T] ; \mathbb{B}_{w}\right)=\text { the space of weakly càdlàg functions } u:[0, T] \rightarrow H \\
\text { and such that } \sup _{t \in[0, T]}|u(t)|_{H} \leq r .
\end{gathered}
$$

Then $\mathbb{D}\left([0, T] ; \mathbb{B}_{w}\right)$ is metrizable with

$$
\delta_{T, r}(u, v)=\inf _{\lambda \in \Lambda_{T}}\left\{\sup _{t \in[0, T]} q_{r}(u(t), v \circ \lambda(t))+\sup _{t \in[0, T]}|t-\lambda(t)|+\sup _{s \neq t}\left|\log \frac{\lambda(t)-\lambda(s)}{t-s}\right|\right\} .
$$

Since by the Banach-Alaoglu Theorem $\mathbb{B}_{w}$ is compact, $\left(\mathbb{D}\left([0, T] ; \mathbb{B}_{w}\right), \delta_{T, r}\right)$ is a complete metric space.

The following lemma says that any sequence $\left(u_{n}\right) \subset L^{\infty}(0, T ; H)$ convergent in $\mathbb{D}\left([0, T] ; U^{\prime}\right)$ is also convergent in the space $\mathbb{D}\left([0, T] ; \mathbb{B}_{w}\right)$. 
Lemma 2 (see Lemma 4.3 in [25]) Let $u_{n}:[0, T] \rightarrow H, n \in \mathbb{N}$, be functions such that

(i) $\sup _{n \in \mathbb{N}} \sup _{s \in[0, T]}\left|u_{n}(s)\right|_{H} \leq r$,

(ii) $u_{n} \rightarrow u$ in $\mathbb{D}\left([0, T] ; U^{\prime}\right)$.

Then $u, u_{n} \in \mathbb{D}\left([0, T] ; \mathbb{B}_{w}\right)$ and $u_{n} \rightarrow$ u in $\mathbb{D}\left([0, T] ; \mathbb{B}_{w}\right)$ as $n \rightarrow \infty$.

We recall the proof in Appendix E.

The following Theorem is a generalization of the results of [9] and [25]. In the paper [25] the analogous result is proved in the case when the embedding $V \subset H$ is dense and compact. In [9] the embedding $V \subset H$ is only dense and continuous. However, instead of the spaces of càdlàg functions, appropriate spaces of continuous functions are used. The following result generalizes both [9] and [25] in the sense that the embedding $V \subset H$ is dense and continuous and appropriate spaces of càdlàg functions are considered, i.e. $\mathbb{D}\left([0, T] ; U^{\prime}\right)$ and $\mathbb{D}\left([0, T], H_{w}\right)$.

Theorem 2 Let $q \in(1, \infty)$ and let

$$
\mathscr{Z}_{q}:=L_{w}^{q}(0, T ; V) \cap L^{q}\left(0, T ; H_{\mathrm{loc}}\right) \cap \mathbb{D}\left([0, T] ; U^{\prime}\right) \cap \mathbb{D}\left([0, T], H_{w}\right)
$$

and let $\mathscr{T}$ be the supremum of the corresponding topologies. Then a set $\mathscr{K} \subset \mathscr{Z}_{q}$ is $\mathscr{T}$-relatively compact if the following three conditions hold

(a) for all $u \in \mathscr{K}$ and all $t \in[0, T], u(t) \in H$ and $\sup _{u \in \mathscr{K}} \sup _{s \in[0, T]}|u(s)|_{H}<\infty$,

(b) $\sup _{u \in \mathscr{K}} \int_{0}^{T}\|u(s)\|_{V}^{q} d s<\infty$, i.e. $\mathscr{K}$ is bounded in $L^{q}(0, T ; V)$,

(c) $\lim _{\delta \rightarrow 0} \sup _{u \in \mathscr{K}} w_{[0, T], U^{\prime}}(u ; \delta)=0$.

Proof We can assume that $\mathscr{K}$ is a closed subset of $\mathscr{Z}_{q}$. Because of the assumption (b), the weak topology in $L_{w}^{q}(0, T ; V)$ induced on $\mathscr{Z}_{q}$ is metrizable. Since the topology in $L^{q}\left(0, T ; H_{\text {loc }}\right)$ is defined by the countable family of seminorms (24), this space is also metrizable. By assumption (a), it is sufficient to consider the metric subspace $\mathbb{D}\left([0, T] ; \mathbb{B}_{w}\right) \subset \mathbb{D}\left([0, T], H_{w}\right)$ defined by Eqs. 26 and 27 with $r:=\sup _{u \in \mathscr{K}}$ $\sup _{s \in[0, T]}|u(s)|_{H}$. Thus compactness of a subset of $\mathscr{Z}_{q}$ is equivalent to its sequential compactness. Let $\left(u_{n}\right)$ be a sequence in $\mathscr{K}$. By the Banach-Alaoglu Theorem condition (b) yields that the set $\mathscr{K}$ is compact in $L_{w}^{q}(0, T ; V)$.

Using the compactness criterion in the space of càdlàg functions contained in Theorem 1, we will prove that $\left(u_{n}\right)$ is compact in $\mathbb{D}\left([0, T] ; U^{\prime}\right)$. Indeed, by (a) for every $t \in[0, T]$ the set $\left\{u_{n}(t), n \in \mathbb{N}\right\}$ is bounded in $H$. Since the embedding $H \subset U^{\prime}$ is compact, the set $\left\{u_{n}(t), n \in \mathbb{N}\right\}$ is compact in $U^{\prime}$. This together with condition (c) implies compactness of the sequence $\left(u_{n}\right)$ in the space $\mathbb{D}\left([0, T] ; U^{\prime}\right)$.

Therefore there exists a subsequence $\left(u_{n_{k}}\right) \subset\left(u_{n}\right)$ such that

$$
u_{n_{k}} \rightarrow u \quad \text { in } \quad L_{w}^{q}(0, T ; V) \cap \mathbb{D}\left([0, T] ; U^{\prime}\right) \quad \text { as } \quad k \rightarrow \infty .
$$

Since $u_{n_{k}} \rightarrow u$ in $\mathbb{D}\left([0, T] ; U^{\prime}\right), u_{n_{k}}(t) \rightarrow u(t)$ in $U^{\prime}$ for all continuity points of function $u$, (see [4]). By condition (a) and the Lebesgue dominated convergence theorem, we infer that for all $p \in[1, \infty)$

$$
u_{n_{k}} \rightarrow u \quad \text { in } \quad L^{p}\left(0, T ; U^{\prime}\right) \quad \text { as } \quad k \rightarrow \infty .
$$

We claim that

$$
u_{n_{k}} \rightarrow u \quad \text { in } \quad L^{q}\left(0, T ; H_{\mathrm{loc}}\right) \quad \text { as } \quad k \rightarrow \infty .
$$


In order to prove it let us fix $R>0$. Since, by Eq. 23 the embedding $V_{\mathscr{O}_{R}} \hookrightarrow H_{\mathscr{O}_{R}}$ is compact and the embeddings $H_{\mathscr{O}_{R}} \hookrightarrow H^{\prime} \hookrightarrow U^{\prime}$ are continuous, by the Lions Lemma, [20], for every $\varepsilon>0$ there exists a costant $C=C_{\varepsilon, R}>0$ such that

$$
|u|_{H_{\mathscr{O}_{R}}}^{q} \leq \varepsilon\|u\|_{V_{\mathscr{O}_{R}}}^{q}+C_{\varepsilon}|u|_{U^{\prime}}^{q}, \quad u \in V .
$$

Thus for almost all $s \in[0, T]$

$$
\left|u_{n_{k}}(s)-u(s)\right|_{H_{\mathscr{O}_{R}}}^{q} \leq \varepsilon\left\|u_{n_{k}}(s)-u(s)\right\|_{V_{\mathscr{O}_{R}}}^{q}+C_{\varepsilon}\left|u_{n_{k}}(s)-u(s)\right|_{U^{\prime}}^{q}, \quad k \in \mathbb{N},
$$

and so for all $k \in \mathbb{N}$

$$
\left\|u_{n_{k}}-u\right\|_{L^{q}\left(0, T ; H_{\mathscr{O}_{R}}\right)}^{q} \leq \varepsilon\left\|u_{n_{k}}-u\right\|_{L^{q\left(0, T ; V_{\mathscr{O}_{R}}\right)}}^{q}+C_{\varepsilon}\left\|u_{n_{k}}-u\right\|_{L^{q}\left(0, T ; U^{\prime}\right)}^{q} .
$$

Passing to the upper limit as $k \rightarrow \infty$ in the above inequality and using the estimate

$$
\left\|u_{n_{k}}-u\right\|_{L^{q}\left(0, T ; V_{\mathscr{O}_{R}}\right)}^{q} \leq q\left(\left\|u_{n_{k}}\right\|_{L^{q}\left(0, T ; V_{\mathscr{O}_{R}}\right)}^{q}+\|u\|_{L^{q}\left(0, T ; V_{\mathscr{O}_{R}}\right)}^{q}\right) \leq 2 q c_{q},
$$

where $c_{q}=\sup _{u \in \mathscr{K}}\|u\|_{L^{q}(0, T ; V)}^{q}$, we infer that

$$
\limsup _{k \rightarrow \infty}\left\|u_{n_{k}}-u\right\|_{L^{q}\left(0, T ; H_{\mathscr{O}_{R}}\right)}^{q} \leq 2 q c_{q} \varepsilon
$$

By the arbitrariness of $\varepsilon$,

$$
\lim _{k \rightarrow \infty}\left\|u_{n_{k}}-u\right\|_{L^{q}\left(0, T ; H_{\mathscr{O}_{R}}\right)}^{q}=0 .
$$

The proof of Theorem is thus complete.

\subsection{Tightness Criterion}

Let us recall that $U, V, H$ are separable Hilbert spaces such that

$$
U \hookrightarrow V \hookrightarrow H,
$$

where the embedding $U \hookrightarrow V$ is compact and $V \hookrightarrow H$ is continuous. Using the compactness criterion formulated in Theorem 2 we obtain the corresponding tightness criterion in the space $\mathscr{Z}_{q}$. Let us first recall that the space $\mathscr{Z}_{q}$ is defined by

$$
\mathscr{Z}_{q}:=L_{w}^{q}(0, T ; V) \cap L^{q}\left(0, T ; H_{\mathrm{loc}}\right) \cap \mathbb{D}\left([0, T] ; U^{\prime}\right) \cap \mathbb{D}\left([0, T], H_{w}\right)
$$

and it is equipped with the topology $\mathscr{T}$, see Eq. 28.

Corollary 1 (Tightness Criterion) Let $\left(X_{n}\right)_{n \in \mathbb{N}}$ be a sequence of càdlàg $\mathbb{F}$-adapted $U^{\prime}$ valued processes such that

(a) there exists a positive constant $C_{1}$ such that

$$
\sup _{n \in \mathbb{N}} \mathbb{E}\left[\sup _{s \in[0, T]}\left|X_{n}(s)\right|_{H}\right] \leq C_{1},
$$

(b) there exists a positive constant $C_{2}$ such that

$$
\sup _{n \in \mathbb{N}} \mathbb{E}\left[\int_{0}^{T}\left\|X_{n}(s)\right\| V q d s\right] \leq C_{2},
$$


(c) $\left(X_{n}\right)_{n \in \mathbb{N}}$ satisfies the Aldous condition $[\mathbf{A}]$ in $U^{\prime}$.

Let $\tilde{\mathbb{P}}_{n}$ be the law of $X_{n}$ on $\mathscr{Z}_{q}$. Then for every $\varepsilon>0$ there exists a compact subset $K_{\varepsilon}$ of $\mathscr{Z}_{q}$ such that

$$
\tilde{\mathbb{P}}_{n}\left(K_{\varepsilon}\right) \geq 1-\varepsilon
$$

We recall the Aldous condition [A] in Appendix A, see Definition 5. The proof of Corrollary 1 is postponed to Appendix A, as well.

\section{Stochastic Navier-Stokes Equations Driven by Lévy Noise}

\subsection{Time Homogeneous Poisson Random Measure}

We follow the approach due to Brzeźniak and Hausenblas [6, 7], see also [17] and [26]. Let us denote $\mathbb{N}:=\{0,1,2, \ldots\}, \overline{\mathbb{N}}:=\mathbb{N} \cup\{\infty\}, \mathbb{R}_{+}:=[0, \infty)$. Let $(S, \mathscr{S})$ be a measurable space and let $M_{\overline{\mathbb{N}}}(S)$ be the set of all $\overline{\mathbb{N}}$ valued measures on $(S, \mathscr{S})$. On the set $M_{\overline{\mathbb{N}}}(S)$ we consider the $\sigma$-field $\mathscr{M}_{\overline{\mathbb{N}}}(S)$ defined as the smallest $\sigma$-field such that for all $B \in \mathscr{S}$ : the map

$$
i_{B}: M_{\overline{\mathbb{N}}}(S) \ni \mu \mapsto \mu(B) \in \overline{\mathbb{N}}
$$

is measurable.

Let $(\Omega, \mathscr{F}, \mathbb{P})$ be a complete probability space with filtration $\mathbb{F}:=\left(\mathscr{F}_{t}\right)_{t \geq 0}$ satisfying the usual hypotheses, see [21].

Definition 2 (see Appendix $C$ in [7]) Let $(Y, \mathscr{Y})$ be a measurable space. A time homogeneous Poisson random measure $\eta$ on $(Y, \mathscr{Y})$ over $(\Omega, \mathscr{F}, \mathbb{F}, \mathbb{P})$ is a measurable function

$$
\eta:(\Omega, \mathscr{F}) \rightarrow\left(M_{\overline{\mathbb{N}}}\left(\mathbb{R}_{+} \times Y\right), \mathscr{M}_{\overline{\mathbb{N}}}\left(\mathbb{R}_{+} \times Y\right)\right)
$$

such that

(1) for all $B \in \mathscr{B}\left(\mathbb{R}_{+}\right) \otimes \mathscr{Y}, \eta(B):=i_{B} \circ \eta: \Omega \rightarrow \overline{\mathbb{N}}$ is a Poisson random measure with parameter $\mathbb{E}[\eta(B)]$;

(2) $\quad \eta$ is independently scattered, i.e. if the sets $B_{j} \in \mathscr{B}\left(\mathbb{R}_{+}\right) \otimes \mathscr{Y}, j=1, \ldots, n$, are disjoint then the random variables $\eta\left(B_{j}\right), j=1, \ldots, n$, are independent;

(3) for all $U \in \mathscr{Y}$ the $\overline{\mathbb{N}}$-valued process $(N(t, U))_{t \geq 0}$ defined by

$$
N(t, U):=\eta((0, t] \times U), \quad t \geq 0
$$

is $\mathbb{F}$-adapted and its increments are independent of the past, i.e. if $t>s \geq 0$, then $N(t, U)-N(s, U)=\eta((s, t] \times U)$ is independent of $\mathscr{F}_{s}$.

If $\eta$ is a time homogeneous Poisson random measure then the formula

$$
v(A):=\mathbb{E}[\eta((0,1] \times A)], \quad A \in \mathscr{Y}
$$


defines a measure on $(Y, \mathscr{Y})$ called an intensity measure of $\eta$. Moreover, for all $T<\infty$ and all $A \in \mathscr{Y}$ such that $\mathbb{E}[\eta((0, T] \times A)]<\infty$, the $\mathbb{R}$-valued process $\{\tilde{N}(t, A)\}_{t \in(0, T]}$ defined by

$$
\tilde{N}(t, A):=\eta((0, t] \times A)-t v(A), \quad t \in(0, T],
$$

is an integrable martingale on $(\Omega, \mathscr{F}, \mathbb{F}, \mathbb{P})$. The random measure $l \otimes v$ on $\mathscr{B}\left(\mathbb{R}_{+}\right)$ $\otimes \mathscr{Y}$, where $l$ stands for the Lebesgue measure, is called an compensator of $\eta$ and the difference between a time homogeneous Poisson random measure $\eta$ and its compensator, i.e.

$$
\tilde{\eta}:=\eta-l \otimes v
$$

is called a compensated time homogeneous Poisson random measure.

Let us also recall basic properties of the stochastic integral with respect to $\tilde{\eta}$, see $[7,17]$ and [26] for details. Let $\mathbb{H}$ be a separable Hilbert space and let $\mathscr{P}$ be a predictable $\sigma$-field on $[0, T] \times \Omega$. Let $\mathfrak{L}_{v, T}^{2}(\mathscr{P} \otimes \mathscr{Y}, l \otimes \mathbb{P} \otimes v ; \mathbb{H})$ be a space of all $\mathbb{H}$-valued, $\mathscr{P} \otimes \mathscr{Y}$-measurable processes such that

$$
\mathbb{E}\left[\int_{0}^{T} \int_{Y}\|\xi(s, \cdot, y)\|_{\mathbb{H}}^{2} d s d v(y)\right]<\infty
$$

If $\xi \in \mathfrak{L}_{v, T}^{2}(\mathscr{P} \otimes \mathscr{Y}, l \otimes \mathbb{P} \otimes v ; \mathbb{H})$ then the integral process $\int_{0}^{t} \int_{Y} \xi(s, \cdot, y) \tilde{\eta}(d s, d y)$, $t \in[0, T]$, is a càdlàg $L^{2}$-integrable martingale. Moreover, the following isometry formula holds

$$
\mathbb{E}\left[\left\|\int_{0}^{t} \int_{Y} \xi(s, \cdot, y) \tilde{\eta}(d s, d y)\right\|_{\mathbb{H}}^{2}\right]=\mathbb{E}\left[\int_{0}^{t} \int_{Y}\|\xi(s, \cdot, y)\|_{\mathbb{H}}^{2} d s d v(y)\right], t \in[0, T] .
$$

4.2 Statement of the Problem

Problem (1-3) can be written as the following stochastic evolution equation

$$
\begin{gathered}
d u(t)+[\mathscr{A} u(t)+B(u(t))] d t=f(t) d t+\int_{Y} F(t, u(t) ; y) \tilde{\eta}(d t, d y) \\
+G(t, u(t)) d W(t), \quad t \in[0, T] \\
u(0)=u_{0} .
\end{gathered}
$$

Assumption We assume that

(A.1) $u_{0} \in H, f \in L^{2}\left([0, T] ; V^{\prime}\right)$,

(F.1) $\tilde{\eta}$ is a compensated time homogeneous Poisson random measure on a measurable space $(Y, \mathscr{Y})$ over $(\Omega, \mathscr{F}, \mathbb{F}, \mathbb{P})$ with a $\sigma$-finite intensity measure $v$,

(F.2) $F:[0, T] \times H \times Y \rightarrow H$ is a measurable function such that $\int_{Y} 11_{\{0\}}(F(t, x ; y)) v(d y)=0$ for all $x \in H$ and $t \in[0, T]$. Moreover, there exists a constant $L$ such that

$$
\int_{Y}\left|F\left(t, u_{1} ; y\right)-F\left(t, u_{2} ; y\right)\right|_{H}^{2} v(d y) \leq L\left|u_{1}-u_{2}\right|_{H}^{2}, \quad u_{1}, u_{2} \in H, t \in[0, T],
$$


and for each $p \in\{2,4,4+\gamma, 8+2 \gamma\}$ there exists a constant $C_{p}$ such that

$$
\int_{Y}|F(t, u ; y)|_{H}^{p} v(d y) \leq C_{p}\left(1+|u|_{H}^{p}\right), \quad u \in H, \quad t \in[0, T],
$$

where $\gamma>0$ is some positive constant.

(F.3) Moreover, for all $v \in \mathscr{V}$ the mapping $\tilde{F}_{v}$ defined by

$$
\left(\tilde{F}_{v}(u)\right)(t, y):=\left(F\left(t, u\left(t^{-}\right) ; y\right) \mid v\right)_{H}, \quad u \in L^{2}(0, T ; H), \quad(t, y) \in[0, T] \times Y
$$

is a continuous from $L^{2}(0, T ; H)$ into $L^{2}([0, T] \times Y, d l \otimes v ; \mathbb{R})$ if in the space $L^{2}(0, T ; H)$ we consider the Fréchet topology inherited from the space $L^{2}\left(0, T ; H_{\text {loc }}\right){ }^{1}$

(G.1) $W(t)$ is a cylindrical Wiener process in a separable Hilbert space $Y_{W}$ defined on the stochastic basis $(\Omega, \mathscr{F}, \mathbb{F}, \mathbb{P})$;

(G.2) $G:[0, T] \times V \rightarrow \mathscr{L}_{\mathrm{HS}}\left(Y_{W}, H\right)$ and there exists a constant $L_{G}>0$ such that

$$
\left\|G\left(t, u_{1}\right)-G\left(t, u_{2}\right)\right\|_{\mathscr{L}_{\mathrm{HS}}\left(Y_{W}, H\right)}^{2} \leq L_{G}\left\|u_{1}-u_{2}\right\|_{V}^{2}, \quad u_{1}, u_{2} \in V, t \in[0, T] .
$$

Moreover there exist $\lambda, \kappa \in \mathbb{R}$ and $a \in\left(2-\frac{2}{3+\gamma}, 2\right]$ such that

$$
2\langle\mathscr{A} u \mid u\rangle-\|G(t, u)\|_{\mathscr{L}_{\mathrm{HS}}\left(Y_{W}, H\right)}^{2} \geq a\|u\| 2-\lambda|u|_{H}^{2}-\kappa, \quad u \in V, t \in[0, T] .
$$

(G.3) Moreover, $G$ extends to a continuous mapping $G:[0, T] \times H \rightarrow$ $\mathscr{L}_{\mathrm{HS}}\left(Y_{W}, V^{\prime}\right)$ such that

$$
\|G(t, u)\|_{\mathscr{L}_{\mathrm{HS}}\left(Y_{W}, V^{\prime}\right)}^{2} \leq C\left(1+|u|_{H}^{2}\right), \quad u \in H .
$$

for some $C>0$. Moreover, for every $v \in \mathscr{V}$ the mapping $\tilde{G}_{v}$ defined by

$$
\left(\tilde{G}_{v}(u)\right)(t):=(G(t, u(t)) \mid v)_{H}, \quad u \in L^{2}(0, T ; H), \quad t \in[0, T]
$$

is a continuous mapping from $L^{2}(0, T ; H)$ into $L^{2}\left([0, T] ; \mathscr{L}_{\mathrm{HS}}\left(Y_{W}, \mathbb{R}\right)\right)$ if in the space $L^{2}(0, T ; H)$ we consider the Fréchet topology inherited from the space $L^{2}\left(0, T ; H_{\text {loc }}\right)$.

Let us recall that the space $L^{2}\left(0, T ; H_{\text {loc }}\right)$ is defined by Eq. 24. For any Hilbert space $E$ the symbol $\mathscr{L}_{\mathrm{HS}}\left(Y_{W} ; E\right)$ denotes the space of Hilbert-Schmidt operators from $Y_{W}$ into $E$.

Definition 3 A martingale solution of Eq. 30 is a system $(\bar{\Omega}, \overline{\mathscr{F}}, \overline{\mathbb{F}}, \overline{\mathbb{P}}, \bar{u}, \bar{\eta}, \bar{W})$, where

- $(\bar{\Omega}, \overline{\mathscr{F}}, \overline{\mathbb{F}}, \overline{\mathbb{P}})$ is a filtered probability space with a filtration $\overline{\mathbb{F}}=\left\{\overline{\mathscr{F}}_{t}\right\}_{t \geq 0}$,

- $\quad \bar{\eta}$ is a time homogeneous Poisson random measure on $(Y, \mathscr{Y})$ over $(\bar{\Omega}, \overline{\mathscr{F}}, \overline{\mathbb{F}}, \overline{\mathbb{P}})$ with the intensity measure $v$,

- $\bar{W}$ is a cylindrical Wiener process on the space $Y_{W}$ over $(\bar{\Omega}, \overline{\mathscr{F}}, \overline{\mathbb{F}}, \overline{\mathbb{P}})$,

\footnotetext{
${ }^{1}$ Here $l$ denotes the Lebesgue measure on the interval $[0, T]$.
} 
- $\quad \bar{u}:[0, T] \times \Omega \rightarrow H$ is a predictable process with $\overline{\mathbb{P}}$-a.e. paths

$$
\bar{u}(\cdot, \omega) \in \mathbb{D}\left([0, T], H_{w}\right) \cap L^{2}(0, T ; V)
$$

such that for all $t \in[0, T]$ and all $v \in V$ the following identity holds $\overline{\mathbb{P}}$-a.s.

$$
\begin{aligned}
(\bar{u}(t) v)_{H} & +\int_{0}^{t}\langle\mathscr{A} \bar{u}(s) \mid v\rangle d s+\int_{0}^{t}\langle B(\bar{u}(s)) \mid v\rangle d s \\
=\left(u_{0} \mid v\right)_{H} & +\int_{0}^{t}\langle f(s) \mid v\rangle d s+\int_{0}^{t} \int_{Y}(F(s, \bar{u}(s) ; y) \mid v)_{H} \tilde{\bar{\eta}}(d s, d y) \\
& +\left\langle\int_{0}^{t} G(s, \bar{u}(s)) d \bar{W}(s) v\right\rangle .
\end{aligned}
$$

We will prove existence of a martingale solution of the Eq. 30. To this end we use the Faedo-Galerkin method. The Galerkin approximations generate a sequence of probability measures on appropriate functional space. We will prove that this sequence is tight. Let us emphasize that to prove the tightness, assumption (F.2) with $p=2$ in inequality (32) is sufficient. The stronger condition on $p$, i.e. inequality (32) for a certain $p>4$, is connected with the construction of the process $\bar{u}$ to deal with the nonlinear term. Assumptions (G.2)-(G.3) allow to consider the Gaussian noise term $G$ dependent both on $u$ and $\nabla u$. This corresponds to inequality (35) with $a<2$. The case when $a=2$ is related to the noise term $G$ dependent on $u$ but not on its gradient. Moreover, assumptions (F.3) and (G.3) are important in the case of unbounded domain $\mathscr{O}$. In the case when $\mathscr{O}$ is bounded, they can be omitted, see [25].

\section{Existence of Solutions}

Theorem 3 There exists a martingale solution of the problem (30) provided assumptions (A.1), (F.1)-(F.3) and (G.1)-(G.3) are satisfied.

\subsection{Faedo-Galerkin Approximation}

Let $\left\{e_{i}\right\}_{i=1}^{\infty}$ be the orthonormal basis in $H$ composed of eigenvectors of the operator $L$ defined by Eq. 18. Let $H_{n}:=\operatorname{span}\left\{e_{1}, \ldots, e_{n}\right\}$ be the subspace with the norm inherited from $H$ and let $P_{n}: H \rightarrow H_{n}$ be defined by Eq. 20. Let us fix $m>\frac{d}{2}+1$ and let $U$ be the space defined by Eq. 16. Consider the following mapping

$$
B_{n}(u):=P_{n} B\left(\chi_{n}(u), u\right), \quad u \in H_{n},
$$

where $\chi_{n}: H \rightarrow H$ is defined by $\chi_{n}(u)=\theta_{n}\left(|u|_{U^{\prime}}\right) u$, where $\theta_{n}: \mathbb{R} \rightarrow[0,1]$ of class $\mathscr{C}^{\infty}$ such that

$$
\theta_{n}(r)=1 \quad \text { if } \quad r \leq n \quad \text { and } \quad \theta_{n}(r)=0 \quad \text { if } \quad r \geq n+1 .
$$

Since $H_{n} \subset H, B_{n}$ is well defined. Moreover, $B_{n}: H_{n} \rightarrow H_{n}$ is globally Lipschitz continuous. 
Let us consider the classical Faedo-Galerkin approximation in the space $H_{n}$

$$
\begin{aligned}
u_{n}(t) & =P_{n} u_{0}-\int_{0}^{t}\left[P_{n} \mathscr{A} u_{n}(s)+B_{n}\left(u_{n}(s)\right)-P_{n} f(s)\right] d s \\
& +\int_{0}^{t} \int_{Y} P_{n} F\left(s, u_{n}\left(s^{-}\right), y\right) \tilde{\eta}(d s, d y) \\
& +\int_{0}^{t} P_{n} G\left(s, u_{n}(s)\right) d W(s), \quad t \in[0, T] .
\end{aligned}
$$

Lemma 3 For each $n \in \mathbb{N}$, there exists a unique $\mathbb{F}$-adapted, càdlàg $H_{n}$ valued process $u_{n}$ satisfying the Galerkin (38).

Proof The assertion follows from Theorem 9.1 in [17].

Using the Itô formula, see [17] or [21], and the Burkholder-Davis-Gundy inequality, see [26], we will prove the following lemma about a priori estimates of the solutions $u_{n}$ of Eq. 38. In fact, these estimates hold provided the noise terms satisfy only condition (32) in assumption (F.2) and condition (35) in assumption (G.2).

Lemma 4 The processes $\left(u_{n}\right)_{n \in \mathbb{N}}$ satisfy the following estimates.

(i) For every $p \in[1,4+\gamma]$ there exists a positive constant $C_{1}(p)$ such that

$$
\sup _{n \geq 1} \mathbb{E}\left(\sup _{0 \leq s \leq T}\left|u_{n}(s)\right|_{H}^{p}\right) \leq C_{1}(p)
$$

(ii) There exists a positive constant $C_{2}$ such that

$$
\sup _{n \geq 1} \mathbb{E}\left[\int_{0}^{T}\left\|u_{n}(s)\right\|_{V}^{2} d s\right] \leq C_{2} .
$$

Let us recall that $\gamma>0$ is defined in assumption (F.2).

Proof For all $n \in \mathbb{N}$ and all $R>0$ let us define

$$
\tau_{n}(R):=\inf \left\{t \geq 0:\left|u_{n}(t)\right|_{H} \geq R\right\} \wedge T
$$

Since the process $\left(u_{n}(t)\right)_{t \in[0, T]}$ is $\mathbb{F}$-adapted and right-continuous, $\tau_{n}(R)$ is a stopping time. Moreover, since the process $\left(u_{n}\right)$ is càdlàg on $[0, T]$, the trajectories $t \mapsto u_{n}(t)$ are bounded on $[0, T], \mathbb{P}$-a.s. Thus $\tau_{n}(R) \uparrow T, \mathbb{P}$-a.s., as $R \uparrow \infty$. 
Assume first that $p=2$ or $p=4+\gamma$. Using the Itô formula to the function $\phi(x):=|x|^{p}:=|x|_{H}^{p}, x \in H$, we obtain for all $t \in[0, T]$

$$
\begin{aligned}
& \left|u_{n}\left(t \wedge \tau_{n}(R)\right)\right|^{p}=\left|P_{n} u_{0}\right|^{p} \\
& +\int_{0}^{t \wedge \tau_{n}(R)}\left\{p\left|u_{n}(s)\right|^{p-2}\left\langle u_{n}(s)-P_{n} \mathscr{A} u_{n}(s)-B_{n}\left(u_{n}(s)\right)+P_{n} f(s)\right\rangle\right\} d s \\
& +\int_{0}^{t \wedge \tau_{n}(R)} \int_{Y}\left\{\phi\left(u_{n}\left(s^{-}\right)+P_{n} F\left(s, u_{n}\left(s^{-}\right) ; y\right)\right)-\phi\left(u_{n}\left(s^{-}\right)\right)\right\} \tilde{\eta}(d s, d y) \\
& +\int_{0}^{t \wedge \tau_{n}(R)} \int_{Y}\left\{\phi\left(u_{n}\left(s^{-}\right)+P_{n} F\left(s, u_{n}\left(s^{-}\right) ; y\right)\right)-\phi\left(u_{n}\left(s^{-}\right)\right)\right. \\
& +\frac{1}{2} \int_{0}^{t \wedge \tau_{n}(R)} \operatorname{Tr}\left[P_{n} G\left(s, u_{n}(s)\right) \frac{\partial^{2} \phi}{\partial x^{2}}\left(P_{n} G\left(s, u_{n}(s)\right)\right)^{*}\right] d s \\
& +\int_{0}^{t \wedge \tau_{n}(R)} p\left|u_{n}(s)\right|^{p-2}\left\langle u_{n}(s) P_{n} G\left(s, u_{n}(s)\right) d W(s)\right\rangle .
\end{aligned}
$$

By Eqs. 11 and 15 we obtain for all $t \in[0, T]$

$$
\begin{aligned}
& \left|u_{n}\left(t \wedge \tau_{n}(R)\right)\right|^{p}=\left|P_{n} u_{0}\right|^{p} \\
& +\int_{0}^{t \wedge \tau_{n}(R)}\left\{-p\left|u_{n}(s)\right|^{p-2}\left\|u_{n}(s)\right\|^{2}+p\left|u_{n}(s)\right|^{p-2}\left\langle u_{n}(s) f(s)\right\rangle\right\} d s \\
& +\int_{0}^{t \wedge \tau_{n}(R)} \int_{Y}\left\{\left|u_{n}\left(s^{-}\right)+P_{n} F\left(s, u_{n}\left(s^{-}\right) ; y\right)\right|^{p}-\left|u_{n}\left(s^{-}\right)\right|^{p}\right\} \tilde{\eta}(d s, d y) \\
& +\int_{0}^{t \wedge \tau_{n}(R)} \int_{Y}\left\{\left|u_{n}\left(s^{-}\right)+P_{n} F\left(s, u_{n}\left(s^{-}\right) ; y\right)\right|^{p}-\left|u_{n}\left(s^{-}\right)\right|^{p}\right. \\
& +\frac{1}{2} \int_{0}^{t \wedge \tau_{n}(R)} \operatorname{Tr}\left[P_{n} G\left(s, u_{n}(s)\right) \frac{\partial^{2} \phi}{\partial x^{2}}\left(P_{n} G\left(s, u_{n}(s)\right)\right)^{*}\right] d s \\
& +\int_{0}^{t \wedge \tau_{n}(R)} p\left|u_{n}(s)\right|^{p-2}\left\langle u_{n}(s) G\left(s, u_{n}(s)\right) d W(s)\right\rangle .
\end{aligned}
$$

Let us recall that according to Eq. 15 we have $\langle\mathscr{A} u \mid u\rangle=((u \mid u))$ and thus

$$
2\langle\mathscr{A} u \mid u\rangle-a\|u\|^{2}=(2-a)\|u\|^{2} .
$$

Hence inequality (35) in assumption (G.2) can be written equivalently in the following form

$$
\|G(s, u)\|_{\mathscr{L}_{\mathrm{HS}}\left(Y_{W}, H\right)}^{2} \leq(2-a)\|u\|^{2}+\lambda|u|_{H}^{2}+\kappa, \quad u \in V, \quad s \in[0, T] .
$$


Hence

$$
\begin{aligned}
& \frac{1}{2} \int_{0}^{t \wedge \tau_{n}(R)} \operatorname{Tr}\left[P_{n} G\left(s, u_{n}(s)\right) \frac{\partial^{2} \phi}{\partial x^{2}}\left(P_{n} G\left(s, u_{n}(s)\right)\right)^{*}\right] d s \\
& \leq \frac{p(p-1)}{2} \int_{0}^{t \wedge \tau_{n}(R)}\left|u_{n}(s)\right|^{p-2}\left[(2-a)\left\|u_{n}(s)\right\|^{2}+\lambda\left|u_{n}(s)\right|^{2}+\kappa\right] d s .
\end{aligned}
$$

Moreover, by assumption (A.1), Eq. 8 and the Schwarz inequality, we obtain for every $\varepsilon>0$ and for all $s \in[0, T]$

$$
\begin{aligned}
& \left\langle f(s) \mid u_{n}(s)\right\rangle \leq|f(s)|_{V^{\prime}} \cdot\left\|u_{n}(s)\right\|_{V} \\
& \leq|f(s)|_{V^{\prime}}\left|u_{n}(s)\right|+\frac{1}{4 \varepsilon}|f(s)|_{V^{\prime}}^{2}+\varepsilon\left\|u_{n}(s)\right\|^{2}
\end{aligned}
$$

and hence by the Young inequality ${ }^{2}$

$$
\begin{aligned}
& \left|P_{n} u_{0}\right|^{p}+p\left|u_{n}(s)\right|^{p-2}\left(|f(s)|_{V^{\prime}}\left|u_{n}(s)\right|+\frac{1}{4 \varepsilon}|f(s)|_{V^{\prime}}^{2}\right) \\
& +\frac{p(p-1)}{2}\left|u_{n}(s)\right|^{p-2}\left[\lambda\left|u_{n}(s)\right|^{2}+\kappa\right]=\frac{p(p-1) \lambda}{2}\left|u_{n}(s)\right|^{p} \\
& +\left|P_{n} u_{0}\right|^{p}+p|f(s)|_{V^{\prime}}\left|u_{n}(s)\right|^{p-1}+p\left(\frac{1}{4 \varepsilon}|f(s)|_{V^{\prime}}^{2}+\frac{(p-1) \kappa}{2}\right)\left|u_{n}(s)\right|^{p-2} \\
& \leq c+c_{1}\left|u_{n}(s)\right|^{p}
\end{aligned}
$$

for some constants $c, c_{1}>0$. Thus

$$
\begin{aligned}
& \left|u_{n}\left(t \wedge \tau_{n}(R)\right)\right|^{p}+\left[p-p \varepsilon-\frac{1}{2} p(p-1)(2-a)\right] \int_{0}^{t \wedge \tau_{n}(R)}\left|u_{n}(s)\right|^{p-2}\left\|u_{n}(s)\right\|^{2} d s \\
& \leq c+c_{1} \int_{0}^{t \wedge \tau_{n}(R)}\left|u_{n}(s)\right|^{p} d s \\
& \quad+\int_{0}^{t \wedge \tau_{n}(R)} \int_{Y}\left\{\left|u_{n}\left(s^{-}\right)+P_{n} F\left(s, u_{n}\left(s^{-}\right) ; y\right)\right|^{p}-\left|u_{n}\left(s^{-}\right)\right|^{p}\right\} \tilde{\eta}(d s, d y) \\
& \quad+\int_{0}^{t \wedge \tau_{n}(R)} \int_{Y}\left\{\left|u_{n}\left(s^{-}\right)+P_{n} F\left(s, u_{n}\left(s^{-}\right) ; y\right)\right|^{p}-\left|u_{n}\left(s^{-}\right)\right|^{p}\right. \\
& \quad-\int_{0}^{t \wedge \tau_{n}(R)} p\left|u_{n}\left(s^{-}\right)\right|^{p-2}\left(u_{n}\left(s^{-}\right) \mid P_{n} F\left(s, u_{n}\left(s^{-}\right) ; y\right)_{H}\right\} v(d y) d s
\end{aligned}
$$

Let us choose $\varepsilon>0$ such that $p-p \varepsilon-\frac{1}{2} p(p-1)(2-a)>0$, or equivalently,

$$
\varepsilon<1-\frac{1}{2}(p-1)(2-a) .
$$

Note that since by assumption (G.2) $a \in\left(2-\frac{2}{3+\gamma}, 2\right]$, such an $\varepsilon$ exists.

$\overline{{ }^{2} a b \leq \frac{1}{q_{1}} a^{q_{1}}+\frac{1}{q_{2}} b^{q_{2}} \text { if } a, b>0}, q_{1}, q_{2} \in(1, \infty)$ and $\frac{1}{q_{1}}+\frac{1}{q_{2}}=1$. 
From the Taylor formula, it follows that for each $p \geq 2$ there exists a positive constant $c_{p}>0$ such that for all $x, h \in H$ the following inequality holds

$$
|| x+\left.h\right|_{H} ^{p}-|x|_{H}^{p}-\left.p|x|_{H}^{p-2}(x \mid h)_{H}\left|\leq c_{p}\left(|x|_{H}^{p-2}+|h|_{H}^{p-2}\right)\right| h\right|_{H} ^{2} .
$$

By Eqs. 43, 32 and 41, the process $\left(M_{n}\left(t \wedge \tau_{n}(R)\right)\right)_{t \in[0, T]}$, where

$$
M_{n}(t):=\int_{0}^{t} \int_{Y}\left\{\left|u_{n}\left(s^{-}\right)+P_{n} F\left(s, u_{n}\left(s^{-}\right) ; y\right)\right|^{p}-\left|u_{n}\left(s^{-}\right)\right|^{p}\right\} \tilde{\eta}(d s, d y),
$$

$t \in[0, T]$, is an integrable martingale. Hence $\mathbb{E}\left[M_{n}\left(t \wedge \tau_{n}(R)\right)\right]=0$ for all $t \in[0, T]$. By Eq. 35 and 41, the process $\left(N_{n}\left(t \wedge \tau_{n}(R)\right)\right)_{t \in[0, T]}$, where

$$
N_{n}(t):=\int_{0}^{t}\left|u_{n}(s)\right|^{p-2}\left\langle u_{n}(s) \mid G\left(s, u_{n}(s)\right) d W(s)\right\rangle, \quad t \in[0, T]
$$

is an integrable martingale and thus $\mathbb{E}\left[N_{n}\left(t \wedge \tau_{n}(R)\right)\right]=0$ for all $t \in[0, T]$.

Let us denote

$$
\begin{aligned}
& I_{n}(t):=\int_{0}^{t} \int_{Y}\left\{\left|u_{n}\left(s^{-}\right)+P_{n} F\left(s, u_{n}\left(s^{-}\right) ; y\right)\right|^{p}-\left|u_{n}\left(s^{-}\right)\right|^{p}\right. \\
& \left.-p\left|u_{n}\left(s^{-}\right)\right|^{p-2}\left(u_{n}\left(s^{-}\right) \mid P_{n} F\left(s, u_{n}\left(s^{-}\right) ; y\right)\right)_{H}\right\} v(d y) d s, \quad t \in[0, T] .
\end{aligned}
$$

By Eqs. 43 and 32 we obtain the following inequalities

$$
\begin{aligned}
& \left|I_{n}(t)\right| \\
& \leq c_{p} \int_{0}^{t} \int_{Y}\left|P_{n} F\left(s, u_{n}\left(s^{-}\right) ; y\right)\right|_{H}^{2}\left\{\left|u_{n}\left(s^{-}\right)\right|_{H}^{p-2}+\left|P_{n} F\left(s, u_{n}\left(s^{-}\right) ; y\right)\right|_{H}^{p-2}\right\} v(d y) d s \\
& \leq c_{p} \int_{0}^{t}\left\{C_{2}\left|u_{n}(s)\right|_{H}^{p-2}\left(1+\left|u_{n}(s)\right|_{H}^{2}\right)+C_{p}\left(1+\left|u_{n}(s)\right|_{H}^{p}\right)\right\} d s \\
& \leq \tilde{c}_{p} \int_{0}^{t}\left\{1+\left|u_{n}(s)\right|_{H}^{p}\right\} d s=\tilde{c}_{p} t+\tilde{c}_{p} \int_{0}^{t}\left|u_{n}(s)\right|_{H}^{p} d s, \quad t \in[0, T]
\end{aligned}
$$

for some constant $\tilde{c}_{p}>0$. Thus by the Fubini Theorem, we obtain the following inequality

$$
\mathbb{E}\left[\left|I_{n}(t)\right|\right] \leq \tilde{c}_{p} t+\tilde{c}_{p} \int_{0}^{t} \mathbb{E}\left[\left|u_{n}(s)\right|_{H}^{p}\right] d s, \quad t \in[0, T] .
$$

By Eqs. 42 and 45, we have for all $t \in[0, T]$

$$
\begin{aligned}
& \mathbb{E}\left[\left|u_{n}\left(t \wedge \tau_{n}(R)\right)\right|_{H}^{p}\right] \\
& +\left[p-p \varepsilon-\frac{1}{2} p(p-1)(2-a)\right] \mathbb{E}\left[\int_{0}^{T \wedge \tau_{n}(R)}\left|u_{n}(s)\right|_{H}^{p-2}\left\|u_{n}(s)\right\|^{2} d s\right] \\
& \leq c+\tilde{c}_{p} T+\left(c_{1}+\tilde{c}_{p}\right) \int_{0}^{t \wedge \tau_{n}(R)} \mathbb{E}\left[\left|u_{n}(s)\right|_{H}^{p}\right] d s .
\end{aligned}
$$

In particular,

$$
\mathbb{E}\left[\left|u_{n}\left(t \wedge \tau_{n}(R)\right)\right|_{H}^{p}\right] \leq c+\tilde{c}_{p} T+\left(c_{1}+\tilde{c}_{p}\right) \int_{0}^{t \wedge \tau_{n}(R)} \mathbb{E}\left[\left|u_{n}(s)\right|_{H}^{p}\right] d s .
$$


By the Gronwall Lemma we infer that for all $t \in[0, T]: \mathbb{E}\left[\left|u_{n}\left(t \wedge \tau_{n}(R)\right)\right|^{p}\right] \leq \tilde{\tilde{C}}_{p}$ for some constant $\tilde{\tilde{C}}_{p}$ independent of $t \in[0, T], R>0$ and $n \in \mathbb{N}$, i.e.

$$
\sup _{n \geq 1} \sup _{t \in\left[0, T \wedge \tau_{n}(R)\right]} \mathbb{E}\left[\left|u_{n}(t)\right|_{H}^{p}\right] \leq \tilde{\tilde{C}}_{p}
$$

Hence, in particular,

$$
\sup _{n \geq 1} \mathbb{E}\left[\int_{0}^{T \wedge \tau_{n}(R)}\left|u_{n}(s)\right|_{H}^{p} d s\right] \leq \tilde{C}_{p}
$$

for some constant $\tilde{C}_{p}>0$. Passing to the limit as $R \uparrow \infty$, by the Fatou Lemma we infer that

$$
\sup _{n \geq 1} \mathbb{E}\left[\int_{0}^{T}\left|u_{n}(s)\right|_{H}^{p} d s\right] \leq \tilde{C}_{p}
$$

By Eqs. 46 and 47, we infer that

$$
\sup _{n \geq 1} \mathbb{E}\left[\int_{0}^{T \wedge \tau_{n}(R)}\left|u_{n}(s)\right|_{H}^{p-2}\left\|u_{n}(s)\right\|^{2} d s\right] \leq C_{p}
$$

for some positive constant $C_{p}$. Passing to the limit as $R \uparrow \infty$ and using again the Fatou Lemma we infer that

$$
\sup _{n \geq 1} \mathbb{E}\left[\int_{0}^{T}\left|u_{n}(s)\right|_{H}^{p-2}\left\|u_{n}(s)\right\|^{2} d s\right] \leq C_{p} .
$$

In particular, putting $p:=2$ by Eqs. 8, 49 and 47 we obtain assertion (40).

Let us move to the proof of inequality (39). By the Burkholder-Davis-Gundy inequality we obtain

$$
\begin{aligned}
& \mathbb{E}\left[\sup _{r \in[0, t]}\left|M_{n}\left(r \wedge \tau_{n}(R)\right)\right|\right] \\
& =\mathbb{E}\left[\sup _{r \in[0, t]}\left|\int_{0}^{r \wedge \tau_{n}(R)} \int_{Y}\left\{\left|u_{n}\left(s^{-}\right)+P_{n} F\left(s, u_{n}\left(s^{-}\right) ; y\right)\right|_{H}^{p}-\left|u_{n}\left(s^{-}\right)\right|_{H}^{p}\right\} \tilde{\eta}(d s, d y)\right|\right] \\
& \leq \tilde{K}_{p} \mathbb{E}\left[\left(\int_{0}^{t \wedge \tau_{n}(R)} \int_{Y}\left(\left|u_{n}\left(s^{-}\right)+P_{n} F\left(s, u_{n}\left(s^{-}\right) ; y\right)\right|_{H}^{p}-\left|u_{n}\left(s^{-}\right)\right|_{H}^{p}\right)^{2} v(d y) d s\right)^{\frac{1}{2}}\right]
\end{aligned}
$$

for some constant $\tilde{K}_{p}>0$. By Eq. 43 and the Schwarz inequality we obtain the following inequalities for all $x, h \in H$

$$
\begin{aligned}
& \left(|x+h|_{H}^{p}-|x|_{H}^{p}\right)^{2} \leq 2\left\{p^{2}|x|_{H}^{2 p-2}|h|_{H}^{2}+c_{p}^{2}\left(|x|_{H}^{p-2}+|h|_{H}^{p-2}\right)^{2}|h|_{H}^{4}\right\} \\
& \leq 2 p^{2}|x|_{H}^{2 p-2}|h|_{H}^{2}+4 c_{p}^{2}|x|_{H}^{2 p-4}|h|_{H}^{4}+4 c_{p}^{2}|h|_{H}^{2 p} .
\end{aligned}
$$


Hence by inequality (32) in assumption (F.2) we obtain for all $s \in[0, T]$

$$
\begin{aligned}
& \int_{Y}\left(\left|u_{n}\left(s^{-}\right)+P_{n} F\left(s, u_{n}\left(s^{-}\right) ; y\right)\right|_{H}^{p}-\left|u_{n}\left(s^{-}\right)\right|_{H}^{p}\right)^{2} v(d y) \\
& \leq 2 p^{2}\left|u_{n}\left(s^{-}\right)\right|_{H}^{2 p-2} \int_{Y}\left|F\left(s, u_{n}\left(s^{-}\right) ; y\right)\right|_{H}^{2} v(d y) \\
& +4 c_{p}^{2}\left|u_{n}\left(s^{-}\right)\right|_{H}^{2 p-4} \int_{Y}\left|F\left(s, u_{n}\left(s^{-}\right) ; y\right)\right|_{H}^{4} v(d y)+4 c_{p}^{2} \int_{Y}\left|F\left(s, u_{n}\left(s^{-}\right) ; y\right)\right|_{H}^{2 p} v(d y) \\
& \leq C_{1}+C_{2}\left|u_{n}\left(s^{-}\right)\right|_{H}^{2 p-4}+C_{3}\left|u_{n}\left(s^{-}\right)\right|_{H}^{2 p-2}+C_{4}\left|u_{n}\left(s^{-}\right)\right|_{H}^{2 p}
\end{aligned}
$$

for some positive constants $C_{i}, i=1, \ldots, 4$. By Eq. 51 and the Young inequality we infer that

$$
\int_{Y}\left(\left|u_{n}\left(s^{-}\right)+P_{n} F\left(s, u_{n}\left(s^{-}\right) ; y\right)\right|_{H}^{p}-\left|u_{n}\left(s^{-}\right)\right|_{H}^{p}\right)^{2} v(d y) \leq K_{1}+K_{2}\left|u_{n}\left(s^{-}\right)\right|_{H}^{2 p}
$$

for some positive constants $K_{1}$ and $K_{2}$. Thus

$$
\begin{aligned}
& \left(\int_{0}^{t \wedge \tau_{n}(R)} \int_{Y}\left(\left|u_{n}\left(s^{-}\right)+P_{n} F\left(s, u_{n}\left(s^{-}\right) ; y\right)\right|_{H}^{p}-\left|u_{n}\left(s^{-}\right)\right|_{H}^{p}\right)^{2} v(d y) d s\right)^{\frac{1}{2}} \\
& \leq \sqrt{T K_{1}}+\sqrt{K_{2}}\left(\int_{0}^{t \wedge \tau_{n}(R)}\left|u_{n}(s)\right|_{H}^{2 p} d s\right)^{\frac{1}{2}} .
\end{aligned}
$$

By Eqs. 50, 52 and 47 we obtain the following inequalities

$$
\begin{aligned}
& \mathbb{E}\left[\sup _{r \in[0, t]}\left|M_{n}\left(r \wedge \tau_{n}(R)\right)\right|\right] \\
& \leq \tilde{K}_{p} \sqrt{T K_{1}}+\tilde{K}_{p} \sqrt{K_{2}} \mathbb{E}\left[\left(\int_{0}^{t \wedge \tau_{n}(R)}\left|u_{n}(s)\right|_{H}^{2 p} d s\right)^{\frac{1}{2}}\right] \\
& \leq \tilde{K}_{p} \sqrt{T K_{1}}+\tilde{K}_{p} \sqrt{K_{2}} \mathbb{E}\left[\left(\sup _{s \in[0, t]}\left|u_{n}\left(s \wedge \tau_{n}(R)\right)\right|_{H}^{p}\right)^{\frac{1}{2}}\left(\int_{0}^{t \wedge \tau_{n}(R)}\left|u_{n}(s)\right|_{H}^{p} d s\right)^{\frac{1}{2}}\right] \\
& \leq \tilde{K}_{p} \sqrt{T K_{1}}+\frac{1}{4} \mathbb{E}\left[\sup _{s \in[0, t]}\left|u_{n}\left(s \wedge \tau_{n}(R)\right)\right|_{H}^{p}\right]+\tilde{K}_{p}^{2} K_{2} \mathbb{E}\left[\int_{0}^{t \wedge \tau_{n}(R)}\left|u_{n}(s)\right|_{H}^{p} d s\right] \\
& \leq \frac{1}{4} \mathbb{E}\left[\sup _{s \in[0, t]}\left|u_{n}\left(s \wedge \tau_{n}(R)\right)\right|_{H}^{p}\right]+\tilde{K},
\end{aligned}
$$

where $\tilde{K}=\tilde{K}_{p} \sqrt{T K_{1}}+\tilde{K}_{p}^{2} K_{2} \tilde{C}_{p}$. (The constant $\tilde{C}_{p}$ is the same as in Eq. 47 ). 
Similarly, by the Burkholder-Davis-Gundy inequality we obtain

$$
\begin{aligned}
& \mathbb{E}\left[\sup _{r \in\left[0, t \wedge \tau_{n}(R)\right]}\left|N_{n}(r)\right|\right] \\
& =\mathbb{E}\left[\left.\sup _{r \in\left[0, t \wedge \tau_{n}(R)\right]}\left|\int_{0}^{r} p\right| u_{n}(s)\right|^{p-2}\left\langle u_{n}(s) P_{n} G\left(s, u_{n}(s)\right) d W(s)\right\rangle \mid\right] \\
& \leq C p \cdot \mathbb{E}\left[\left(\int_{0}^{t \wedge \tau_{n}(R)}\left|u_{n}(s)\right|^{2 p-2} \cdot \| G\left(s, u_{n}(s)\right)_{\mathscr{L}_{\mathrm{HS}}(Y, H)}^{2} d s\right)^{\frac{1}{2}}\right] \\
& \leq C p \mathbb{E}\left[\left(\sup _{s \in\left[0, t \wedge \tau_{n}(R)\right]}\left|u_{n}(s)\right|^{p}\right)^{\frac{1}{2}}\left(\int_{0}^{t \wedge \tau_{n}(R)}\left|u_{n}(s)\right|^{p-2} \cdot\left\|G\left(s, u_{n}(s)\right)\right\|_{\mathscr{L}_{\mathrm{HS}}(Y, H)}^{2} d s\right)^{\frac{1}{2}}\right] \text {. }
\end{aligned}
$$

By inequality (35) in assumption (G.2) and estimates (49), (47) we have the following inequalities

$$
\begin{aligned}
& \mathbb{E}\left[\sup _{r \in[0, t]}\left|N_{n}\left(r \wedge \tau_{n}(R)\right)\right|\right] \leq p \cdot \mathbb{E}\left[\left(\sup _{s \in[0, t]}\left|u_{n}\left(s \wedge \tau_{n}(R)\right)\right|^{p}\right)^{\frac{1}{2}}\right. \\
& \left.\cdot\left(\int_{0}^{t \wedge \tau_{n}(R)}\left|u_{n}(s)\right|^{p-2} \cdot\left[\lambda\left|u_{n}(s)\right|^{2}+\kappa+(2-a) \| u_{n}(s) 2\right] d s\right)^{\frac{1}{2}}\right] \\
& \leq \frac{1}{4} \mathbb{E}\left[\sup _{r \in[0, t]}\left|u_{n}\left(r \wedge \tau_{n}(R)\right)\right|^{p}\right] \\
& +C^{2} p^{2} \mathbb{E}\left[\int_{0}^{t \wedge \tau_{n}(R)}\left[\lambda\left|u_{n}(s)\right|^{p}+\kappa\left|u_{n}(s)\right|^{p-2}+(2-a)\left|u_{n}(s)\right|^{p-2} \| u_{n}(s) 2\right] d s\right] \\
& \leq \frac{1}{4} \mathbb{E}\left[\sup _{r \in[0, t]}\left|u_{n}\left(r \wedge \tau_{n}(R)\right)\right|^{p}\right]+\tilde{\tilde{K}}
\end{aligned}
$$

where $\tilde{\tilde{K}}=C^{2} p^{2}\left[\lambda \tilde{C}_{p}+\kappa \tilde{C}_{p-2}+(2-a) C_{2}\right]$. (The constants $\tilde{C}_{p}, \tilde{C}_{p-2}$ are the same as in Eq. 47 and $C_{2}$ is the same as in Eq. 49.) Therefore by Eq. 42 for all $t \in[0, T]$

$$
\begin{aligned}
& \left|u_{n}\left(t \wedge \tau_{n}(R)\right)\right|^{p} \leq c+c_{1} \int_{0}^{T}\left|u_{n}(s)\right|^{p} d s+\sup _{r \in[0, T]}\left|M_{n}\left(r \wedge \tau_{n}(R)\right)\right| \\
& +\left|I_{n}\left(T \wedge \tau_{n}(R)\right)\right|+\sup _{r \in[0, T]}\left|N_{n}\left(r \wedge \tau_{n}(R)\right)\right|,
\end{aligned}
$$


where $I_{n}$ is defined by Eq. 44. Since inequality (55) holds for all $t \in[0, T]$ and the right-hand side of Eq. 55 is independent of $t$, we infer that

$$
\begin{aligned}
& \mathbb{E}\left[\sup _{t \in T \wedge \tau_{n}(R)}\left|u_{n}(t)\right|^{p}\right] \leq c+c_{1} \mathbb{E}\left[\int_{0}^{T}\left|u_{n}(s)\right|^{p} d s\right] \\
& +\mathbb{E}\left[\sup _{r \in\left[0, T \wedge \tau_{n}(R)\right]}\left|M_{n}(r)\right|\right]+\mathbb{E}\left[\left|I_{n}\left(T \wedge \tau_{n}(R)\right)\right|\right]+\mathbb{E}\left[\sup _{r \in\left[0, T \wedge \tau_{n}(R)\right]}\left|N_{n}(r)\right|\right] .
\end{aligned}
$$

Using inequalities (47), (53), (45) and (54) in Eq. 56 we infer that

$$
\mathbb{E}\left[\sup _{t \in T \wedge \tau_{n}(R)}\left|u_{n}(t)\right|^{p}\right] \leq C_{1}(p)
$$

for some constant $C_{1}(p)$ independent of $n \in \mathbb{N}$ and $R>0$. Passing to the limit as $R \rightarrow \infty$, we obtain inequality (39). Thus the Lemma holds for $p \in\{2,4+\gamma\}$.

Let now $p \in[1,4+\gamma) \backslash\{2\}$. Let us fix $n \in \mathbb{N}$. Then

$$
\left|u_{n}(t)\right|_{H}^{p}=\left(\left|u_{n}(t)\right|_{H}^{4+\gamma}\right)^{\frac{p}{4+\gamma}} \leq\left(\sup _{t \in[0, T]}\left|u_{n}(t)\right|_{H}^{4+\gamma}\right)^{\frac{p}{4+\gamma}}, \quad t \in[0, T] .
$$

Thus

$$
\sup _{t \in[0, T]}\left|u_{n}(t)\right|_{H}^{p} \leq\left(\sup _{t \in[0, T]}\left|u_{n}(t)\right|_{H}^{4+\gamma}\right)^{\frac{p}{4+\gamma}}
$$

and by the Hólder inequality

$$
\begin{aligned}
\mathbb{E}\left[\sup _{t \in[0, T]}\left|u_{n}(t)\right|_{H}^{p}\right] & \leq \mathbb{E}\left[\left(\sup _{t \in[0, T]}\left|u_{n}(t)\right|_{H}^{4+\gamma}\right)^{\frac{p}{4+\gamma}}\right] \\
& \leq\left(\mathbb{E}\left[\sup _{t \in[0, T]}\left|u_{n}(t)\right|_{H}^{4+\gamma}\right]\right)^{\frac{p}{4+\gamma}} \leq\left[C_{1}(4+\gamma)\right]^{\frac{p}{4+\gamma}} .
\end{aligned}
$$

Since $n \in \mathbb{N}$ was chosen in an arbitray way, we infer that

$$
\sup _{n \in \mathbb{N}} \mathbb{E}\left[\sup _{t \in[0, T]}\left|u_{n}(t)\right|_{H}^{p}\right] \leq C_{1}(p)
$$

where $C_{1}(p)=\left[C_{1}(4+\gamma)\right]^{\frac{p}{4+\gamma}}$. The proof of Lemma is thus complete.

\subsection{Tightness}

Let $m>\frac{d}{2}+1$ be fixed and let $U$ be the space defined by Eq. 16 . We will apply Corollary 1 with $q:=2$. So, let us consider the space

$$
\mathscr{Z}:=L_{w}^{2}(0, T ; V) \cap L^{2}\left(0, T ; H_{\text {loc }}\right) \cap \mathbb{D}\left([0, T] ; U^{\prime}\right) \cap \mathbb{D}\left([0, T] ; H_{w}\right) .
$$


For each $n \in \mathbb{N}$, the solution $u_{n}$ of the Galerkin equation defines a measure $\mathscr{L}\left(u_{n}\right)$ on $(\mathscr{Z}, \mathscr{T})$. Using Corollary 1 we will prove that the set of measures $\left\{\mathscr{L}\left(u_{n}\right), n \in \mathbb{N}\right\}$ is tight on $(\mathscr{Z}, \mathscr{T})$. The inequalities (39) and (40) in Lemma 4 are of crucial importance. However, to prove tightness it is sufficient to use inequality (39) only with $p=2$.

Lemma 5 The set of measures $\left\{\mathscr{L}\left(u_{n}\right), n \in \mathbb{N}\right\}$ is tight on $(\mathscr{Z}, \mathscr{T})$.

Proof We will apply Corollary 1. By estimates (39) and (40), conditions (a), (b) are satisfied. Thus, it is sufficient to prove that the sequence $\left(u_{n}\right)_{n \in \mathbb{N}}$ satisfies the Aldous condition [A] in the space $U^{\prime}$. We will use Lemma 9 in Appendix A. Let $\left(\tau_{n}\right)_{n \in \mathbb{N}}$ be a sequence of stopping times such that $0 \leq \tau_{n} \leq T$. By Eq. 38, we have

$$
\begin{aligned}
u_{n}(t)= & P_{n} u_{0}-\int_{0}^{t} P_{n} \mathscr{A} u_{n}(s) d s-\int_{0}^{t} B_{n}\left(u_{n}(s)\right) d s+\int_{0}^{t} P_{n} f(s) d s \\
& +\int_{0}^{t} \int_{Y} P_{n} F\left(s, u_{n}\left(s^{-}\right), y\right) \tilde{\eta}(d s, d y)+\int_{0}^{t} P_{n} G\left(s, u_{n}(s)\right) d W(s) \\
& =: J_{1}^{n}+J_{2}^{n}(t)+J_{3}^{n}(t)+J_{4}^{n}(t)+J_{5}^{n}(t)+J_{6}^{n}(t), \quad t \in[0, T]
\end{aligned}
$$

Let $\theta>0$. We will check that each term $J_{i}^{n}, \mathrm{i}=1, \ldots, 6$, satisfies condition (89) in Lemma 9.

Since $\mathscr{A}: V \rightarrow V^{\prime}$ and $|\mathscr{A}(u)|_{V^{\prime}} \leq \| u$ and the embedding $V^{\prime} \hookrightarrow U^{\prime}$ is continuous, by the Hólder inequality and Eq. 40, we have the following estimates

$$
\begin{aligned}
& \mathbb{E}\left[\left|J_{2}^{n}\left(\tau_{n}+\theta\right)-J_{2}^{n}\left(\tau_{n}\right)\right|_{U^{\prime}}\right]=\mathbb{E}\left[\left|\int_{\tau_{n}}^{\tau_{n}+\theta} P_{n} \mathscr{A} u_{n}(s) d s\right|_{U^{\prime}}\right] \\
& \leq c \mathbb{E}\left[\int_{\tau_{n}}^{\tau_{n}+\theta}\left|\mathscr{A} u_{n}(s)\right|_{V^{\prime}} d s\right] \leq c \mathbb{E}\left[\int_{\tau_{n}}^{\tau_{n}+\theta}\left\|u_{n}(s)\right\| d s\right] \\
& \leq c \mathbb{E}\left[\theta^{\frac{1}{2}}\left(\int_{0}^{T}\left\|u_{n}(s)\right\|^{2} d s\right)^{\frac{1}{2}}\right] \leq c \sqrt{C_{2}} \cdot \theta^{\frac{1}{2}}=: c_{2} \cdot \theta^{\frac{1}{2}}
\end{aligned}
$$

Thus $J_{2}^{n}$ satifies condition (89) with $\alpha=1$ and $\beta=\frac{1}{2}$.

Let us consider the term $J_{3}^{n}$. Since $m>\frac{d}{2}+1$ and $U \hookrightarrow V_{m}$, by Eqs. 12 and 39 we have the following inequalities

$$
\begin{aligned}
& \mathbb{E}\left[\left|J_{3}^{n}\left(\tau_{n}+\theta\right)-J_{3}^{n}\left(\tau_{n}\right)\right|_{U^{\prime}}\right]=\mathbb{E}\left[\left|\int_{\tau_{n}}^{\tau_{n}+\theta} B_{n}\left(u_{n}(s)\right) d s\right|_{U^{\prime}}\right] \\
& \leq c \mathbb{E}\left[\int_{\tau_{n}}^{\tau_{n}+\theta}\left|B\left(u_{n}(s)\right)\right|_{V_{m}^{\prime}} d s\right] \leq c \mathbb{E}\left[\int_{\tau_{n}}^{\tau_{n}+\theta}\|B\| \cdot\left|u_{n}(s)\right|_{H}^{2} d s\right] \\
& \leq c\|B\| \cdot \mathbb{E}\left[\sup _{s \in[0, T]}\left|u_{n}(s)\right|_{H}^{2}\right] \cdot \theta \leq c\|B\| C_{1}(2) \cdot \theta=: c_{3} \cdot \theta,
\end{aligned}
$$

where $\|B\|$ stands for the norm of $B: H \times H \rightarrow V_{m}^{\prime}$. This means that $J_{3}^{n}$ satisfies condition (89) with $\alpha=\beta=1$. 
Let us move to the term $J_{4}^{n}$. By the Hólder inequality, we have

$$
\begin{aligned}
& \mathbb{E}\left[\left|J_{4}^{n}\left(\tau_{n}+\theta\right)-J_{4}^{n}\left(\tau_{n}\right)\right|_{U^{\prime}}\right] \leq c \mathbb{E}\left[\left|\int_{\tau_{n}}^{\tau_{n}+\theta} P_{n} f(s) d s\right|_{V^{\prime}}\right] \\
& \leq c \cdot \theta^{\frac{1}{2}} \cdot \mathbb{E}\left[\left(\int_{0}^{T}|f(s)|_{V^{\prime}}^{2} d s\right)^{\frac{1}{2}}\right]=c \cdot \theta^{\frac{1}{2}} \cdot\|f\|_{L^{2}\left(0, T ; V^{\prime}\right)}=: c_{4} \cdot \theta^{\frac{1}{2}} .
\end{aligned}
$$

Hence condition (89) holds with $\alpha=1$ and $\beta=\frac{1}{2}$.

Let us consider the term $J_{5}^{n}$. Since $H \hookrightarrow U^{\prime}$, by Eq. 29, condition (32) with $p=2$ in Assumption (F.2) and by Eq. 39, we obtain the following inequalities

$$
\begin{aligned}
& \mathbb{E}\left[\left|J_{5}^{n}\left(\tau_{n}+\theta\right)-J_{5}^{n}\left(\tau_{n}\right)\right|_{U^{\prime}}^{2}\right]=\mathbb{E}\left[\left|\int_{\tau_{n}}^{\tau_{n}+\theta} \int_{Y} P_{n} F\left(s, u_{n}(s) ; y\right) \tilde{\eta}(d s, d y)\right|_{U^{\prime}}^{2}\right] \\
& \leq c \mathbb{E}\left[\left|\int_{\tau_{n}}^{\tau_{n}+\theta} \int_{Y} P_{n} F\left(s, u_{n}(s) ; y\right) \tilde{\eta}(d s, d y)\right|_{H}^{2}\right] \\
& =c \mathbb{E}\left[\int_{\tau_{n}}^{\tau_{n}+\theta} \int_{Y}\left|P_{n} F\left(s, u_{n}(s) ; y\right)\right|_{H}^{2} v(d y) d s\right] \leq C \mathbb{E}\left[\int_{\tau_{n}}^{\tau_{n}+\theta}\left(1+\left|u_{n}(s)\right|_{H}^{2}\right) d s\right] \\
& \leq C \cdot \theta \cdot\left(1+\mathbb{E}\left[\sup _{s \in[0, T]}\left|u_{n}(s)\right|_{H}^{2}\right]\right) \leq C \cdot\left(1+C_{1}(2)\right) \cdot \theta=: c_{5} \cdot \theta .
\end{aligned}
$$

Thus $J_{5}^{n}$ satisfies condition (89) with $\alpha=2$ and $\beta=1$.

Let us consider the term $J_{6}^{n}$. By the Itô isometry, condition (36) in assumption (G.3), continuity of the embedding $V^{\prime} \hookrightarrow U^{\prime}$ and inequality (39), we have

$$
\begin{aligned}
& \mathbb{E}\left[\left|J_{6}^{n}\left(\tau_{n}+\theta\right)-J_{6}^{n}\left(\tau_{n}\right)\right|_{U^{\prime}}^{2}\right]=\mathbb{E}\left[\left|\int_{\tau_{n}}^{\tau_{n}+\theta} P_{n} G\left(s, u_{n}(s)\right) d W(s)\right|_{U^{\prime}}^{2}\right] \\
& \leq c \mathbb{E}\left[\int_{\tau_{n}}^{\tau_{n}+\theta}\left(1+\left|u_{n}(s)\right|_{H}^{2}\right) d s\right] \leq c \theta\left(1+\mathbb{E}\left[\sup _{s \in[0, T]}\left|u_{n}(s)\right|_{H}^{2}\right]\right) \leq c\left(1+C_{1}(2)\right) \theta .
\end{aligned}
$$

Thus $J_{6}^{n}$ satisfies condition (89) with $\alpha=2$ and $\beta=1$.

By Lemma 9 the sequence $\left(u_{n}\right)_{n \in \mathbb{N}}$ satisfies the Aldous condition in the space $U^{\prime}$. This completes the proof of Lemma.

We will now move to the proof of the main Theorem of existence of a martingale solution. The main difficulties occur in the term containing the nonlinearity $B$ and in the noise terms $F$ and $G$. To deal with the nonlinear term, we need inequality (39) for some $p>4$. Moreover, we will see that the sequence $\left(\bar{u}_{n}\right)$ of approximate solutions is convergent in the Fréchet space $L^{2}\left(0, T ; H_{l o c}\right)$. So, we will use the property of the mapping $B$ contained in Lemma 6 below. Analogous problems appear in the noise terms, where assumptions (F.3) and (G.3) will be needed in the case when the domain $\mathscr{O}$ is unbounded. For simplicity we assume that $\operatorname{dim} Y_{W}=1$, i.e. we consider onedimensional cylindrical Wiener process $W(t), t \in[0, T]$. Construction of a martingale solution is based on the Skorokhod Theorem for nonmetric spaces. The method is closely related to the approach due to Brzeźniak and Hausenblas [6]. 


\subsection{Proof of Theorem 3}

By Lemma 5 the set of measures $\left\{\mathscr{L}\left(u_{n}\right), n \in \mathbb{N}\right\}$ is tight on the space $(\mathscr{Z}, \mathscr{T})$. Let $\eta_{n}:=\eta, n \in \mathbb{N}$. The set of measures $\left\{\mathscr{L}\left(\eta_{n}\right), n \in \mathbb{N}\right\}$ is tight on the space $M_{\overline{\mathbb{N}}}([0, T] \times$ $Y)$. Let $W_{n}:=W, n \in \mathbb{N}$. The set $\left\{\mathscr{L}\left(W_{n}\right), n \in \mathbb{N}\right\}$ is tight on the space $\mathscr{C}([0, T] ; \mathbb{R})$ of continuous function from $[0, T]$ to $\mathbb{R}$ with the standard supremum-norm. Thus the set $\left\{\mathscr{L}\left(u_{n}, \eta_{n}, W_{n}\right), n \in \mathbb{N}\right\}$ is tight on $\mathscr{Z} \times M_{\overline{\mathbb{N}}}([0, T] \times Y) \times \mathscr{C}([0, T] ; \mathbb{R})$. By Corollary 1 and Remark 2, see Appendix B, there exists a subsequence $\left(n_{k}\right)_{k \in \mathbb{N}}$, a probability space $(\bar{\Omega}, \overline{\mathscr{F}}, \overline{\mathbb{P}})$ and, on this space, $\mathscr{Z} \times M_{\overline{\mathbb{N}}}([0, T] \times Y) \times \mathscr{C}([0, T] ; \mathbb{R})$ valued random variables $\left(u_{*}, \eta_{*}, W_{*}\right),\left(\bar{u}_{k}, \bar{\eta}_{k}, \bar{W}_{k}\right), k \in \mathbb{N}$ such that

(i) $\mathscr{L}\left(\left(\bar{u}_{k}, \bar{\eta}_{k}, \bar{W}_{k}\right)\right)=\mathscr{L}\left(\left(u_{n_{k}}, \eta_{n_{k}}, W_{n_{k}}\right)\right)$ for all $k \in \mathbb{N}$;

(ii) $\quad\left(\bar{u}_{k}, \bar{\eta}_{k}, \bar{W}_{k}\right) \rightarrow\left(u_{*}, \eta_{*}, W_{*}\right)$ in $\mathscr{Z} \times M_{\overline{\mathbb{N}}}([0, T] \times Y) \times \mathscr{C}([0, T] ; \mathbb{R})$ with probability 1 on $(\bar{\Omega}, \overline{\mathscr{F}}, \overline{\mathbb{P}})$ as $k \rightarrow \infty$;

(iii) $\quad\left(\bar{\eta}_{k}(\bar{\omega}), \bar{W}_{k}(\bar{\omega})\right)=\left(\eta_{*}(\bar{\omega}), W_{*}(\bar{\omega})\right)$ for all $\bar{\omega} \in \bar{\Omega}$.

We will denote this sequences again by $\left(\left(u_{n}, \eta_{n}, W_{n}\right)\right)_{n \in \mathbb{N}}$ and $\left(\left(\bar{u}_{n}, \bar{\eta}_{n}, \bar{W}_{n}\right)\right)_{n \in \mathbb{N}}$. Moreover, $\bar{\eta}_{n}, n \in \mathbb{N}$, and $\eta_{*}$ are time homogeneous Poisson random measures on $(Y, \mathscr{Y})$ with intensity measure $v$ and $\bar{W}_{n}, n \in \mathbb{N}$, and $W_{*}$ are cylindrical Wiener processes, see [6, Section 9]. Using the definition of the space $\mathscr{Z}$, see Eq. 57, in particular, we have

$$
\bar{u}_{n} \rightarrow u_{*} \quad \text { in } \quad L_{w}^{2}(0, T ; V) \cap L^{2}\left(0, T ; H_{\mathrm{loc}}\right) \cap \mathbb{D}\left([0, T] ; U^{\prime}\right) \cap \mathbb{D}\left([0, T] ; H_{w}\right) \quad \overline{\mathbb{P}} \text {-a.s. }
$$

Since the random variables $\bar{u}_{n}$ and $u_{n}$ are identically distributed, we have the following inequalities. For every $p \in[1,4+\gamma]$

$$
\sup _{n \geq 1} \overline{\mathbb{E}}\left(\sup _{0 \leq s \leq T}\left|\bar{u}_{n}(s)\right|_{H}^{p}\right) \leq C_{1}(p) .
$$

and

$$
\sup _{n \geq 1} \overline{\mathbb{E}}\left[\int_{0}^{T}\left\|\bar{u}_{n}(s)\right\|_{V}^{2} d s\right] \leq C_{2} .
$$

Let us fix $v \in U$. Analogously to [6], let us denote

$$
\begin{aligned}
& \mathscr{K}_{n}\left(\bar{u}_{n}, \bar{\eta}_{n}, \bar{W}_{n}, v\right)(t):=\left(\bar{u}_{n}(0) \mid v\right)_{H} \\
& +\int_{0}^{t}\left\langle P_{n} \mathscr{A} \bar{u}_{n}(s) \mid v\right\rangle d s+\int_{0}^{t}\left\langle B_{n}\left(\bar{u}_{n}(s)\right) \mid v\right\rangle d s \\
& +\int_{0}^{t}\left\langle P_{n} f(s) \mid v\right\rangle d s+\int_{0}^{t} \int_{Y}\left(P_{n} F\left(s, \bar{u}_{n}\left(s^{-}\right) ; y\right)|v\rangle_{H} \tilde{\bar{\eta}}_{n}(d s, d y)\right. \\
& +\left\langle\int_{0}^{t} P_{n} G\left(s, \bar{u}_{n}(s)\right) d \bar{W}_{n}(s) \mid v\right\rangle
\end{aligned}
$$


and

$$
\begin{aligned}
& \mathscr{K}\left(u_{*}, \eta_{*}, W_{*}, v\right)(t):=\left(u_{*}(0) \mid v\right)_{H}+\int_{0}^{t}\left\langle\mathscr{A} u_{*}(s) \mid v\right\rangle d s+\int_{0}^{t}\left\langle B\left(u_{*}(s)\right) \mid v\right\rangle d s \\
& +\int_{0}^{t}\langle f(s) \mid v\rangle d s+\int_{0}^{t} \int_{Y}\left(F\left(s, u_{*}\left(s^{-}\right) ; y\right)|v\rangle_{H} \tilde{\eta}_{*}(d s, d y)\right. \\
& +\left\langle\int_{0}^{t} G\left(s, u_{*}(s)\right) d W_{*}(s) \mid v\right\rangle, \quad t \in[0, T] .
\end{aligned}
$$

Step $1^{0}$ We will prove that

$$
\lim _{n \rightarrow \infty}\left\|\left(\bar{u}_{n}(\cdot) \mid v\right)_{H}-\left(u_{*}(\cdot) \mid v\right)_{H}\right\|_{L^{2}([0, T] \times \bar{\Omega})}=0
$$

and

$$
\lim _{n \rightarrow \infty}\left\|\mathscr{K}_{n}\left(\bar{u}_{n}, \bar{\eta}_{n}, \bar{W}_{n}, v\right)-\mathscr{K}\left(u_{*}, \eta_{*}, W_{*}, v\right)\right\|_{L^{2}([0, T] \times \bar{\Omega})}=0
$$

To prove Eq. 63 let us write

$$
\begin{aligned}
& \left\|\left(\bar{u}_{n}(\cdot) \mid v\right)_{H}-\left(u_{*}(\cdot) \mid v\right)_{H}\right\|_{L^{2}([0, T] \times \bar{\Omega})}^{2} \\
& =\int_{\bar{\Omega}} \int_{0}^{T}\left|\left(\bar{u}_{n}(t)-u_{*}(t) \mid v\right)_{H}\right|^{2} d t \overline{\mathbb{P}}(d \omega)=\overline{\mathbb{E}}\left[\int_{0}^{T}\left|\left(\bar{u}_{n}(t)-u_{*}(t) \mid v\right)_{H}\right|^{2} d t\right] .
\end{aligned}
$$

Moreover,

$$
\begin{aligned}
& \int_{0}^{T}\left|\left(\bar{u}_{n}(t)-u_{*}(t) \mid v\right)_{H}\right|^{2} d t=\int_{0}^{T}\left|\left\langle\bar{u}_{n}(t)-u_{*}(t) \mid v\right\rangle_{U^{\prime}, U}\right|^{2} d t \\
& \leq\|v\|_{U}^{2} \int_{0}^{T}\left|\bar{u}_{n}(t)-u_{*}(t)\right|_{U^{\prime}}^{2} d t .
\end{aligned}
$$

Since by Eq. $58 \bar{u}_{n} \rightarrow u_{*}$ in $\mathbb{D}\left([0, T] ; U^{\prime}\right)$ and by Eq. $59 \sup _{t \in[0, T]}\left|\bar{u}_{n}(t)\right|_{H}^{2}<\infty, \overline{\mathbb{P}}$-a.s. and the embedding $H \hookrightarrow U^{\prime}$ is continuous, by the Dominated Convergence Theorem we infer that $\overline{\mathbb{P}}$-a.s., $\bar{u}_{n} \rightarrow u_{*}$ in $L^{2}\left(0, T ; U^{\prime}\right)$. Then

$$
\lim _{n \rightarrow \infty} \int_{0}^{T}\left|\left(\bar{u}_{n}(t)-u_{*}(t) \mid v\right)_{H}\right|^{2} d t=0 .
$$

Moreover, by the Hólder inequality and Eq. 59 for every $n \in \mathbb{N}$ and every $r \in$ $\left(1,2+\frac{\gamma}{2}\right]$

$$
\begin{aligned}
& \overline{\mathbb{E}}\left[\left|\int_{0}^{T}\right| \bar{u}_{n}(t)-\left.\left.u_{*}(t)\right|_{H} ^{2} d t\right|^{r}\right] \leq c \overline{\mathbb{E}}\left[\int_{0}^{T}\left(\left|\bar{u}_{n}(t)\right|_{H}^{2 r}+\left|u_{*}(t)\right|_{H}^{2 r}\right) d t\right] \\
& \leq \tilde{c} \overline{\mathbb{E}}\left[\sup _{t \in[0, T]}\left|\bar{u}_{n}(t)\right|_{H}^{2 r}\right] \leq \tilde{c} C_{1}(2 r)
\end{aligned}
$$


for some constants $c, \tilde{c}>0$. By Eqs. 66,65 and the Vitali Theorem we infer that

$$
\lim _{n \rightarrow \infty} \overline{\mathbb{E}}\left[\int_{0}^{T}\left|\left(\bar{u}_{n}(t)-u_{*}(t) \mid v\right)_{H}\right|^{2} d t\right]=0,
$$

i.e. Eq. 63 holds.

Let us move to the proof of Eq. 64. Note that by the Fubini Theorem, we have

$$
\begin{aligned}
& \left\|\mathscr{K}_{n}\left(\bar{u}_{n}, \bar{\eta}_{n}, \bar{W}_{n}, v\right)-\mathscr{K}\left(u_{*}, \eta_{*}, W_{*}, v\right)\right\|_{L^{2}([0, T] \times \bar{\Omega})}^{2} \\
& =\int_{0}^{T} \int_{\bar{\Omega}}\left|\mathscr{K}_{n}\left(\bar{u}_{n}, \bar{\eta}_{n}, \bar{W}_{n}, v\right)(t)-\mathscr{K}\left(u_{*}, \eta_{*}, W_{*}, v\right)(t)\right|^{2} d \overline{\mathbb{P}}(\omega) d t \\
& =\int_{0}^{T} \overline{\mathbb{E}}\left[\left|\mathscr{K}_{n}\left(\bar{u}_{n}, \bar{\eta}_{n}, \bar{W}_{n}, v\right)(t)-\mathscr{K}\left(u_{*}, \eta_{*}, W_{*}, v\right)(t)\right|^{2}\right] d t .
\end{aligned}
$$

We will prove that each term on the right hand side of Eq. 61 tends in $L^{2}([0, T] \times \bar{\Omega})$ to the corresponding term in Eq. 62.

Since by Eq. $58 \bar{u}_{n} \rightarrow u_{*}$ in $\mathbb{D}\left(0, T ; H_{w}\right) \overline{\mathbb{P}}$-a.s. and $u_{*}$ is continuous at $t=0$, we infer that $\left(\bar{u}_{n}(0) \mid v\right)_{H} \rightarrow\left(u_{*}(0) \mid v\right)_{H} \overline{\mathbb{P}}$-a.s. By Eq. 59 and the Vitali Theorem, we have

$$
\lim _{n \rightarrow \infty} \overline{\mathbb{E}}\left[\left|\left(\bar{u}_{n}(0)-u_{*}(0) \mid v\right)_{H}\right|^{2}\right]=0 \text {. }
$$

Hence

$$
\lim _{n \rightarrow \infty}\left\|\left(\bar{u}_{n}(0)-u_{*}(0) \mid v\right)_{H}\right\|_{L^{2}([0, T] \times \bar{\Omega})}^{2}=0 .
$$

By Eq. $58 \bar{u}_{n} \rightarrow u_{*}$ in $L_{w}^{2}(0, T ; V), \overline{\mathbb{P}}$-a.s. Moreover, since $v \in U, P_{n} v \rightarrow v$ in $V$, see Section 2.3. Thus by relation (15) we infer that

$$
\begin{aligned}
& \lim _{n \rightarrow \infty} \int_{0}^{t}\left\langle P_{n} \mathscr{A} \bar{u}_{n}(s) \mid v\right\rangle d s=\lim _{n \rightarrow \infty} \int_{0}^{t}\left(\left(\bar{u}_{n}(s) \mid P_{n} v\right)\right) d s \\
& =\int_{0}^{t}\left(\left(u_{*}(s) \mid v\right)\right) d s=\int_{0}^{t}\left\langle u_{*}(s) \mid v\right\rangle d s .
\end{aligned}
$$

By Eq. 15, Lemma 1, the Hólder inequality and Eq. 59 for all $t \in[0, T], r \in(0,2+\gamma]$ and $n \in \mathbb{N}$

$$
\begin{aligned}
& \overline{\mathbb{E}}\left[\left|\int_{0}^{t}\left\langle P_{n} \mathscr{A} \bar{u}_{n}(s) \mid v\right\rangle d s\right|^{2+r}\right]=\overline{\mathbb{E}}\left[\left|\int_{0}^{t}\left(\bar{u}_{n}(s) \mid(A-I) P_{n} v\right)_{H} d s\right|^{2+r}\right] \\
& \leq c\|v\|_{U}^{2+r} \overline{\mathbb{E}}\left[\int_{0}^{T}\left|\bar{u}_{n}(s)\right|_{H}^{2+r} d s\right] \leq \tilde{c} \overline{\mathbb{E}}\left[\sup _{0 \leq s \leq T}\left|\bar{u}_{n}(s)\right|_{H}^{2+r}\right] \leq \tilde{c} C_{1}(2+r)
\end{aligned}
$$

for some constants $c, \tilde{c}>0$. Therefore by Eqs. 68, 69 and the Vitali Theorem we infer that for all $t \in[0, T]$

$$
\lim _{n \rightarrow \infty} \overline{\mathbb{E}}\left[\left|\int_{0}^{t}\left\langle P_{n} \mathscr{A} \bar{u}_{n}(s)-\mathscr{A} u_{*}(s) \mid v\right\rangle d s\right|^{2}\right]=0 .
$$

Hence by Eq. 59 and the Dominated Convergence Theorem

$$
\lim _{n \rightarrow \infty} \int_{0}^{T} \overline{\mathbb{E}}\left[\left|\int_{0}^{t}\left\langle P_{n} \mathscr{A} \bar{u}_{n}(s)-\mathscr{A} u_{*}(s) \mid v\right\rangle d s\right|^{2}\right] d t=0 .
$$


Let us move to the nonlinear term. We will use the following auxiliary result proven in [9]. (We recall the proof in Appendix D.)

Lemma 6 (Lemma B.1 in [9]) Let $u \in L^{2}(0, T ; H)$ and let $\left(u_{n}\right)_{n}$ be a bounded sequence in $L^{2}(0, T ; H)$ such that $u_{n} \rightarrow u$ in $L^{2}\left(0, T ; H_{\mathrm{loc}}\right)$. Let $m>\frac{d}{2}+1$. Then for all $t \in[0, T]$ and all $\psi \in V_{m}$ :

$$
\lim _{n \rightarrow \infty} \int_{0}^{t}\left\langle B\left(u_{n}(s)\right) \mid \psi\right\rangle d s=\int_{0}^{t}\langle B(u(s)) \mid \psi\rangle d s .
$$

(Here $\langle\cdot \mid \cdot\rangle$ denotes the dual pairing between the space $V_{m}$ and $\left.V_{m}^{\prime}.\right)$

Let us fix $m>\frac{d}{2}+1$. Since by Eqs. 60 and 8 the sequence $\left(\bar{u}_{n}\right)$ is bounded in $L^{2}(0, T ; H)$ and by Eq. $58 \bar{u}_{n} \rightarrow u_{*}$ in $L^{2}\left(0, T ; H_{\text {loc }}\right) \overline{\mathbb{P}}$-a.s., by Lemma 6 we infer that $\overline{\mathbb{P}}$-a.s. for all $t \in[0, T]$ and all $v \in V_{m}$

$$
\lim _{n \rightarrow \infty} \int_{0}^{t}\left\langle B\left(\bar{u}_{n}(s)\right)-B\left(u_{*}(s)\right) \mid v\right\rangle d s=0 .
$$

It is easy to see that for sufficiently large $n \in \mathbb{N}$

$$
B_{n}\left(\bar{u}_{n}(s)\right)=P_{n} B\left(\bar{u}_{n}(s)\right), \quad s \in[0, T] .
$$

Moreover, if $v \in U$ then $P_{n} v \rightarrow v$ in $V_{m}$, see Section 2.3. Since $U \subset V_{m}$, we infer that for all $v \in U$ and all $t \in[0, T]$

$$
\lim _{n \rightarrow \infty} \int_{0}^{t}\left\langle B_{n}\left(\bar{u}_{n}(s)\right)-B\left(u_{*}(s)\right) \mid v\right\rangle d s=0 \quad \overline{\mathbb{P}} \text { a.s. }
$$

By the Hólder inequality, Eqs. 12 and 59 we obtain for all $t \in[0, T], r \in\left(0, \frac{\gamma}{2}\right]$ and $n \in \mathbb{N}$

$$
\begin{aligned}
& \overline{\mathbb{E}}\left[\left|\int_{0}^{t}\left\langle B_{n}\left(\bar{u}_{n}(s)\right) \mid v\right\rangle d s\right|^{2+r}\right] \leq \overline{\mathbb{E}}\left[t^{1+r}\|v\|_{V_{m}}^{2+r} \int_{0}^{t}\left|B_{n}\left(\bar{u}_{n}(s)\right)\right|_{V_{m}^{\prime}}^{2+r} d s\right] \\
& \leq C \overline{\mathbb{E}}\left[\int_{0}^{t}\left|\bar{u}_{n}(s)\right|_{H}^{2(2+r)} d s\right] \leq \tilde{C} \overline{\mathbb{E}}\left[\sup _{s \in[0, T]}\left|\bar{u}_{n}(s)\right|_{H}^{2(2+r)}\right] \leq \tilde{C} C_{1}(4+2 r) .
\end{aligned}
$$

In view of Eqs. 71 and 72, by the Vitali Theorem we obtain for all $t \in[0, T]$

$$
\lim _{n \rightarrow \infty} \overline{\mathbb{E}}\left[\left|\int_{0}^{t}\left\langle B_{n}\left(\bar{u}_{n}(s)\right)-B\left(u_{*}(s)\right) \mid v\right\rangle d s\right|^{2}\right]=0 .
$$

Since by Eq. 59 for all $t \in[0, T]$ and all $n \in \mathbb{N}$

$$
\left.\overline{\mathbb{E}}\left[\left|\int_{0}^{t}\left\langle B_{n}\left(\bar{u}_{n}(s)\right) \mid v\right\rangle d s\right|^{2}\right] \leq\left. c \overline{\mathbb{E}}\left[\sup _{s \in[0, T]} \mid \bar{u}_{n}(s)\right)\right|_{H} ^{4}\right] \leq c C_{1}(4)
$$


for some $c>0$, by Eq. 73 and the Dominated Convergence Theorem, we infer that

$$
\lim _{n \rightarrow \infty} \int_{0}^{T} \overline{\mathbb{E}}\left[\left|\int_{0}^{t}\left\langle B_{n}\left(\bar{u}_{n}(s)\right)-B\left(u_{*}(s)\right) \mid v\right\rangle d s\right|^{2}\right] d t=0
$$

Let us move to the noise terms. Let us assume first that $v \in \mathscr{V}$. For all $t \in[0, T]$ we have

$$
\begin{aligned}
& \int_{0}^{t} \int_{Y}\left|\left\langle F\left(s, \bar{u}_{n}\left(s^{-}\right) ; y\right)-F\left(s, u_{*}\left(s^{-}\right) ; y\right) \mid v\right\rangle\right|^{2} d v(y) d s \\
& \int_{0}^{t} \int_{Y}\left|\left(F\left(s, \bar{u}_{n}\left(s^{-}\right) ; y\right)-\left.F\left(s, u_{*}\left(s^{-}\right) ; y\right)|v\rangle_{H}\right|^{2} d v(y) d s\right.\right. \\
& =\int_{0}^{t} \int_{Y}\left|\tilde{F}_{v}\left(\bar{u}_{n}\right)(s, y)-\tilde{F}_{v}\left(u_{*}\right)(s, y)\right|^{2} d v(y) d s \\
& \leq \int_{0}^{T} \int_{Y}\left|\tilde{F}_{v}\left(\bar{u}_{n}\right)(s, y)-\tilde{F}_{v}\left(u_{*}\right)(s, y)\right|^{2} d v(y) d s \\
& =\left\|\tilde{F}_{v}\left(\bar{u}_{n}\right)-\tilde{F}_{v}\left(u_{*}\right)\right\|_{L^{2}([0, T] \times Y ; \mathbb{R})}^{2},
\end{aligned}
$$

where $\tilde{F}_{v}$ is the mapping defined by Eq. 33 . Since by Eq. $58 \bar{u}_{n} \rightarrow u_{*}$ in $L^{2}\left(0, T ; H_{\mathrm{loc}}\right)$, $\overline{\mathbb{P}}$-a.s., by assumption (F.3) we infer that for all $t \in[0, T]$

$$
\lim _{n \rightarrow \infty} \int_{0}^{t} \int_{Y}\left|\left(F\left(s, \bar{u}_{n}\left(s^{-}\right) ; y\right)-F\left(s, u_{*}\left(s^{-}\right) ; y\right) \mid v\right)_{H}\right|^{2} d v(y) d s=0 .
$$

Moreover, by inequality (32) in assumption (F.2) and by Eq. 59 for every $t \in[0, T]$ every $r \in\left(1,2+\frac{\gamma}{2}\right]$ and every $n \in \mathbb{N}$ the following inequalities hold

$$
\begin{aligned}
& \overline{\mathbb{E}}\left[\left.\left.\left|\int_{0}^{t} \int_{Y}\right|\left(F\left(s, \bar{u}_{n}\left(s^{-}\right) ; y\right)-F\left(s, u_{*}\left(s^{-}\right) ; y\right) \mid v\right)_{H}\right|^{2} d v(y) d s\right|^{r}\right] \\
& \leq 2^{r}|v|_{H}^{2 r} \overline{\mathbb{E}}\left[\left|\int_{0}^{t} \int_{Y}\left\{\left|F\left(s, \bar{u}_{n}\left(s^{-}\right) ; y\right)\right|_{H}^{2}+\left|F\left(s, u_{*}\left(s^{-}\right) ; y\right)\right|_{H}^{2}\right\} d v(y) d s\right|^{r}\right] \\
& \leq 2^{r} C_{2}^{r}|v|_{H}^{2 r} \overline{\mathbb{E}}\left[\left|\int_{0}^{t}\left\{2+\left|\bar{u}_{n}(s)\right|_{H}^{2}+\left|u_{*}(s)\right|_{H}^{2}\right\} d s\right|^{r}\right] \leq c\left(1+\overline{\mathbb{E}}\left[\sup _{s \in[0, T]}\left|\bar{u}_{n}(s)\right|_{H}^{2 r}\right]\right) \\
& \leq c\left(1+C_{1}(2 r)\right)
\end{aligned}
$$

for some constant $c>0$. Thus by Eqs. 75, 76 and the Vitali Theorem for all $t \in[0, T]$

$$
\lim _{n \rightarrow \infty} \overline{\mathbb{E}}\left[\int_{0}^{t} \int_{Y}\left|\left\langle F\left(s, \bar{u}_{n}\left(s^{-}\right) ; y\right)-F\left(s, u_{*}\left(s^{-}\right) ; y\right) \mid v\right\rangle\right|^{2} d \nu(y) d s\right]=0, \quad v \in \mathscr{V} .
$$


Let now $v \in H$ and let $\varepsilon>0$. Since $\mathscr{V}$ is dense in $H$, there exists $v_{\varepsilon} \in \mathscr{V}$ such that $\left|v-v_{\varepsilon}\right|_{H}^{2}<\varepsilon$. By Eq. 32 the following inequalities hold

$$
\begin{aligned}
& \int_{0}^{t} \int_{Y}\left|\left\langle F\left(s, \bar{u}_{n}\left(s^{-}\right) ; y\right)-F\left(s, u_{*}\left(s^{-}\right) ; y\right) \mid v\right\rangle\right|^{2} d v(y) d s \\
& \leq 2 \int_{0}^{t} \int_{Y}\left|\left\langle F\left(s, \bar{u}_{n}\left(s^{-}\right) ; y\right)-F\left(s, u_{*}\left(s^{-}\right) ; y\right) \mid v-v_{\varepsilon}\right\rangle\right|^{2} d v(y) d s \\
& \quad+2 \int_{0}^{t} \int_{Y}\left|\left\langle F\left(s, \bar{u}_{n}\left(s^{-}\right) ; y\right)-F\left(s, u_{*}\left(s^{-}\right) ; y\right) \mid v_{\varepsilon}\right\rangle\right|^{2} d v(y) d s \\
& \leq 4 C_{2} \varepsilon^{2} \int_{0}^{t}\left\{2+\left|\bar{u}_{n}(s)\right|_{H}^{2}+\left|u_{*}(s)\right|_{H}^{2}\right\} d s \\
& \quad+2 \int_{0}^{t} \int_{Y}\left|\left\langle F\left(s, \bar{u}_{n}\left(s^{-}\right) ; y\right)-F\left(s, u_{*}\left(s^{-}\right) ; y\right) \mid v_{\varepsilon}\right\rangle\right|^{2} d v(y) d s .
\end{aligned}
$$

Hence by Eq. 59

$$
\begin{aligned}
& \overline{\mathbb{E}}\left[\int_{0}^{t} \int_{Y}\left|\left\langle F\left(s, \bar{u}_{n}\left(s^{-}\right) ; y\right)-F\left(s, u_{*}\left(s^{-}\right) ; y\right) \mid v\right\rangle\right|^{2} d v(y) d s\right] \\
& \leq 4 C_{2} \varepsilon^{2} \overline{\mathbb{E}}\left[\int_{0}^{t}\left\{2+\left|\bar{u}_{n}(s)\right|_{H}^{2}+\left|u_{*}(s)\right|_{H}^{2}\right\} d s\right] \\
& \quad+2 \overline{\mathbb{E}}\left[\int_{0}^{t} \int_{Y}\left|\left\langle F\left(s, \bar{u}_{n}\left(s^{-}\right) ; y\right)-F\left(s, u_{*}\left(s^{-}\right) ; y\right) \mid v_{\varepsilon}\right\rangle\right|^{2} d v(y) d s\right] \\
& \leq \tilde{c} \varepsilon^{2}+2 \overline{\mathbb{E}}\left[\int_{0}^{t} \int_{Y}\left|\left\langle F\left(s, \bar{u}_{n}\left(s^{-}\right) ; y\right)-F\left(s, u_{*}\left(s^{-}\right) ; y\right) \mid v_{\varepsilon}\right\rangle\right|^{2} d v(y) d s\right] .
\end{aligned}
$$

Passing to the upper limit as $n \rightarrow \infty$ in the above inequality, by Eq. 77 we obtain

$$
\limsup _{n \rightarrow \infty} \overline{\mathbb{E}}\left[\int_{0}^{t} \int_{Y}\left|\left\langle F\left(s, \bar{u}_{n}\left(s^{-}\right) ; y\right)-F\left(s, u_{*}\left(s^{-}\right) ; y\right) \mid v\right\rangle\right|^{2} d \nu(y) d s\right] \leq \tilde{c} \varepsilon^{2} .
$$

Since $\varepsilon>0$ was chosen in an arbitrary way, we infer that for all $v \in H$

$$
\lim _{n \rightarrow \infty} \overline{\mathbb{E}}\left[\int_{0}^{t} \int_{Y}\left|\left\langle F\left(s, \bar{u}_{n}\left(s^{-}\right) ; y\right)-F\left(s, u_{*}\left(s^{-}\right) ; y\right) \mid v\right\rangle\right|^{2} d v(y) d s\right]=0 .
$$

Moreover, since the restriction of $P_{n}$ to the space $H$ is the $(\cdot \mid \cdot)_{H}$-projection onto $H_{n}$, see Section 2.3, we infer that also

$$
\lim _{n \rightarrow \infty} \overline{\mathbb{E}}\left[\int_{0}^{t} \int_{Y}\left|\left\langle P_{n} F\left(s, \bar{u}_{n}\left(s^{-}\right) ; y\right)-F\left(s, u_{*}\left(s^{-}\right) ; y\right) \mid v\right\rangle\right|^{2} d v(y) d s\right]=0, \quad v \in H .
$$

Hence by the properties of the integral with respect to the compensated Poisson random measure and the fact that $\bar{\eta}_{n}=\eta_{*}$, we have

$$
\lim _{n \rightarrow \infty} \overline{\mathbb{E}}\left[\mid \int_{0}^{t} \int_{Y}\left\langle P_{n} F\left(s, \bar{u}_{n}\left(s^{-}\right) ; y\right)-\left.F\left(s, u_{*}\left(s^{-}\right) ; y\right)|v| \tilde{\eta}_{*}(d s, d y)\right|^{2}\right]=0 .\right.
$$


Moreover, by inequality (32) in assumption (F.2) and by Eq. 59 we obtain the following inequalities

$$
\begin{aligned}
& \overline{\mathbb{E}}\left[\mid \int_{0}^{t} \int_{Y}\left\langle P_{n} F\left(s, \bar{u}_{n}\left(s^{-}\right) ; y\right)-\left.F\left(s, u_{*}\left(s^{-}\right) ; y\right)|v| \tilde{\eta}_{*}(d s, d y)\right|^{2}\right]\right. \\
& =\overline{\mathbb{E}}\left[\int_{0}^{t} \int_{Y}\left|\left(P_{n} F\left(s, \bar{u}_{n}\left(s^{-}\right) ; y\right)-F\left(s, u_{*}\left(s^{-}\right) ; y\right) \mid v\right)_{H}\right|^{2} v(d y) d s\right] \\
& \leq 2|v|_{H}^{2} \overline{\mathbb{E}}\left[\int_{0}^{t} \int_{Y}\left\{\left|P_{n} F\left(s, \bar{u}_{n}\left(s^{-}\right) ; y\right)\right|_{H}^{2}+\left|F\left(s, u_{*}\left(s^{-}\right) ; y\right)\right|_{H}^{2}\right\} v(d y) d s\right] \\
& \leq 2 C_{2}|v|_{H}^{2} \overline{\mathbb{E}}\left[\int_{0}^{t}\left\{2+\left|\bar{u}_{n}(s)\right|_{H}^{2}+\left|u_{*}(s)\right|_{H}^{2}\right\} d s\right] \leq c\left(1+\overline{\mathbb{E}}\left[\sup _{s \in[0, T]}\left|\bar{u}_{n}(s)\right|_{H}^{2}\right]\right) \\
& \leq c\left(1+C_{1}(2)\right) .
\end{aligned}
$$

By Eqs. 78, 79 and the Dominated Convergence Theorem, we have for all $v \in H$

$$
\lim _{n \rightarrow \infty} \int_{0}^{T} \overline{\mathbb{E}}\left[\left|\int_{0}^{t} \int_{Y}\left\langle P_{n} F\left(s, \bar{u}_{n}\left(s^{-}\right) ; y\right)-F\left(s, u_{*}\left(s^{-}\right) ; y\right) \mid v\right\rangle \tilde{\eta}_{*}(d s, d y)\right|^{2}\right] d t=0 .
$$

Since $U \subset H$, Eq. 80 holds for all $v \in U$, as well.

Let us move to the second part of the noise. Let us assume first that $v \in \mathscr{V}$. We have

$$
\begin{aligned}
& \int_{0}^{t}\left\|\left\langle G\left(s, \bar{u}_{n}(s)\right)-G\left(s, u_{*}(s)\right) \mid v\right\rangle\right\|_{\mathscr{L}_{\mathrm{HS}}\left(Y_{w} ; \mathbb{R}\right)}^{2} d s \\
& =\int_{0}^{t}\left\|\left(G\left(s, \bar{u}_{n}(s)\right)-G\left(s, u_{*}(s)\right) \mid v\right)_{H}\right\|_{\mathscr{L}_{\mathrm{HS}}\left(Y_{w} ; \mathbb{R}\right)}^{2} d s \\
& =\int_{0}^{t}\left\|\tilde{G}_{v}\left(\bar{u}_{n}\right)(s)-\tilde{G}_{v}\left(u_{*}\right)(s)\right\|_{\mathscr{L}_{\mathrm{HS}}\left(Y_{w} ; \mathbb{R}\right)}^{2} d s \\
& \leq \int_{0}^{T}\left\|\tilde{G}_{v}\left(\bar{u}_{n}\right)(s)-\tilde{G}_{v}\left(u_{*}\right)(s)\right\|_{\mathscr{L}_{\mathrm{HS}}\left(Y_{w} ; \mathbb{R}\right)}^{2} d s \\
& =\left\|\tilde{G}_{v}\left(\bar{u}_{n}\right)-\tilde{G}_{v}\left(u_{*}\right)\right\|_{L^{2}\left([0, T] ; \mathscr{L}_{\mathrm{HS}}\left(Y_{w} ; \mathbb{R}\right)\right)}^{2},
\end{aligned}
$$

where $\tilde{G}_{v}$ is the mapping defined by Eq. 37 . Since by Eq. $58 \bar{u}_{n} \rightarrow u_{*}$ in $L^{2}\left(0, T ; H_{\mathrm{loc}}\right.$, $\overline{\mathbb{P}}$-a.s., by the second part of assumption (G.3) we infer that for all $t \in[0, T]$ and all $v \in \mathscr{V}$

$$
\lim _{n \rightarrow \infty} \int_{0}^{t}\left\|\left\langle G\left(s, \bar{u}_{n}(s)\right)-G\left(s, u_{*}(s)\right) \mid v\right\rangle\right\|_{\mathscr{L}_{\mathrm{HS}}\left(Y_{w} ; \mathbb{R}\right)}^{2} d s=0 .
$$


Moreover, by Eqs. 36 and 59 we see that for every $t \in[0, T]$ every $r \in\left(1,2+\frac{\gamma}{2}\right]$ and every $n \in \mathbb{N}$

$$
\begin{aligned}
& \overline{\mathbb{E}}\left[\left|\int_{0}^{t}\left\|\left\langle G\left(s, \bar{u}_{n}(s)\right)-G\left(s, u_{*}(s)\right) \mid v\right\rangle\right\|_{\mathscr{L}_{\mathrm{HS}}\left(Y_{w} ; \mathbb{R}\right)}^{2} d s\right|^{r}\right] \\
& \leq c \overline{\mathbb{E}}\left[\|v\|_{V}^{2 r} \cdot \int_{0}^{t}\left\{\left\|G\left(s, \bar{u}_{n}(s)\right)\right\|_{\mathscr{L}_{\mathrm{HS}}\left(Y_{W} ; V^{\prime}\right)}^{2 r}+\left\|G\left(s, u_{*}(s)\right)\right\|_{\mathscr{L}_{\mathrm{HS}}\left(Y_{W} ; V^{\prime}\right)}^{2 r}\right\} d s\right] \\
& \leq c_{1} \overline{\mathbb{E}}\left[\int_{0}^{T}\left(1+\left|\bar{u}_{n}(s)\right|_{H}^{2 r}+\left|u_{*}(s)\right|_{H}^{2 r}\right) d s\right] \\
& \left.\leq \tilde{c}\left\{1+\overline{\mathbb{E}}\left[\sup _{s \in[0, T]}\left|\bar{u}_{n}(s)\right|_{H}^{2 r}+\sup _{s \in[0, T]}\left|u_{*}(s)\right|_{H}^{2 r}\right)\right]\right\} \leq \tilde{c}\left(1+2 C_{1}(2 r)\right)
\end{aligned}
$$

for some positive constants $c, c_{1}, \tilde{c}$. Thus by Eqs. 81, 82 and the Vitali Theorem

$$
\lim _{n \rightarrow \infty} \overline{\mathbb{E}}\left[\int_{0}^{t}\left\|\left\langle G\left(s, \bar{u}_{n}(s)\right)-G\left(s, u_{*}(s)\right) \mid v\right\rangle\right\|_{\mathscr{L}_{\mathrm{HS}}\left(Y_{w} ; \mathbb{R}\right)}^{2} d s\right]=0 \quad \text { for all } v \in \mathscr{V} .
$$

Let now $v \in V$ and let $\varepsilon>0$. Since $\mathscr{V}$ is dense in $V$, there exists $v_{\varepsilon} \in \mathscr{V}$ such that $\left\|v-v_{\varepsilon}\right\|_{V} \leq \varepsilon$. We have the following inequalities

$$
\begin{aligned}
& \int_{0}^{t}\left\|\left\langle G\left(s, \bar{u}_{n}(s)\right)-G\left(s, u_{*}(s)\right) \mid v\right\rangle\right\|_{\mathscr{L}_{\mathrm{HS}}\left(Y_{w} ; \mathbb{R}\right)}^{2} d s \\
& \leq 2 \int_{0}^{t}\left\|\left\langle G\left(s, \bar{u}_{n}(s)\right)-G\left(s, u_{*}(s)\right) \mid v-v_{\varepsilon}\right\rangle\right\|_{\mathscr{L}_{\mathrm{HS}}\left(Y_{w} ; \mathbb{R}\right)}^{2} d s \\
& \quad+2 \int_{0}^{t}\left\|\left\langle G\left(s, \bar{u}_{n}(s)\right)-G\left(s, u_{*}(s)\right) \mid v_{\varepsilon}\right\rangle\right\|_{\mathscr{L}_{\mathrm{HS}}\left(Y_{w} ; \mathbb{R}\right)}^{2} d s .
\end{aligned}
$$

Moreover, by inequality (36) in assumption (G.3), we obtain the following estimates

$$
\begin{aligned}
& \int_{0}^{t}\left\|\left\langle G\left(s, \bar{u}_{n}(s)\right)-G\left(s, u_{*}(s)\right) \mid v-v_{\varepsilon}\right\rangle\right\|_{\mathscr{L}+\left(Y_{w} ; \mathbb{R}\right)}^{2} d s \\
& \leq\left\|v-v_{\varepsilon}\right\|_{V}^{2} \int_{0}^{t}\left\|G\left(s, \bar{u}_{n}(s)\right)-G\left(s, u_{*}(s)\right)\right\|_{\mathscr{L}_{\mathrm{HS}}\left(Y_{w} ; V^{\prime}\right)}^{2} d s \\
& \leq c\left(1+\sup _{s \in[0, T]}\left|\bar{u}_{n}(s)\right|_{H}^{2}+\sup _{s \in[0, T]}\left|u_{*}(s)\right|_{H}^{2}\right) \varepsilon^{2}
\end{aligned}
$$

for some $c>0$. Thus by Eq. 59 we obtain the following inequalities

$$
\begin{aligned}
& \overline{\mathbb{E}}\left[\int_{0}^{t}\left\|\left\langle G\left(s, \bar{u}_{n}(s)\right)-G\left(s, u_{*}(s)\right) \mid v\right\rangle\right\|_{\mathscr{L}_{\mathrm{HS}}\left(Y_{w} ; \mathbb{R}\right)}^{2} d s\right] \\
& \leq 2 c\left\{1+2 C_{1}(2)\right\} \varepsilon^{2}+2 \overline{\mathbb{E}}\left[\int_{0}^{t}\left\|\left\langle G\left(s, \bar{u}_{n}(s)\right)-G\left(s, u_{*}(s)\right) \mid v_{\varepsilon}\right\rangle\right\|_{\mathscr{L}_{\mathrm{HS}}\left(Y_{w} ; \mathbb{R}\right)}^{2} d s\right] .
\end{aligned}
$$

Passing to the upper limit as $n \rightarrow \infty$ by Eq. 83 we infer that for all $v \in V$

$$
\limsup _{n \rightarrow \infty} \overline{\mathbb{E}}\left[\int_{0}^{t}\left\|\left\langle G\left(s, \bar{u}_{n}(s)\right)-G\left(s, u_{*}(s)\right) \mid v\right\rangle\right\|_{\mathscr{L}_{\mathrm{HS}}\left(Y_{w} ; \mathbb{R}\right)}^{2} d s\right] \leq C \varepsilon^{2},
$$


where $C=2 c\left\{1+2 C_{1}(2)\right\}$. Since $\varepsilon>0$ was chosen in an arbitrary way, we infer that

$$
\lim _{n \rightarrow \infty} \overline{\mathbb{E}}\left[\int_{0}^{t}\left\|\left\langle G\left(s, \bar{u}_{n}(s)\right)-G\left(s, u_{*}(s)\right) \mid v\right\rangle\right\|_{\mathscr{L}_{\mathrm{HS}}\left(Y_{w} ; \mathbb{R}\right)}^{2} d s\right]=0 \quad \text { for all } v \in V .
$$

For every $v \in V$ and every $s \in[0, T]$ we have

$$
\begin{aligned}
& \left\langle P_{n} G\left(s, \bar{u}_{n}(s)\right)-G\left(s, u_{*}(s)\right) \mid v\right\rangle=\left\langle G\left(s, \bar{u}_{n}(s)\right) \mid P_{n} v\right\rangle-\left\langle G\left(s, u_{*}(s)\right) \mid v\right\rangle \\
& =\left\langle G\left(s, \bar{u}_{n}(s)\right) \mid P_{n} v-v\right\rangle+\left\langle G\left(s, \bar{u}_{n}(s)\right)-G\left(s, u_{*}(s)\right) \mid v\right\rangle \\
& \leq\left\|G\left(s, \bar{u}_{n}(s)\right)\right\| \mathscr{L}_{\mathrm{HS}\left(Y_{W}, V^{\prime}\right)}\left\|P_{n} v-v\right\|_{V}+\left\langle G\left(s, \bar{u}_{n}(s)\right)-G\left(s, u_{*}(s)\right) \mid v\right\rangle .
\end{aligned}
$$

Thus by inequality (36) in assumption (G.3) and by Eq. 59 we obtain

$$
\begin{aligned}
\overline{\mathbb{E}} & {\left[\int_{0}^{t}\left\|\left\langle P_{n} G\left(s, \bar{u}_{n}(s)\right)-G\left(s, u_{*}(s)\right) \mid v\right\rangle\right\|_{\mathscr{L}_{\mathrm{HS}}\left(Y_{w} ; \mathbb{R}\right)}^{2} d s\right] } \\
\leq & 2\left\|P_{n} v-v\right\|_{V}^{2} \overline{\mathbb{E}}\left[\int_{0}^{T}\left\|G\left(s, \bar{u}_{n}(s)\right)\right\|_{\mathscr{L}_{\mathrm{HS}}\left(Y_{W}, V^{\prime}\right)}^{2} d s\right] \\
& +2 \overline{\mathbb{E}}\left[\int_{0}^{t}\left\|\left\langle G\left(s, \bar{u}_{n}(s)\right)-G\left(s, u_{*}(s)\right) \mid v\right\rangle\right\|_{\mathscr{L}_{\mathrm{HS}}\left(Y_{w} ; \mathbb{R}\right)}^{2} d s\right] \\
\leq & 2 C\left\|P_{n} v-v\right\|_{V}^{2} \overline{\mathbb{E}}\left[\int_{0}^{T}\left(1+\left|\bar{u}_{n}(s)\right|_{H}^{2}\right) d s\right] \\
& +2 \overline{\mathbb{E}}\left[\int_{0}^{t}\left\|\left\langle G\left(s, \bar{u}_{n}(s)\right)-G\left(s, u_{*}(s)\right) \mid v\right\rangle\right\|_{\mathscr{L}_{\mathrm{HS}}\left(Y_{w} ; \mathbb{R}\right)}^{2} d s\right] \\
\leq & 2 C T\left(1+C_{1}(2)\right)\left\|P_{n} v-v\right\|_{V}^{2} \\
& +2 \overline{\mathbb{E}}\left[\int_{0}^{t}\left\|\left\langle G\left(s, \bar{u}_{n}(s)\right)-G\left(s, u_{*}(s)\right) \mid v\right\rangle\right\|_{\mathscr{L}_{\mathrm{HS}}\left(Y_{w} ; \mathbb{R}\right)}^{2} d s\right] .
\end{aligned}
$$

Since $U \subset V$ and $\left\|P_{n} v-v\right\|_{V} \rightarrow 0$ for all $v \in U$, see Section 2.3, by Eq. 84 we infer that

$$
\lim _{n \rightarrow \infty} \overline{\mathbb{E}}\left[\int_{0}^{t}\left\|\left\langle\mathbb{P} n G\left(s, \bar{u}_{n}(s)\right)-G\left(s, u_{*}(s)\right) \mid v\right\rangle\right\|_{\mathscr{L}_{\mathrm{HS}}\left(Y_{w} ; \mathbb{R}\right)}^{2} d s\right]=0 \quad \text { for all } v \in U
$$

Hence by the properties of the Itô integral we infer that for all $t \in[0, T]$ and all $v \in U$

$$
\lim _{n \rightarrow \infty} \overline{\mathbb{E}}\left[\left|\left\langle\int_{0}^{t}\left[P_{n} G\left(s, \bar{u}_{n}(s)\right)-G\left(s, u_{*}(s)\right)\right] d W_{*}(s) \mid v\right\rangle\right|^{2}\right]=0 .
$$


Moreover, by the Itô isometry, inequality (36) in assumption (G.3), and Eq. 59 we have for all $t \in[0, T]$ and all $n \in \mathbb{N}$

$$
\begin{aligned}
& \overline{\mathbb{E}}\left[\left|\left\langle\int_{0}^{t}\left[P_{n} G\left(s, \bar{u}_{n}(s)\right)-G\left(s, u_{*}(s)\right)\right] d W_{*}(s) \mid v\right\rangle\right|^{2}\right] \\
& =\overline{\mathbb{E}}\left[\int_{0}^{t}\left\|\left\langle P_{n} G\left(s, \bar{u}_{n}(s)\right)-G\left(s, u_{*}(s)\right) \mid v\right\rangle\right\|_{\mathscr{L}_{\mathrm{HS}}\left(Y_{w} ; \mathbb{R}\right)}^{2} d s\right] \\
& \left.\leq c\left\{1+\overline{\mathbb{E}}\left[\sup _{s \in[0, T]}\left|\bar{u}_{n}(s)\right|_{H}^{2}+\sup _{s \in[0, T]}\left|u_{*}(s)\right|_{H}^{2}\right)\right]\right\} \leq c\left(1+2 C_{1}(2)\right)
\end{aligned}
$$

for some $c>0$. By Eqs. 85, 86 and the Dominated Convergence Theorem we infer that

$$
\lim _{n \rightarrow \infty} \int_{0}^{T} \overline{\mathbb{E}}\left[\left|\left\langle\int_{0}^{t}\left[P_{n} G\left(s, \bar{u}_{n}(s)\right)-G\left(s, u_{*}(s)\right)\right] d W_{*}(s) \mid v\right\rangle\right|^{2}\right]=0 .
$$

By Eqs. 67, 70, 74, 80 and 87 the proof of Eq. 64 is complete.

Step $2^{0}$ Since $u_{n}$ is a solution of the Galerkin equation, for all $t \in[0, T]$

$$
\left(u_{n}(t) \mid v\right)_{H}=\mathscr{K}_{n}\left(u_{n}, \eta_{n}, W_{n}, v\right)(t), \quad \mathbb{P} \text {-a.s. }
$$

In particular,

$$
\int_{0}^{T} \mathbb{E}\left[\left|\left(u_{n}(t) \mid v\right) H-\mathscr{K}_{n}\left(u_{n}, \eta_{n}, W_{n}, v\right)(t)\right|^{2}\right] d t=0 .
$$

Since $\mathscr{L}\left(u_{n}, \eta_{n}, W_{n}\right)=\mathscr{L}\left(\bar{u}_{n}, \bar{\eta}_{n}, \bar{W}_{n}\right)$,

$$
\int_{0}^{T} \overline{\mathbb{E}}\left[\left|\left(\bar{u}_{n} t \mid v\right)_{H}-\mathscr{K}_{n}\left(\bar{u}_{n}, \bar{\eta}_{n}, \bar{W}_{n}, v\right)(t)\right|^{2}\right] d t=0 .
$$

Moreover, by Eqs. 63 and 64

$$
\int_{0}^{T} \overline{\mathbb{E}}\left[\left|\left(u_{*}(t) \mid v\right)_{H}-\mathscr{K}\left(u_{*}, \eta_{*}, W_{*}, v\right)(t)\right|^{2}\right] d t=0 .
$$

Hence for $l$-almost all $t \in[0, T]$ and $\overline{\mathbb{P}}$-almost all $\omega \in \bar{\Omega}$

$$
\left(u_{*}(t) \mid v\right)_{H}-\mathscr{K}\left(u_{*}, \eta_{*}, W_{*}, v\right)(t)=0,
$$

i.e. for $l$-almost all $t \in[0, T]$ and $\overline{\mathbb{P}}$-almost all $\omega \in \bar{\Omega}$

$$
\begin{aligned}
\left(u_{*}(t) \mid v\right)_{H}- & \left(u_{*}(0) \mid v\right)_{H}+\int_{0}^{t}\left\langle\mathscr{A} u_{*}(s) \mid v\right\rangle d s+\int_{0}^{t}\left\langle B\left(u_{*}(s), u_{*}(s)\right) \mid v\right\rangle d s \\
& -\int_{0}^{t}\langle f(s) \mid v\rangle d s-\int_{0}^{t} \int_{Y}\left(F\left(s, u_{*}(s) ; y\right) \mid v\right)_{H} \tilde{\eta}_{*}(d s, d y) \\
& -\left\langle\int_{0}^{t} G\left(s, u_{*}(s)\right) d W_{*}(s) \mid v\right\rangle=0 .
\end{aligned}
$$

Since $u_{*}$ is $\mathscr{Z}$-valued random variable, in particular $u_{*} \in \mathbb{D}\left([0, T] ; H_{w}\right)$, i.e. $u_{*}$ is weakly càdlàg. Hence the function on the left-hand side of the above equality is 
càdlàg with respect to $t$. Since two càdlàg functions equal for $l$-almost all $t \in[0, T]$ must be equal for all $t \in[0, T]$, we infer that for all $t \in[0, T]$ and all $v \in U$

$$
\begin{aligned}
\left(u_{*}(t) \mid v\right)_{H}- & \left(u_{*}(0) \mid v\right)_{H}+\int_{0}^{t}\left\langle\mathscr{A} u_{*}(s) \mid v\right\rangle d s+\int_{0}^{t}\left\langle B\left(u_{*}(s), u_{*}(s)\right) \mid v\right\rangle d s \\
& -\int_{0}^{t}\langle f(s) \mid v\rangle d s-\int_{0}^{t} \int_{Y}\left(F\left(s, u_{*}(s) ; y\right) \mid v\right)_{H} \tilde{\eta}_{*}(d s, d y) \\
& -\left\langle\int_{0}^{t} G\left(s, u_{*}(s)\right) d W_{*}(s) \mid v\right\rangle=0 \quad \overline{\mathbb{P}} \text {-a.s. }
\end{aligned}
$$

Since $U$ is dense in $\mathrm{V}$, we infer that the above equality holds for all $v \in V$. Putting $\bar{u}:=u_{*}, \bar{\eta}:=\eta_{*}$ and $\bar{W}:=W_{*}$, we infer that the system $(\bar{\Omega}, \overline{\mathscr{F}}, \overline{\mathbb{P}}, \overline{\mathbb{F}}, \bar{u}, \bar{\eta}, \bar{W})$ is a martingale solution of the Eq. (30). The proof of Theorem 3 is thus complete.

Acknowledgement The author would like to thank Zdzisław Brzeźniak for very helpful comments.

Open Access This article is distributed under the terms of the Creative Commons Attribution License which permits any use, distribution, and reproduction in any medium, provided the original author(s) and the source are credited.

\section{Appendix A}

\section{A.1 The Aldous Condition}

Here $(\mathbb{S}, \rho)$ is a separable and complete metric space. Let $(\Omega, \mathscr{F}, \mathbb{P})$ be a probability space with filtration $\mathbb{F}:=\left(\mathscr{F}_{t}\right)_{t \in[0, T]}$ satisfying the usual hypotheses, see [21], and let $\left(X_{n}\right)_{n \in \mathbb{N}}$ be a sequence of càdlàg, $\mathbb{F}$-adapted and $\mathbb{S}$-valued processes.

Definition 4 (See [19]) We say that the sequence $\left(X_{n}\right)$ of $\mathbb{S}$-valued random variables satifies condition $[\tilde{\mathbf{T}}]$ iff

$$
\begin{aligned}
\forall \varepsilon>0 \quad \forall \eta>0 \quad \exists \delta>0: & \\
& \sup _{n \in \mathbb{N}} \mathbb{P}\left\{w_{[0, T]}\left(X_{n}, \delta\right)>\eta\right\} \leq \varepsilon .
\end{aligned}
$$

Let us recall that $w_{[0, T]}$ stands for the modulus defined by Eq. 21.

Remark Let $\mathbb{P}_{n}$ denote the law of $X_{n}$ on $\mathbb{D}([0, T], \mathbb{S})$. For fixed $\eta>0$ and $\delta>0$ we denote

$$
C_{\eta, \delta}:=\left\{u \in \mathbb{D}([0, T], \mathbb{S}): w_{[0, T]}(u, \delta) \geq \eta\right\}
$$

Then condition

$$
\mathbb{P}\left\{w_{[0, T]}\left(X_{n}, \delta\right)>\eta\right\} \leq \varepsilon
$$

is equivalent to

$$
\mathbb{P}_{n}\left(C_{\eta, \delta}\right) \leq \varepsilon
$$


Lemma 7 Assume that $\left(X_{n}\right)$ satifies condition $[\tilde{\mathbf{T}}]$. Let $\mathbb{P}_{n}$ be the law of $X_{n}$ on $\mathbb{D}([0, T], \mathbb{S}), n \in \mathbb{N}$. Then for every $\varepsilon>0$ there exists a subset $A_{\varepsilon} \subset \mathbb{D}([0, T], \mathbb{S})$ such that

$$
\sup _{n \in \mathbb{N}} \mathbb{P}_{n}\left(A_{\varepsilon}\right) \geq 1-\varepsilon
$$

and

$$
\lim _{\delta \rightarrow 0} \sup _{u \in A_{\varepsilon}} w_{[0, T]}(u, \delta)=0 .
$$

Proof Fix $\varepsilon>0$. By $[\tilde{\mathbf{T}}]$, for each $k \in \mathbb{N}$ there exists $\delta_{k}>0$ such that

$$
\sup _{n \in \mathbb{N}} \mathbb{P}\left\{w_{[0, T]}\left(X_{n}, \delta_{k}\right)>\frac{1}{k}\right\} \leq \frac{\varepsilon}{2^{k+1}} .
$$

Then

$$
\sup _{n \in \mathbb{N}} \mathbb{P}\left\{w_{[0, T]}\left(X_{n}, \delta_{k}\right) \leq \frac{1}{k}\right\} \geq 1-\frac{\varepsilon}{2^{k+1}}
$$

or equivalently

$$
\sup _{n \in \mathbb{N}} \mathbb{P}_{n}\left\{u \in \mathbb{D}([0, T], \mathbb{S}): w_{[0, T]}\left(u, \delta_{k}\right) \leq \frac{1}{k}\right\} \geq 1-\frac{\varepsilon}{2^{k+1}}
$$

Let $B_{k}:=\left\{u \in \mathbb{D}([0, T], \mathbb{S}): w_{[0, T]}\left(u, \delta_{k}\right) \leq \frac{1}{k}\right\}$ and let $A_{\varepsilon}:=\bigcap_{k=1}^{\infty} B_{k}$. We assert that for each $n \in \mathbb{N}$

$$
\mathbb{P}_{n}\left(A_{\varepsilon}\right) \geq 1-\varepsilon
$$

Indeed, we have the following estimate

$$
\begin{aligned}
\mathbb{P}_{n}\left(\mathbb{D}([0, T], \mathbb{S}) \backslash A_{\varepsilon}\right) & \leq \mathbb{P}_{n}\left(\mathbb{D}([0, T], \mathbb{S}) \backslash \bigcap_{k=1}^{\infty} B_{k}\right)=\mathbb{P}_{n}\left(\bigcup_{k=1}^{\infty}\left(\mathbb{D}([0, T], \mathbb{S}) \backslash B_{k}\right)\right) \\
& \leq \sum_{k=1}^{\infty} \mathbb{P}_{n}\left(\mathbb{D}([0, T], \mathbb{S}) \backslash B_{k}\right) \leq \sum_{k=1}^{\infty} \frac{\varepsilon}{2^{k+1}}=\varepsilon .
\end{aligned}
$$

Thus $\mathbb{P}_{n}\left(A_{\varepsilon}\right) \geq 1-\varepsilon$.

To prove Eq. 88, let us fix $\tilde{\varepsilon}>0$. Directly from the definition of $A_{\varepsilon}$, we infer that $\sup _{u \in A_{\varepsilon}} w_{[0, T]}\left(u, \delta_{k}\right) \leq \frac{1}{k}$ for each $k \in \mathbb{N}$. Choose $k_{0} \in \mathbb{N}$ such that $\frac{1}{k_{0}} \leq \tilde{\varepsilon}$ and let $\delta_{0}:=$ $\delta_{k_{0}}$. Then for every $\delta \leq \delta_{0}$ we obtain

$$
w_{[0, T]}(u, \delta) \leq w_{[0, T]}\left(u, \delta_{k_{0}}\right) \leq \tilde{\varepsilon}
$$

which completes the proof of Eq. 88 and the proof of Lemma.

Now, we recall the Aldous condition which is connected with condition $[\tilde{\mathbf{T}}]$ (see $[19,22]$ and [2]). This condition allows to investigate the modulus for the sequence of stochastic processes by means of stopped processes. 
Definition 5 A sequence $\left(X_{n}\right)_{n \in \mathbb{N}}$ satisfies condition [A] iff

[A] $\forall \varepsilon>0 \quad \forall \eta>0 \quad \exists \delta>0$ such that for every sequence $\left(\tau_{n}\right)_{n \in \mathbb{N}}$ of $\mathbb{F}$-stopping times with $\tau_{n} \leq T$ one has

$$
\sup _{n \in \mathbb{N}} \sup _{0 \leq \theta \leq \delta} \mathbb{P}\left\{\varrho\left(X_{n}\left(\tau_{n}+\theta\right), X_{n}\left(\tau_{n}\right)\right) \geq \eta\right\} \leq \varepsilon .
$$

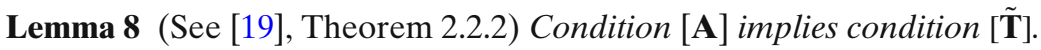

In the following Remark we formulate a certain condition which guaranties that the sequence $\left(X_{n}\right)_{n \in \mathbb{N}}$ satisfies condition [A].

Lemma 9 Let $\left(E,\|\cdot\|_{E}\right)$ be a separable Banach space and let $\left(X_{n}\right)_{n \in \mathbb{N}}$ be a sequence of E-valued random variables. Assume that for every sequence $\left(\tau_{n}\right)_{n \in \mathbb{N}}$ of $\mathbb{F}$-stopping times with $\tau_{n} \leq T$ and for every $n \in \mathbb{N}$ and $\theta \geq 0$ the following condition holds

$$
\mathbb{E}\left[\left(\left\|X_{n}\left(\tau_{n}+\theta\right)-X_{n}\left(\tau_{n}\right)\right\|_{E}^{\alpha}\right] \leq C \theta^{\beta}\right.
$$

for some $\alpha, \beta>0$ and some constant $C>0$. Then the sequence $\left(X_{n}\right)_{n \in \mathbb{N}}$ satisfies condition $[\mathbf{A}]$ in the space $E$.

Proof Let us fix $\varepsilon>0$ and $\eta>0$. By the Chebyshev inequality for every $n \in \mathbb{N}$ and every $\theta>0$ we have

$$
\mathbb{P}\left\{\left\|X_{n}\left(\tau_{n}+\theta\right)-X_{n}\left(\tau_{n}\right)\right\|_{E} \geq \eta\right\} \leq \frac{1}{\eta^{\alpha}} \mathbb{E}\left[\left(\left\|X_{n}\left(\tau_{n}+\theta\right)-X_{n}\left(\tau_{n}\right)\right\|_{E}^{\alpha}\right] \leq \frac{C \theta^{\beta}}{\eta^{\alpha}} .\right.
$$

Let $\delta:=\left(\frac{\eta^{\alpha} \varepsilon}{C}\right)^{\frac{1}{\beta}}$. Let us fix $n \in \mathbb{N}$. Then for every $\theta \in[0, \delta]$ we have the following inequalities

$$
\mathbb{P}\left\{\left\|X_{n}\left(\tau_{n}+\theta\right)-X_{n}\left(\tau_{n}\right)\right\|_{E} \geq \eta\right\} \leq \frac{C \theta^{\beta}}{\eta^{\alpha}} \leq \frac{C}{\eta^{\alpha}}\left[\left(\frac{\eta^{\alpha} \varepsilon}{C}\right)^{\frac{1}{\beta}}\right]^{\beta}=\varepsilon .
$$

Hence

$$
\sup _{0 \leq \theta \leq \delta} \mathbb{P}\left\{\left\|X_{n}\left(\tau_{n}+\theta\right)-X_{n}\left(\tau_{n}\right)\right\|_{E} \geq \eta\right\} \leq \varepsilon .
$$

Since the above inequality holds for every $n \in \mathbb{N}$, one has

$$
\sup _{n \in \mathbb{N}} \sup _{0 \leq \theta \leq \delta} \mathbb{P}\left\{\left\|X_{n}\left(\tau_{n}+\theta\right)-X_{n}\left(\tau_{n}\right)\right\|_{E} \geq \eta\right\} \leq \varepsilon,
$$

i.e. condition $[\mathbf{A}]$ is satisfied. This completes the proof.

\section{A.2 Proof of Corollary 1}

Let $\varepsilon>0$. By the Chebyshev inequality and by (a), we infer that for any $r>0$

$$
\mathbb{P}\left(\sup _{s \in[0, T]}\left|X_{n}(s)\right|_{H}>r\right) \leq \frac{\mathbb{E}\left[\sup _{s \in[0, T]}\left|X_{n}(s)\right|_{H}\right]}{r} \leq \frac{C_{1}}{r} .
$$


Let $R_{1}$ be such that $\frac{C_{1}}{R_{1}} \leq \frac{\varepsilon}{3}$. Then

$$
\mathbb{P}\left(\sup _{s \in[0, T]}\left|X_{n}(s)\right|_{H}>R_{1}\right) \leq \frac{\varepsilon}{3}
$$

Let $B_{1}:=\left\{u \in \mathscr{Z}_{q}: \sup _{s \in[0, T]}|u(s)|_{H} \leq R_{1}\right\}$.

By the Chebyshev inequality and by (b), we infer that for any $r>0$

$$
\mathbb{P}\left(\left\|X_{n}\right\|_{L^{q}(0, T ; V)}>r\right) \leq \frac{\mathbb{E}\left[\left\|X_{n}\right\|_{L^{q}(0, T ; V)}^{q}\right]}{r^{q}} \leq \frac{C_{2}}{r^{q}} .
$$

Let $R_{2}$ be such that $\frac{C_{2}}{R_{2}^{q}} \leq \frac{\varepsilon}{3}$. Then

$$
\mathbb{P}\left(\left\|X_{n}\right\|_{L^{q}(0, T ; V)}>R_{2}\right) \leq \frac{\varepsilon}{3} .
$$

Let $B_{2}:=\left\{u \in \mathscr{Z}_{q}:\|u\|_{L^{q}(0, T ; V)} \leq R_{2}\right\}$.

By Lemmas 7 and 8 there exists a subset $A_{\frac{\varepsilon}{3}} \subset \mathbb{D}\left([0, T], E_{1}\right)$ such that $\tilde{\mathbb{P}}_{n}\left(A_{\frac{\varepsilon}{3}}\right) \geq$ $1-\frac{\varepsilon}{3}$ and

$$
\lim _{\delta \rightarrow 0} \sup _{u \in A_{\frac{\varepsilon}{3}}} w_{[0, T]}(u, \delta)=0 .
$$

It is sufficient to define $K_{\varepsilon}$ as the closure of the set $B_{1} \cap B_{2} \cap A_{\frac{\varepsilon}{3}}$ in $\mathscr{Z}_{q}$. By Theorem $2, K_{\varepsilon}$ is compact in $\mathscr{Z}_{q}$. The proof is thus complete.

\section{Appendix B: The Skorokhod Embedding Theorems}

Let us recall the following Jakubowski's version of the Skorokhod Theorem [18], see also Brzeźniak and Ondreját [10].

Theorem 4 (Theorem 2 in [18]) Let $(\mathscr{X}, \tau)$ be a topological space such that there exists a sequence $\left(f_{m}\right)$ of continuous functions $f_{m}: \mathscr{X} \rightarrow \mathbb{R}$ that separates points of $\mathscr{X}$. Let $\left(X_{n}\right)$ be a sequence of $\mathscr{X}$ valued random variables. Suppose that for every $\varepsilon>0$ there exists a compact subset $K_{\varepsilon} \subset \mathscr{X}$ such that

$$
\sup _{n \in \mathbb{N}} \mathbb{P}\left(\left\{X_{n} \in K_{\varepsilon}\right\}\right)>1-\varepsilon .
$$

Then there exists a subsequence $\left(X_{n_{k}}\right)_{k \in \mathbb{N}}$, a sequence $\left(Y_{k}\right)_{k \in \mathbb{N}}$ of $\mathscr{X}$ valued random variables and an $\mathscr{X}$ valued random variable $Y$ defined on some probability space $(\Omega, \mathscr{F}, \mathbb{P})$ such that

$$
\mathscr{L}\left(X_{n_{k}}\right)=\mathscr{L}\left(Y_{k}\right), \quad k=1,2, \ldots
$$

and forall $\omega \in \Omega$ :

$$
Y_{k}(\omega) \stackrel{\tau}{\longrightarrow} Y(\omega) \quad \text { as } k \rightarrow \infty .
$$

We will use the following version of the Skorokhod Theorem due to Brzeźniak and Hausenblas [6]. 
Theorem 5 (Theorem E.1 in [6]) Let $E_{1}, E_{2}$ be two separable Banach spaces and let $\pi_{i}: E_{1} \times E_{2} \rightarrow E_{i}, i=1,2$, be the projection onto $E_{i}$, i.e.

$$
E_{1} \times E_{2} \ni \chi=\left(\chi_{1}, \chi_{2}\right) \rightarrow \pi_{i}(\chi) \in E_{i} .
$$

Let $(\Omega, \mathscr{F}, \mathbb{P})$ be a probability space and let $\chi_{n}: \Omega \rightarrow E_{1} \times E_{2}, n \in \mathbb{N}$, be a family of random variables such that the sequence $\left\{\mathscr{L}\right.$ aw $\left.\left(\chi_{n}\right), n \in \mathbb{N}\right\}$ is weakly convergent on $E_{1} \times E_{2}$. Finally let us assume that there exists a random variable $\rho: \Omega \rightarrow E_{1}$ such that $\mathscr{L} a w\left(\pi_{1} \circ \chi_{n}\right)=\mathscr{L} a w(\rho), \forall n \in \mathbb{N}$.

Then there exists a probability space $(\bar{\Omega}, \overline{\mathscr{F}}, \overline{\mathbb{P}})$, a family of $E_{1} \times E_{2}$-valued random variables $\left\{\bar{\chi}_{n}, n \in \mathbb{N}\right\}$ on $(\bar{\Omega}, \overline{\mathscr{F}}, \overline{\mathbb{P}})$ and a random variable $\chi_{*}: \bar{\Omega} \rightarrow E_{1} \times E_{2}$ such that

(i) $\mathscr{L} a w\left(\bar{\chi}_{n}\right)=\mathscr{L} a w\left(\chi_{n}\right), \forall n \in \mathbb{N}$;

(ii) $\bar{\chi}_{n} \rightarrow \chi_{*}$ in $E_{1} \times E_{2}$ a.s.;

(iii) $\pi_{1} \circ \bar{\chi}_{n}(\bar{\omega})=\pi_{1} \circ \chi_{*}(\bar{\omega})$ for all $\bar{\omega} \in \bar{\Omega}$.

Remark Theorem 5 remains true if we substitute the Banach spaces $E_{1}, E_{2}$ by the separable complete metric spaces.

Using the ideas due to Jakubowski [18], we can proof the following generalization of Theorem 5 to the case of nonmetric spaces. Let us notice that in comparison to Theorem 5 we will assume that the sequence $\left\{\mathscr{L} a w\left(\chi_{n}\right), n \in \mathbb{N}\right\}$ is tight. The assumption of the weak convergence of $\left\{\mathscr{L} a w\left(\chi_{n}\right), n \in \mathbb{N}\right\}$ is not sufficient in the case of nonmetric spaces, see [18].

Corollary 2 (Corollary 5.3 in [25]) Let $\mathscr{X}_{1}$ be a separable complete metric space and let $\mathscr{X}_{2}$ be a topological space such that there exists a sequence $\left\{f_{l}\right\}_{l \in \mathbb{N}}$ of continuous functions $f_{\imath}: \mathscr{X}_{2} \rightarrow \mathbb{R}$ separating points of $\mathscr{X}_{2}$. Let $\mathscr{X}:=\mathscr{X}_{1} \times \mathscr{X}_{2}$ with the Tykhonoff topology induced by the projections

$$
\pi_{i}: \mathscr{X}_{1} \times \mathscr{X}_{2} \rightarrow \mathscr{X}_{i}, \quad i=1,2 .
$$

Let $(\Omega, \mathscr{F}, \mathbb{P})$ be a probability space and let $\chi_{n}: \Omega \rightarrow \mathscr{X}_{1} \times \mathscr{X}_{2}, n \in \mathbb{N}$, be a family of random variables such that the sequence $\left\{\mathscr{L}\right.$ aw $\left.\left(\chi_{n}\right), n \in \mathbb{N}\right\}$ is tight on $\mathscr{X}_{1} \times \mathscr{X}_{2}$. Finally let us assume that there exists a random variable $\rho: \Omega \rightarrow \mathscr{X}_{1}$ such that $\mathscr{L}$ aw $\left(\pi_{1} \circ \chi_{n}\right)=\mathscr{L}$ aw $(\rho)$ for all $n \in \mathbb{N}$.

Then there exists a subsequence $\left(\chi_{n_{k}}\right)_{k \in \mathbb{N}}$, a probability space $(\bar{\Omega}, \overline{\mathscr{F}}, \overline{\mathbb{P}})$, a family of $\mathscr{X}_{1} \times \mathscr{X}_{2}$-valued random variables $\left\{\bar{\chi}_{k}, k \in \mathbb{N}\right\}$ on $(\bar{\Omega}, \overline{\mathscr{F}}, \overline{\mathbb{P}})$ and a random variable $\chi_{*}: \bar{\Omega} \rightarrow \mathscr{X}_{1} \times \mathscr{X}_{2}$ such that

(i) $\mathscr{L} a w\left(\bar{\chi}_{k}\right)=\mathscr{L}$ aw $\left(\chi_{n_{k}}\right)$ for all $k \in \mathbb{N}$;

(ii) $\bar{\chi}_{k} \rightarrow \chi_{*}$ in $\mathscr{X}_{1} \times \mathscr{X}_{2}$ a.s. as $k \rightarrow \infty$;

(iii) $\pi_{1} \circ \bar{\chi}_{k}(\bar{\omega})=\pi_{1} \circ \chi_{*}(\bar{\omega})$ for all $\bar{\omega} \in \bar{\Omega}$.

For the convenience of the reader we recall the proof.

Proof Using the ideas due to Jakubowski [18], the proof can be reduced to Theorem 5. Let us denote

$$
\chi_{n}=\left(\chi_{n}^{1}, \chi_{n}^{2}\right)
$$


where $\chi_{n}^{i}:=\pi_{i} \circ \chi_{n}, i=1,2$. Since the sequence $\left\{\mathscr{L} a \mathrm{w}\left(\chi_{n}\right), n \in \mathbb{N}\right\}$ is tight on $\mathscr{X}_{1} \times$ $\mathscr{X}_{2}$, we infer that the sequence $\left\{\mathscr{L} a \mathrm{w}\left(\chi_{n}^{2}\right), n \in \mathbb{N}\right\}$ is tight on $\mathscr{X}_{2}$. Let $K_{m} \subset \mathscr{X}_{2}$ be compact subsets such that $K_{m} \subset K_{m+1}, m=1,2, \ldots$ and

$$
\sup _{n \in \mathbb{N}} \mathbb{P}\left(\left\{\chi_{n}^{2} \in K_{m}\right\}\right)>1-\frac{1}{m} .
$$

Let us consider the mapping $\tilde{f}: \mathscr{X}_{2} \rightarrow \mathbb{R}^{\mathbb{N}}$ defined by

$$
\begin{gathered}
\tilde{f}(z):=\left(f_{1}(z), f_{2}(z), \ldots\right)=\left(f_{\iota}(z)\right)_{\iota \in \mathbb{N}}, \quad z \in \mathscr{X}_{2} . \\
\tilde{\mu}_{n}:=\mathscr{L}\left(\tilde{f}\left(\chi_{n}^{2}\right)\right) \quad \text { and } \quad \tilde{K}_{m}:=\tilde{f}\left(K_{m}\right) .
\end{gathered}
$$

On the set $\mathbb{R}^{\mathbb{N}}$ let us define the function

$$
\begin{aligned}
& \Phi(y):=\min \left\{m: \quad y \in \tilde{K}_{m}\right\} \quad \text { if } y \in \bigcup_{m=1}^{\infty} \tilde{K}_{m} \\
& \Phi(y)=+\infty \quad \text { otherwise. }
\end{aligned}
$$

Function $\Phi: \mathbb{R}^{\mathbb{N}} \rightarrow \mathbb{N}$ is lower semicontinuous, i.e.: if $y_{n} \rightarrow y_{0}$ in $\mathbb{R}^{\mathbb{N}}$, then

$$
\liminf _{n \rightarrow \infty} \Phi\left(y_{n}\right) \geq \Phi\left(y_{0}\right)
$$

From Eq. 90 it follows that

- $\Phi<\infty\left(\tilde{\mu}_{n}\right.$-p.p. $)$ for all $n \in \mathbb{N}$

- $\quad$ and $\left(\tilde{\mu}_{n} \circ \Phi^{-1}\right)$ is a tight sequence of laws on $\mathbb{N}$.

Furthermore, the sequence of laws $\left\{\mathscr{L} a w\left(\tilde{f} \circ \chi_{n}^{2}, \Phi \circ \tilde{f} \circ \chi_{n}^{2}\right), n \in \mathbb{N}\right\}$ is tight on $\mathbb{R}^{\mathbb{N}} \times \mathbb{N}$.

Let us consider the product space $\mathscr{X}_{1} \times\left(\mathbb{R}^{\mathbb{N}} \times \mathbb{N}\right)$ and let $P_{1}:=\mathscr{X}_{1} \times\left(\mathbb{R}^{\mathbb{N}} \times\right.$ $\mathbb{N}) \rightarrow \mathscr{X}_{1}$ be the projection onto $\mathscr{X}_{1}$ and $P_{2}:=\mathscr{X}_{1} \times\left(\mathbb{R}^{\mathbb{N}} \times \mathbb{N}\right) \rightarrow \mathbb{R}^{\mathbb{N}} \times \mathbb{N}$ be the projection onto $\mathbb{R}^{\mathbb{N}} \times \mathbb{N}$. Moreover let $\xi_{n}, n \in \mathbb{N}$, be $\mathscr{X}_{1} \times\left(\mathbb{R}^{\mathbb{N}} \times \mathbb{N}\right)$-valued random variables defined by

$$
\xi_{n}:=\left(\xi_{n}^{1}, \xi_{n}^{2}\right): \Omega \rightarrow \mathscr{X}_{1} \times\left(\mathbb{R}^{\mathbb{N}} \times \mathbb{N}\right)
$$

where

$$
\xi_{n}^{1}:=\chi_{n}^{1} \quad \text { and } \quad \xi_{n}^{2}:=\left(\tilde{f} \circ \chi_{n}^{2}, \Phi \circ \tilde{f} \circ \chi_{n}^{2}\right), \quad n \in \mathbb{N} .
$$

Remark that the sequence of laws $\left\{\mathscr{L} a w\left(\xi_{n}\right), n \in \mathbb{N}\right\}$ is tight on $\mathscr{X}_{1} \times\left(\mathbb{R}^{\mathbb{N}} \times \mathbb{N}\right)$. By the Prokhorov Theorem we can choose a subsequence $\left(n_{k}\right)_{k \in \mathbb{N}}$ such that $\left\{\mathscr{L} a w\left(\xi_{n_{k}}\right), k \in \mathbb{N}\right\}$ is weakly convergent on $\mathscr{X}_{1} \times\left(\mathbb{R}^{\mathbb{N}} \times \mathbb{N}\right)$. Thus the subsequence $\left(\xi_{n_{k}}\right)_{k \in \mathbb{N}}$ satisfies the assumption of Theorem 5. Hence there exists a probability space $(\bar{\Omega}, \overline{\mathscr{F}}, \overline{\mathbb{P}})$, a family of $\mathscr{X}_{1} \times\left(\mathbb{R}^{\mathbb{N}} \times \mathbb{N}\right)$-valued random variables $\left\{\bar{\xi}_{k}, k \in \mathbb{N}\right\}$ on $(\bar{\Omega}, \overline{\mathscr{F}}, \overline{\mathbb{P}})$ and a random variable $\xi_{*}: \bar{\Omega} \rightarrow \mathscr{X}_{1} \times\left(\mathbb{R}^{\mathbb{N}} \times \mathbb{N}\right)$ such that

(i) $\mathscr{L} a w\left(\bar{\xi}_{k}\right)=\mathscr{L} a w\left(\xi_{n_{k}}\right)$ for all $k \in \mathbb{N}$;

(ii) $\bar{\xi}_{k} \rightarrow \xi_{*}$ in $\mathscr{X}_{1} \times\left(\mathbb{R}^{\mathbb{N}} \times \mathbb{N}\right)$ a.s. as $k \rightarrow \infty$;

(iii) $P_{1} \circ \bar{\xi}_{k}(\bar{\omega})=P_{1} \circ \xi_{*}(\bar{\omega})$ for all $\bar{\omega} \in \bar{\Omega}$. 
Let us put

$$
\bar{\chi}_{k}^{1}:=P_{1} \circ \bar{\xi}_{k}, \quad k \in \mathbb{N} \text {. }
$$

Notice that $\left(P_{2} \circ \bar{\xi}_{k}\right)_{k \in \mathbb{N}}$ is the Skorokhod representation for the sequence $\left(\tilde{f} \circ \chi_{n_{k}}^{2}, \Phi \circ \tilde{f} \circ \chi_{n_{k}}^{2}\right)_{k \in \mathbb{N}}$. Let $P_{2} \circ \bar{\xi}_{k}=\left(\eta_{k}^{1}, \eta_{k}^{2}\right)$, where $\eta_{k}^{1}: \bar{\Omega} \rightarrow \mathbb{R}^{\mathbb{N}}$ and $\eta_{k}^{2}: \bar{\Omega} \rightarrow \mathbb{N}$, $k \in \mathbb{N}$ and let $P_{2} \circ \xi_{*}=\left(\eta_{*}^{1}, \eta_{*}^{2}\right)$, where $\eta_{*}^{1}: \bar{\Omega} \rightarrow \mathbb{R}^{\mathbb{N}}$ and $\eta_{*}^{2}: \bar{\Omega} \rightarrow \mathbb{N}$. In the same way as in the proof of Lemma 1 in [18], we can prove that $\eta_{k}^{2}=\Phi\left(\eta_{k}^{1}\right)$, $\overline{\mathbb{P}}$-a.s., $k \in \mathbb{N}$. Since $\eta_{*}^{2}<\infty \overline{\mathbb{P}}$-a.s., we have

$$
\sup _{k \in \mathbb{N}} \Phi\left(\eta_{k}^{1}\right)<\infty \quad \overline{\mathbb{P}} \text {-a.s. }
$$

Thus for $\overline{\mathbb{P}}$-almost all $\omega \in \bar{\Omega}$ the values $\eta_{k}^{1}(\omega)$ belong to the $\sigma$-compact subspace $\bigcup_{m=1}^{\infty} \tilde{K}_{m}=\tilde{f}\left(\bigcup_{m=1}^{\infty} K_{m}\right)$. Since $\tilde{f}$ restricted to $\sigma$-compact subspace is a measurable homeomorphism, we can define

$$
\bar{\chi}_{k}^{2}:=\tilde{f}^{-1}\left(\eta_{k}^{1}\right), \quad k \in \mathbb{N} .
$$

Finally $\bar{\chi}_{k}$ is defined by

$$
\bar{\chi}_{k}:=\left(\bar{\chi}_{k}^{1}, \bar{\chi}_{k}^{2}\right), \quad k \in \mathbb{N} \text {. }
$$

This completes the proof.

In Section 5 we use Corollary 2 for the space

$$
\mathscr{X}_{2}:=\mathscr{Z}:=L_{w}^{2}(0, T ; V) \cap L^{2}\left(0, T ; H_{\mathrm{loc}}\right) \cap \mathbb{D}\left([0, T] ; U^{\prime}\right) \cap \mathbb{D}\left([0, T] ; H_{w}\right) .
$$

So, in the following Remark we will discuss the problem of existence of the countable family of real valued continuous mappings defined on $\mathscr{Z}$ and separating points of this space.

\section{Remark 2}

(1) Since $L^{2}\left(0, T ; H_{\text {loc }}\right)$ and $\mathbb{D}\left([0, T] ; U^{\prime}\right)$ are separable and completely metrizable spaces, we infer that on each of these spaces there exists a countable family of continuous real valued mappings separating points, see [3], exposé 8.

(2) For the space $L_{w}^{2}(0, T ; V)$ it is sufficient to put

$$
f_{m}(u):=\int_{0}^{T}\left(\left(u(t) v_{n}(t) d t \in \mathbb{R}, \quad u \in L^{2}(0, T ; V), \quad m \in \mathbb{N},\right.\right.
$$

where $\left\{v_{m}, m \in \mathbb{N}\right\}$ is a dense subset of $L^{2}(0, T ; V)$. Then $\left(f_{m}\right)_{m \in \mathbb{N}}$ is a sequence of continuous real valued mappings separating points of the space $L_{w}^{2}(0, T ; V)$.

(3) Let $H_{0} \subset H$ be a countable and dense subset of $H$. Then by Eq. 25 for each $h \in H_{0}$ the mapping

$$
\mathbb{D}\left([0, T] ; H_{w}\right) \ni u \mapsto(u(\cdot) \mid h)_{H} \in \mathbb{D}([0, T] ; \mathbb{R})
$$

is continuous. Since $\mathbb{D}([0, T] ; \mathbb{R})$ is a separable complete metric space, there exists a sequence $\left(g_{l}\right)_{l \in \mathbb{N}}$ of real valued continuous functions defined on 
$\mathbb{D}([0, T] ; \mathbb{R})$ separating points of this space. Then the mappings $f_{h, l}, h \in H_{0}$, $l \in \mathbb{N}$ defined by

$$
f_{h, l}(u):=g_{l}\left((u(\cdot) \mid h)_{H}\right), \quad u \in \mathbb{D}\left([0, T] ; H_{w}\right),
$$

form a countable family of continuous mappings on $\mathbb{D}\left([0, T] ; H_{w}\right)$ separating points of this space.

\section{Appendix C: Some Auxilliary Results from Functional Analysis}

The following result can be found in Holly and Wiciak, [16]. We recall it together with the proof.

Lemma 10 (see Lemma 2.5, p.99 in [16]) Consider a separable Banach space $\Phi$ having the following property

$$
\text { there exists a Hilbert space } \mathbb{H} \text { such that } \Phi \subset \mathbb{H} \text { continuously. }
$$

Then there exists a Hilbert space $\left(\mathscr{H},(\cdot \mid \cdot)_{\mathscr{H}}\right)$ such that $\mathscr{H} \subset \phi, \mathscr{H}$ is dense in $\Phi$ and the embedding $\mathscr{H} \hookrightarrow \Phi$ is compact.

Proof Without loss of generality we can assume that $\operatorname{dim} \Phi=\infty$ and $\Phi$ is dense in $\mathbb{H}$. Since $\Phi$ is separable, there exists a sequence $\left(\varphi_{n}\right)_{n \in \mathbb{N}} \subset \Phi$ linearly dense in $\Phi$. Since $\Phi$ is dense in $\mathbb{H}$ and the embedding $\Phi \hookrightarrow \mathbb{H}$ is continuous, the subspace $\operatorname{span}\left\{\varphi_{1}, \varphi_{2}, \ldots\right\}$ is dense in $\mathbb{H}$. After the orthonormalisation of $\left(\varphi_{n}\right)$ in the Hilbert space $\left(\mathbb{H},(\cdot \mid \cdot)_{\mathbb{H}}\right)$ we obtain an orthonormal basis $\left(h_{n}\right)$ of this space. Furthermore, the sequence $\left(h_{n}\right)$ is linearly dense in $\Phi$. Since the natural embedding $\iota: \Phi \hookrightarrow \mathbb{H}$ is continuous, we infer that

$$
1=\left|h_{n}\right|_{\mathbb{H}}=\left|\iota\left(h_{n}\right)\right|_{\mathbb{H}} \leq|\iota| \cdot\left|h_{n}\right|_{\Phi}
$$

and

$$
\frac{1}{\left|h_{n}\right|_{\Phi}} \leq|\iota| \quad \text { for all } n \in \mathbb{N}
$$

Let us take $\eta_{0} \in(0,1)$ and define inductively a sequence $\left(\eta_{n}\right)_{n \in \mathbb{N}}$ by

$$
\eta_{n}:=\frac{\eta_{n-1}+1}{2}, \quad n=1,2, \ldots
$$

The sequence $\left(\eta_{n}\right)$ is strongly increasing and $\lim _{\rightarrow \infty} \eta_{n}=1$. Let us define a sequence $\left(r_{n}\right)_{n \in \mathbb{N}}$ by

$$
r_{n}:=\frac{1-\eta_{n}}{2\left|h_{n}\right|_{\Phi}}>0, \quad n=1,2, \ldots
$$

Obviously $\lim _{\rightarrow \infty} r_{n}=0$. Let us consider the set

$$
\mathscr{H}:=\left\{x \in \mathbb{H}: \quad \sum_{n=1}^{\infty} \frac{1}{r_{n}^{2}} \cdot\left|\left(x \mid h_{n}\right)_{\mathbb{H}}\right|^{2}<\infty\right\}
$$


and the Hilbert space $L_{\mu}^{2}\left(\mathbb{N}^{*}, \mathbb{R}\right)$, where $\mu: 2^{\mathbb{N}^{*}} \rightarrow[0, \infty]$ is the measure given by the formula

$$
\mu(M):=\sum_{n \in M} \frac{1}{r_{n}^{2}}, \quad M \subset \mathbb{N}^{*}
$$

The linear operator

$$
l: L_{\mu}^{2}\left(\mathbb{N}^{*}, \mathbb{R}\right) \ni \xi \mapsto \sum_{n=1}^{\infty} \xi_{n} h_{n} \in \mathbb{H}
$$

is well defined. Moreover, $l$ is an injection and hence we may introduce the following inner product

$$
(\cdot \mid \cdot)_{\mathscr{H}}:=(\cdot \mid \cdot)_{L^{2}} \stackrel{\circ}{ } l^{-1}: \mathscr{H} \times \mathscr{H} \ni(x, y) \mapsto\left(l^{-1} x \mid l^{-1} y\right)_{L^{2}} \in \mathbb{R} .
$$

Now, $l$ is an isometry onto the pre-Hilbert space $\left(\mathscr{H},(\cdot \mid \cdot)_{\mathscr{H}}\right)$ and consequently $\mathscr{H}$ is $(\cdot \mid \cdot)_{\mathscr{H}}$-complete. Let us notice that for all $x, y \in \mathscr{H}$

$$
(x \mid y)_{\mathscr{H}}=\sum_{n=1}^{\infty} \frac{1}{r_{n}^{2}} \cdot\left(x \mid h_{n}\right)_{\mathbb{H}}\left(y \mid h_{n}\right)_{\mathbb{H}}, \quad|x|_{\mathscr{H}}^{2}=\sum_{n=1}^{\infty} \frac{1}{r_{n}^{2}} \cdot\left|\left(x \mid h_{n}\right)_{\mathbb{H}}\right|^{2}
$$

We will show that $\mathscr{H} \subset \Phi$ continuously. Indeed, let $x \in \mathscr{H},|x|_{\mathscr{H}} \leq 1$. Then for each $i \in \mathbb{N}$

$$
\left|\left(x \mid h_{i}\right) h_{i}\right|_{\Phi}=\left|\left(x \mid h_{i}\right)\right| \cdot\left|h_{i}\right|_{\Phi} \leq r_{i}\left|h_{i}\right|_{\Phi}=\frac{1-\eta_{i-1}}{2\left|h_{i}\right|_{\Phi}}\left|h_{i}\right|_{\Phi}=\frac{1-\eta_{i-1}}{2}=\eta_{i}-\eta_{i-1} .
$$

Thus, for any $k, n \in \mathbb{N}, k<n$, we have the following estimate

$$
\left|\sum_{i=k+1}^{n}\left(x \mid h_{i}\right) h_{i}\right|_{\Phi} \leq \sum_{i=k+1}^{n}\left(\eta_{i}-\eta_{i-1}\right)=\eta_{n}-\eta_{k} .
$$

Since in particular, the sequence $\left(s_{n}:=\sum_{i=1}^{n}\left(x \mid h_{i}\right) h_{i}\right)$ is Cauchy in the Banach space $\left(\Phi,|\cdot|_{\Phi}\right)$, there exists $\varphi \in \Phi$ such that $\lim _{n}\left|s_{n}-\varphi\right|_{\Phi}=0$. On the other hand, $s_{n}=$ $\sum_{i=1}^{n}\left(x \mid h_{i}\right) h_{i} \rightarrow x$ in $\mathbb{H}$. Thus by the uniqueness of the limit $\varphi=x \in \Phi$ and

$$
\sum_{i=1}^{n}\left(x \mid h_{i}\right) h_{i} \rightarrow x \quad \text { in } \Phi .
$$

Moreover,

$$
|x|_{\Phi} \stackrel{\infty \leftarrow n}{\longleftarrow}\left|s_{n}\right|_{\Phi} \leq \eta_{n}-\eta_{0} \stackrel{n \rightarrow \infty}{\longrightarrow} 1-\eta_{0} .
$$

Thus $\mathscr{H} \subset \Phi$ continuously (with the norm of the embedding not exceeding $1-\eta_{0}$ ). We will show that the embedding $j: \mathscr{H} \hookrightarrow \Phi$ is compact. It is sufficient to prove that the ball $Z:=\left\{x \in \mathscr{H}:|x|_{\mathscr{H}} \leq 1\right\}$ is relatively compact in $\left(\Phi,|\cdot|_{\Phi}\right)$. According to the Hausdorff Theorem it is sufficient to find (for every fixed $\varepsilon$ ) an $\varepsilon$-net of the set $j(Z)$.

Since $\lim _{n \rightarrow \infty} \eta_{n}=1$, there exists $n \in \mathbb{N}$ such that $1-\eta_{n} \leq \frac{\varepsilon}{2}$. The linear operator

$$
S_{n}: \mathscr{H} \ni x \mapsto \sum_{i=1}^{n}\left(x \mid h_{i}\right) h_{i} \in \Phi
$$


being finite-dimensional is compact. Therefore $S_{n}(Z)$ is relatively compact in $\left(\Phi,|\cdot|_{\Phi}\right)$ and consequently there is a finite subset $F \subset \Phi$ such that $S_{n}(Z) \subset$ $\bigcup_{\varphi \in Z} \mathbb{B}_{\Phi}\left(\varphi, \frac{\varepsilon}{2}\right)$.

We will show that the set $F$ is the $\varepsilon$-net for $j(Z)$. Indeed, let $x \in Z$. Then $S_{N}(x) \rightarrow$ $x$ in $\left(\Phi,|\cdot|_{\Phi}\right)$ and

$$
\left|x-S_{n}(x)\right|_{\Phi} \stackrel{\infty \leftarrow N}{\longleftarrow}\left|S_{N}(x)-S_{n}(x)\right|_{\Phi} \leq \eta_{N}-\eta_{n} \stackrel{N \rightarrow \infty}{\longrightarrow} 1-\eta_{n} \leq \frac{\varepsilon}{2} .
$$

On the other hand, $S_{n}(x) \in S_{n}(Z)$, so, there is $\varphi \in F$ such that $S_{n}(x) \in \mathbb{B}_{\Phi}\left(\varphi, \frac{\varepsilon}{2}\right)$. Finally,

$$
|x-\varphi|_{\Phi} \leq\left|x-S_{n}(x)\right|_{\Phi}+\left|S_{n}(x)-\varphi\right|_{\Phi} \leq \frac{\varepsilon}{2}+\frac{\varepsilon}{2}=\varepsilon,
$$

i.e. $x \in \mathbb{B}_{\Phi}(\varphi, \varepsilon)$. Thus

$$
Z \subset \bigcup_{\varphi \in \Phi} \mathbb{B}_{\Phi}(\varphi, \varepsilon)
$$

The proof is thus complete.

\section{Appendix D: Proof of Lemma 6}

Proof Assume first that $\psi \in \mathscr{V}$. Then there exists $R>0$ such that supp $\psi$ is a compact subset of $\mathscr{O}_{R}$. Then, using the integration by parts formula, we infer that for every $v, w \in H$

$$
\begin{aligned}
& \mid\left\langle B(v, w)|\psi|=\left|\int_{\mathscr{O}_{R}}(v \cdot \nabla \psi) w d x\right|\right. \\
& \leq\|u\|_{L^{2}\left(\mathscr{O}_{R}\right)}\|w\|_{L^{2}\left(\mathscr{O}_{R}\right)}\|\nabla \psi\|_{L^{\infty}\left(\mathscr{O}_{R}\right)} \leq C|u|_{H_{\mathscr{O}_{R}}}|w|_{H_{\mathscr{O}_{R}}}\|\psi\|_{V_{m}} .
\end{aligned}
$$

We have $B\left(u_{n}, u_{n}\right)-B(u, u)=B\left(u_{n}-u, u_{n}\right)+B\left(u, u_{n}-u\right)$. Thus, using the estimate (92) and the Hőlder inequality, we obtain

$$
\begin{aligned}
& \left|\int_{0}^{t}\left\langle B\left(u_{n}(s), u_{n}(s)\right) \mid \psi\right\rangle d s-\int_{0}^{t}\langle B(u(s), u(s)) \mid \psi\rangle d s\right| \\
& \leq\left|\int_{0}^{t}\left\langle B\left(u_{n}(s)-u(s), u_{n}(s)\right) \mid \psi\right\rangle d s\right|+\left|\int_{0}^{t}\left\langle B\left(u(s), u_{n}(s)-u(s)\right) \mid \psi\right\rangle d s\right| \\
& \leq C \cdot\left\|u_{n}-u\right\|_{L^{2}\left(0, T ; H_{\mathscr{O}_{R}}\right)}\left(\left\|u_{n}\right\|_{L^{2}\left(0, T ; H_{\mathscr{O}_{R}}\right)}+\|u\|_{L^{2}\left(0, T ; H_{\mathscr{O}_{R}}\right)}\right)\|\psi\|_{V_{m}},
\end{aligned}
$$

where $C$ stands for some positive constant. Since $u_{n} \rightarrow u$ in $L^{2}\left(0, T ; H_{\text {loc }}\right)$, we infer that for all $\psi \in \mathscr{V}$

$$
\lim _{n \rightarrow \infty} \int_{0}^{t}\left\langle B\left(u_{n}(s)\right) \mid \psi\right\rangle d s=\int_{0}^{t}\langle B(u(s)) \mid \psi\rangle d s .
$$


If $\psi \in V_{m}$ then for every $\varepsilon>0$ there exists $\psi_{\varepsilon} \in \mathscr{V}$ such that $\left\|\psi-\psi_{\varepsilon}\right\|_{V_{m}} \leq \varepsilon$. Then

$$
\begin{aligned}
\mid\langle & B\left(u_{n}(s)\right)-B(u(s))|\psi\rangle|=|\left\langle B\left(u_{n}(s)\right)-B(u(s)) \mid \psi-\psi_{\varepsilon}\right\rangle \mid \\
& +\left|\left\langle B\left(u_{n}(s)\right)-B(u(s)) \mid \psi_{\varepsilon}\right\rangle\right| \\
\leq & \left(\left|B\left(u_{n}(s)\right)\right|_{V_{m}^{\prime}}+|B(u(s))|_{V_{m}^{\prime}}\right) \cdot\left\|\psi-\psi_{\varepsilon}\right\|_{V_{m}} \\
& +\left|\left\langle B\left(u_{n}(s)\right)-B(u(s)) \mid \psi_{\varepsilon}\right\rangle\right| \\
\leq & \varepsilon\left(\left|u_{n}(s)\right|_{H}^{2}+|u(s)|_{H}^{2}\right)+\left|\left\langle B\left(u_{n}(s)\right)-B(u(s)) \mid \psi_{\varepsilon}\right\rangle\right| .
\end{aligned}
$$

Hence

$$
\begin{aligned}
& \left|\int_{0}^{t}\left\langle B\left(u_{n}(s)\right)-B(u(s)) \mid \psi\right\rangle d s\right| \\
& \leq \varepsilon \int_{0}^{t}\left(\left|u_{n}(s)\right|_{H}^{2}+|u(s)|_{H}^{2}\right) d s+\left|\int_{0}^{t}\left\langle B\left(u_{n}(s)\right)-B(u(s)) \mid \psi_{\varepsilon}\right\rangle d s\right| \\
& \leq \varepsilon \cdot\left(\sup _{n \geq 1}\left\|u_{n}\right\|_{L^{2}(0, T ; H)}^{2}+\|u\|_{L^{2}(0, T ; H)}^{2}\right)+\left|\int_{0}^{t}\left\langle B\left(u_{n}(s)\right)-B(u(s)) \mid \psi_{\varepsilon}\right\rangle d s\right| .
\end{aligned}
$$

Passing to the upper limit as $n \rightarrow \infty$, we obtain

$$
\lim \sup _{n \rightarrow \infty}\left|\int_{0}^{t}\left\langle B\left(u_{n}(s)\right)-B(u(s)) \mid \psi\right\rangle d s\right| \leq M \varepsilon,
$$

where $M:=\sup _{n \geq 1}\left\|u_{n}\right\|_{L^{2}(0, T ; H)}^{2}+\|u\|_{L^{2}(0, T ; H)}^{2}<\infty$. Since $\varepsilon>0$ is arbitrary, we infer that Eq. 93 holds for all $\psi \in V_{m}$. The proof of the Lemma 6 is thus complete.

\section{Appendix E: Proof of Lemma 2}

To prove that $u_{n} \in \mathbb{D}\left([0, T] ; H_{w}\right)$, it is sufficient to show that for every $h \in H$ the real-valued functions $\left(u_{n}(\cdot) \mid h\right)$ are càdlàg on $[0, T]$, i.e. are right continuous and have left limits at every $t \in[0, T]$. Let us fix $n \in \mathbb{N}$ and $t_{0} \in[0, T]$ and let us assume that $h \in U$. Since $u_{n} \in \mathbb{D}\left([0, T] ; U^{\prime}\right)$, there exists $a \in U^{\prime}$ such that

$$
\lim _{t \rightarrow t_{0}^{-}}\left\|u_{n}(t)-a\right\|_{U^{\prime}}=0 .
$$

In fact, $a \in H$. Indeed, by assumption (i) $u_{n}([0, T]) \subset H$ and

$$
\sup _{s \in[0, T]}\left|u_{n}(s)\right|_{H} \leq r .
$$

Let $\left(t_{k}\right)_{k \in \mathbb{N}} \subset[0, T]$ be a sequence convergent to $t_{0}^{-}$. Since $\left|u_{n}\left(t_{k}\right)\right|_{H} \leq r$, by the Banach-Alaoglu Theorem there exists a subsequence convergent weakly in $H$ to some $b \in H$, i.e. there exists $\left(t_{k_{l}}\right)_{l \in \mathbb{N}}$ such that $u_{n}\left(t_{k_{l}}\right) \rightarrow b$ weakly in $H$ as $l \rightarrow \infty$. Since the embedding $H \hookrightarrow U^{\prime}$ is continuous, we infer that

$$
u_{n}\left(t_{k_{l}}\right) \rightarrow b \quad \text { weakly in } U^{\prime} \text { as } l \rightarrow \infty .
$$

On the other hand, by Eq. 94

$$
u_{n}\left(t_{k_{l}}\right) \rightarrow a \quad \text { in } U^{\prime} \text { as } l \rightarrow \infty .
$$


Hence $a=b \in H$.

We have

$$
\left|\left(u_{n}(t)-a \mid h\right)_{H}\right|=\left|\left\langle u_{n}(t)-a \mid h\right\rangle\right| \leq\left\|u_{n}(t)-a\right\|_{U^{\prime}} \cdot\|h\|_{U} .
$$

By Eqs. 94 and 95 we infer that $\lim _{t \rightarrow t_{0}}\left(u_{n}(t)-a \mid h\right)_{H}=0$. Now, let $h \in H$ and let $\varepsilon>0$. Since $U$ is dense in $H$, there exists $h_{\varepsilon} \in U$ such that $\left|h-h_{\varepsilon}\right|_{H} \leq \varepsilon$. We have the following inequalities

$$
\begin{aligned}
\left|\left(u_{n}(t)-a \mid h\right)_{H}\right| & \leq\left|\left(u_{n}(t)-a \mid h-h_{\varepsilon}\right)_{H}\right|+\left|\left(u_{n}(t)-a \mid h_{\varepsilon}\right)_{H}\right| \\
& \leq\left|u_{n}(t)-a\right|_{H}\left|h-h_{\varepsilon}\right|_{H}+\left|\left(u_{n}(t)-a \mid h_{\varepsilon}\right)_{H}\right| \\
& \leq 2 \varepsilon\left\|u_{n}\right\|_{L^{\infty}(0, T ; H)}+\left|\left(u_{n}(t)-a \mid h_{\varepsilon}\right)_{H}\right| \\
& \leq 2 \varepsilon r+\left|\left(u_{n}(t)-a \mid h_{\varepsilon}\right)_{H}\right| .
\end{aligned}
$$

Passing to the upper limit as $t \rightarrow t_{0}^{-}$, we obtain

$$
\lim \sup _{t \rightarrow t_{0}^{-}}\left|\left(u_{n}(t)-a \mid h\right)_{H}\right| \leq 2 \varepsilon r
$$

Since $\varepsilon$ was chosen in an arbitrary way, we infer that

$$
\lim _{t \rightarrow t_{0}^{-}}\left(u_{n}(t)-a \mid h\right)_{H}=0 .
$$

The proof of right continuity of $u_{n}$ is analogous.

We claim that

$$
u_{n} \rightarrow u \quad \text { in } \mathbb{D}\left([0, T] ; \mathbb{B}_{w}\right) \quad \text { as } \quad n \rightarrow \infty
$$

i.e. that for all $h \in H$

$$
\left(u_{n} \mid h\right)_{H} \rightarrow(u \mid h)_{H} \quad \text { in } \quad \mathbb{D}([0, T] ; \mathbb{R}) .
$$

By (ii) and Remark 1 there exists a sequence $\left(\lambda_{n}\right) \subset \Lambda_{T}$ converging to identity uniformly on $[0, T]$ and such that

$$
u_{n} \circ \lambda_{n} \rightarrow u \text { in } U^{\prime}
$$

uniformly on $[0, T]$. We will prove that for all $h \in H$

$$
\left(u_{n} \circ \lambda_{n} \mid h\right)_{H} \rightarrow(u \mid h)_{H} \quad \text { in } \quad \mathbb{R}
$$

uniformly on $[0, T]$.

Indeed, let us first fix $h \in U$. Then for all $s \in[0, T]$ we have

$$
\left|\left(u_{n} \circ \lambda_{n}(s)-u(s) \mid h\right)_{H}\right|=\left|\left\langle u_{n} \circ \lambda_{n}(s)-u(s)\right| h\right) \mid \leq\left\|u_{n} \circ \lambda_{n}(s)-u(s)\right\|_{U^{\prime}}\|h\|_{U} .
$$

By Remark 1

$$
\sup _{s \in[0, T]}\left|\left\langle u_{n} \circ \lambda_{n}(s)-u(s) \mid h\right\rangle\right| \leq \sup _{s \in[0, T]}\left\|u_{n} \circ \lambda_{n}(s)-u(s)\right\|_{U^{\prime}} \cdot\|h\|_{U} \rightarrow 0
$$


as $n \rightarrow \infty$. Moreover, since $U$ is dense in $H$, the desired convergence holds for all $h \in H$. Indeed, let us fix $h \in H$ and $\varepsilon>0$. There exists $h_{\varepsilon} \in U$ such that $\left|h-h_{\varepsilon}\right|_{H} \leq$ $\varepsilon$. Using (i), we infer that for all $s \in[0, T]$ the following estimates hold

$$
\begin{aligned}
& \left|\left(u_{n} \circ \lambda_{n}(s)-u(s) \mid h\right)_{H}\right| \\
& \leq\left|u_{n} \circ \lambda_{n}(s)-u(s)\right|_{H}\left|h-h_{\varepsilon}\right|_{H}+\left|\left(u_{n} \circ \lambda_{n}(s)-u(s) \mid h_{\varepsilon}\right)_{H}\right| \\
& \leq \varepsilon \cdot\left\|u_{n} \circ \lambda_{n}-u\right\|_{L^{\infty}(0, T ; H)}+\left|\left(u_{n} \circ \lambda_{n}(s)-u(s) \mid h_{\varepsilon}\right)_{H}\right| \\
& \leq 2 \varepsilon \cdot \sup _{n \in \mathbb{N}}\left\|u_{n}\right\|_{L^{\infty}(0, T ; H)}+\left|\left(u_{n} \circ \lambda_{n}(s)-u(s) \mid h_{\varepsilon}\right)_{H}\right| \\
& \leq 2 \varepsilon r+\sup _{s \in[0, T]}\left|\left(u_{n} \circ \lambda_{n}(s)-u(s) \mid h_{\varepsilon}\right)_{H}\right| .
\end{aligned}
$$

Thus

$$
\sup _{s \in[0, T]}\left|\left(u_{n} \circ \lambda_{n}(s)-u(s) \mid h\right)_{H}\right| \leq 2 \varepsilon r+\sup _{s \in[0, T]}\left|\left(u_{n} \circ \lambda_{n}(s)-u(s) \mid h_{\varepsilon}\right)_{H}\right| .
$$

Passing to the upper limit as $n \rightarrow \infty$ we obtain

$$
\limsup _{n \rightarrow \infty} \sup _{s \in[0, T]}\left|\left(u_{n} \circ \lambda_{n}(s)-u(s) \mid h\right)_{H}\right| \leq 2 r \varepsilon .
$$

Since $\varepsilon$ was chosen in an arbitrary way, we get

$$
\lim _{n \rightarrow \infty} \sup _{s \in[0, T]}\left|\left(u_{n} \circ \lambda_{n}(s)-u(s) \mid h\right)\right|=0 .
$$

Since $\mathbb{D}\left([0, T] ; \mathbb{B}_{w}\right)$ is a complete metric space, we infer that $u \in \mathbb{D}\left([0, T] ; \mathbb{B}_{w}\right)$ as well. By Remark 1 this completes the proof of Eq. 96 and of Lemma 2.

\section{References}

1. Adams, R.: Sobolev Spaces. Academic Press, New York (1975)

2. Aldous, D.: Stopping times and tightness. Ann. Probab. t.6 2, 335-340 (1978)

3. Badrikian, A.: Séminaire sur les fonctions aléatoires linéaires et les mesures cylindriques. In: Lect. Notes in Math., vol. 139. Springer, Heidelberg (1970)

4. Billingsley, P.: Convergence of Probability Measures. Wiley, New York (1969)

5. Brezis H.: Analyse Fonctionnelle. Masson (1983)

6. Brzeźniak, Z., Hausenblas, E.: Martingale solutions for stochastic equations of reaction diffusion type driven by Lévy noise or Poisson random measure. Preprint arXiv: 1010.5933v1 [math. PR] 28 Oct (2010)

7. Brzeźniak, Z., Hausenblas, E.: Maximal regularity for stochastic convolution driven by Lévy processes. Probab. Theory Relat. Fields 145, 615-637 (2009)

8. Brzeźniak, Z., Li, Y.: Asymptotic compactness and absorbing sets for $2 \mathrm{D}$ stochastic NavierStokes equations on some unbounded domains. Trans. Am. Math. Soc. 358, 5587-5629 (2006)

9. Brzeźniak, Z., Motyl, E.: Existence of martingale solutions of the stochastic Navier-Stokes equations in unbounded 2D and 3D-domains. Preprint arXiv:1208.3386v1 [math.PR] 16 Aug (2012)

10. Brzeźniak, Z., Ondreját, M.: Stochastic geometric wave equations with values in compact Riemannian homogeneous spaces. Preprint arXiv: 1101.4835v1 [math PR] 25 Jan (2011)

11. Brzeźniak, Z., Peszat, S.: Stochastic two dimensional Euler equations. Ann. Probab. 29(4), 17961832 (2001)

12. Capiński, M., Peszat, S.: On the existence of a solution to stochastic Navier-Stokes equations. Nonlinear Anal. 44, 141-177 (2001)

13. Dong, Z., Xie, Y.: Ergodicity of stochastic 2D Navier-Stokes equations with Lévy noise. J. Differ. Equ. 251, 196-222 (2011) 
14. Dong, Z., Xie, Y., Global solutions of stochastic 2D Navier-Stokes equations with Lévy noise. Sci. China, Ser. A Math. 52(7), 1497-1524 (2009)

15. Dong, Z., Zhai, J.: Martingale solutions and Markov selection of stochastic 3D Navier-Stokes equations with jump. J. Differ. Equ. 250, 2737-2778 (2011)

16. Holly, K., Wiciak, M.: Compactness method applied to an abstract nonlinear parabolic equation. Selected problems of Mathematics, pp. 95-160. Cracow University of Technology (1995)

17. Ikeda, N., Watanabe, S.: Stochastic Differential Equations and Diffusion Processes. NorthHolland Publishing Company, Amsterdam (1981)

18. Jakubowski, A.: The almost sure Skorohod representation for subsequences in nonmetric spaces. Teor. Veroyatnost. i Primenen. 42(1), 209-216 (1997); Translation in Theory Probab. Appl. 42(1), 167-174 (1998)

19. Joffe, A., Métivier, M.: Weak convergence of sequences of semimartingales with applications to multitype branching processes. Adv. Appl. Probab. 18, 20-65 (1986)

20. Lions, J.L.: Quelques Méthodes de Résolution des Problèmes Aux Limites Non Linéaires. Dunod, Paris (1969)

21. Métivier, M.: Semimartingales. Gruyter (1982)

22. Métivier, M.: Stochastic partial differential equations in infinite dimensional spaces. Scuola Normale Superiore, Pisa (1988)

23. Métivier, M., Viot, M.: On weak solutions of stochastic partial differential equations. Lect. Notes Math. 1322/1988, 139-150 (1988)

24. Mikulevicius, R., Rozovskii, B.L.: Global $L_{2}$-solutions of stochastic Navier-Stokes equations. Ann. Probab. 33(1), 137-176 (2005)

25. Motyl, E.: Martingale Solutions to the 2D and 3D Stochastic Navier-Stokes Equations Driven by the Compensated Poisson Random Measure, Preprint 13. Department of Mathematics and Computer Sciences, Lodz University (2011)

26. Peszat, S., Zabczyk, J.: Stochastic Partial Differential Equations with Lévy Noise. Cambridge University Press, Cambridge, UK (2007)

27. Temam, R.: Navier-Stokes Equations. Theory and Numerical Analysis. North Holland Publishing Company, Amsterdam - New York - Oxford (1979)

28. Vishik, M.J., Fursikov, A.V.: Mathematical Problems of Statistical Hydromechanics, Kluwer Academic Publishers, Dordrecht (1988)

29. Xu, T., Zhang, T.: Large deviation principle for 2-D stochastic Navier-Stokes equations driven by Lévy processes. J. Funct. Anal. 257, 1519-1545 (2009) 